\title{
The Public Health Impact of
}

\section{Minor Injury}

Roderick McClure

A thesis submitted for the degree of Doctor of Philosophy of the Australian National University, August 1994 
Except where otherwise indicated, this thesis is entirely my own original work.

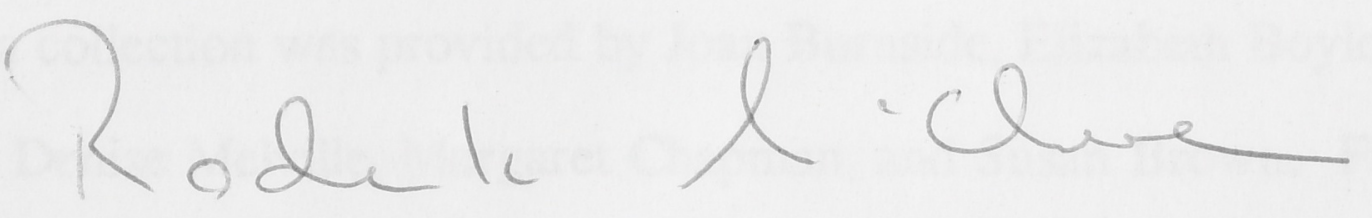




\section{Acknowledgments}

The ideas for this thesis were developed while I was on a World Health Organization Travelling Fellowship in 1991 and I am grateful to the WHO for their sponsorship. Assistance with data collection was provided by Joan Burnside, Elizabeth Boyle, Jenny Chadwick-Masters, Denise Melville, Margaret Chapman, and Susan Brown. Financial assistance for expenses relating to the data collection was provided by ACT Health, the National Centre for Epidemiology and Population Health, and the Commonwealth Department of Health Housing and Community Services. The research was conducted while I was receiving an Australian National University Scholarship.

I am grateful to the medical, nursing and clerical staff of the general practices and emergency departments in the ACT who collected data for use in the thesis and to the patients involved in the study.

Thanks to Lin Fritschi and David Crawford for your support and for teaching me some epidemiology. I would also like to thank Lin Fritschi, Bruce Armstrong, Len Smith, and Bob Douglas for reading and commenting on earlier drafts of this thesis.

Finally, I would like to acknowledge the helpful advice on matters of study design and analysis provided by Gavin Andrews, Robyn Attewell, Erich Kliewer, Gigi Santow, Aileen Plant, Joan Ozanne-Smith, James Harrison, Bruce Shadbolt and Rosemary Knight. 


\section{Abstract}

This thesis is the report of a research programme undertaken to evaluate the public health impact of non-life-threatening injuries.

Incidence and distribution of injury

A population-based incidence survey was conducted using random and systematic samples in a region with known demographic characteristics. The injury rate in this population was 164 per 1,000 person years $(95 \% \mathrm{CI}=139-189)$ and the ratio of deaths : hospital admissions : emergency department attendances : general practice treatments was 1:28:596:592.

\section{Health cost of injury}

An injury outcome instrument, the Health Consequences of Injury Questionnaire (HCIQ), suitable for use in large scale epidemiological surveys was developed and validated in a sample of injured adults. A sample of adults was drawn from the Australian Capital Territory Injury Surveillance and Prevention Project database (previously evaluated in terms of reliability and sensitivity) and followed for a period of six months using the HCIQ. It was shown that $90 \%$ of the morbidity arising from injury is due to those injuries that do not require immediate hospitalisation at the time of their first presentation for treatment.

\section{Economic cost of injury}

An incidence-based estimate was made of the economic cost of managing acute nonlife-threatening injuries in the study population. This included establishing the cost of treating non-life-threatening injuries in hospital emergency departments as well as the cost of treating acute injuries in General Practice. The cost of this service provision was found to impose a substantial burden on the health expenditure of the community.

\section{Minor Injury Scale}

The Minor Injury Scale was developed from data obtained in the health cost of injury study. The injury scoring system enables non-life-threatening injuries to be categorised and ranked on the basis of their potential for resulting in adverse health outcomes.

Conclusion: This thesis shows that non-life-threatening injuries constitute the vast majority of all injuries, that non-life-threatening injuries are responsible for the major part of the morbidity that arises from injury, and that non-life-threatening injuries result in substantial economic costs. Non-life-threatening injuries need to be recognised as a community health problem of major importance. Considerable public health gains can be made by directing injury prevention efforts not towards those injuries that carry the greatest cost to the individual, but towards those groups of injuries responsible for the greatest cost to the population. 


\section{List of publications}

Three papers based upon work reported in this thesis have been accepted for publication.

McClure RJ, Burnside J. The Australian Capital Territory Injury Surveillance and Prevention Project. Academic Emergency Medicine (in press).

McClure RJ. The Health Consequences of Injury Questionnaire. Academic Emergency Medicine (in press).

McClure RJ. The Minor Injury Severity Score. Academic Emergency Medicine (in press). 


\section{Contents}

Contents

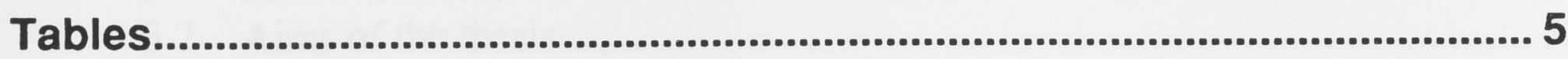

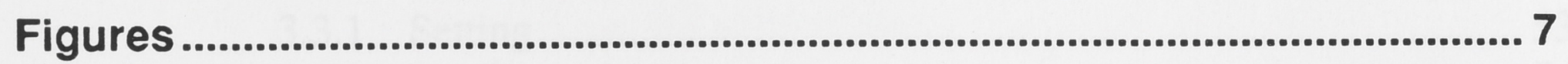

\section{Chapter One}

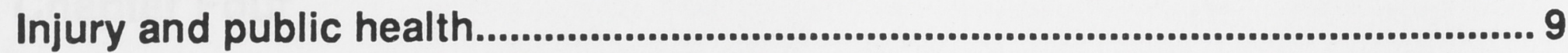

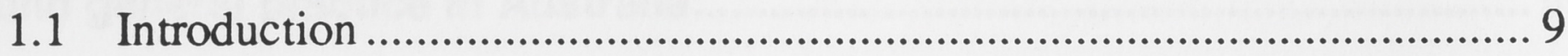

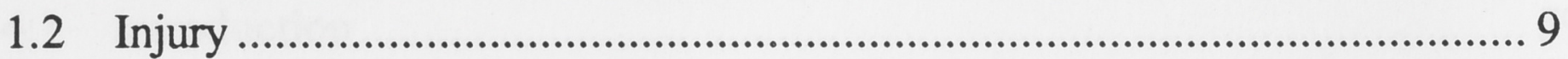

1.2.1 Injury severity scales .............................................. 10

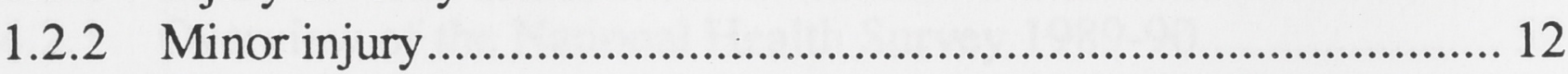

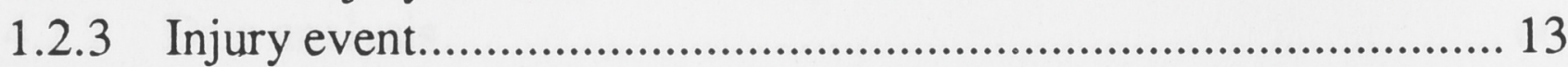

1.3 Injury as a public health problem ............................................... 14

1.4 The historical development of the public health approach...................... 15

1.5 Extending the public health model of injury ................................... 17

1.5.1 The current conceptualisation of health ................................ 17

1.5.2 The population as the focus of public health ......................... 19

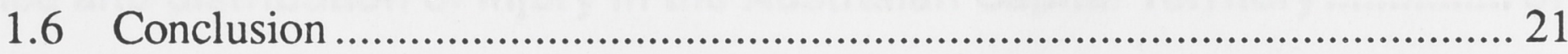

\section{Chapter Two}

Review of current knowledge of the public health impact of injury .................... 22

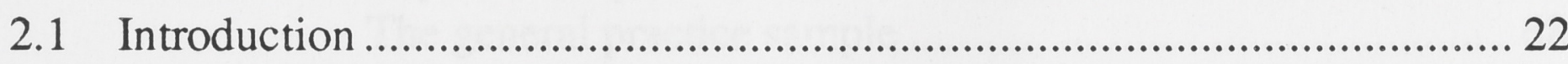

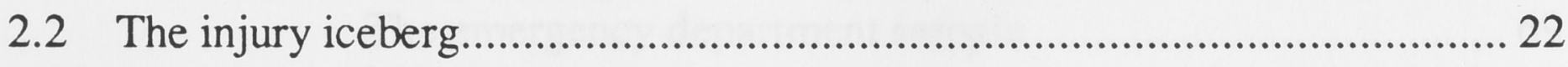

2.3 Incidence and distribution of injury ............................................ 24

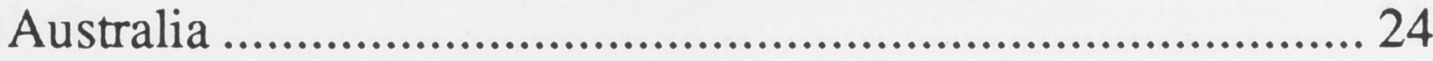

i) Sources of information ....................................... 24

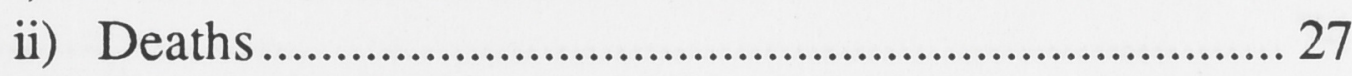

iii) Hospital inpatient care ....................................... 27

iv) Emergency department cases.............................. 27

v) General practice cases........................................ 28

vi) Total picture estimates........................................ 29

United States of America .............................................. 31

Rest of the world ....................................................... 33

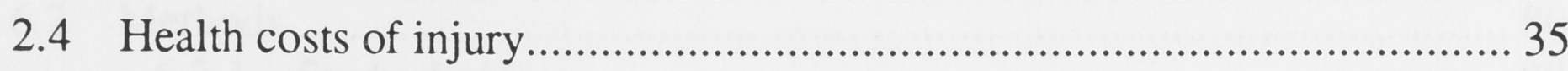

2.4.1 Outcome methodology ............................................... 35

2.4.2 Outcomes of injury ........................................................... 41

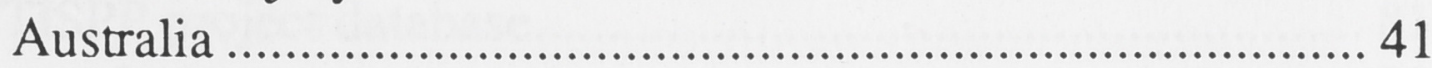

United States of America .............................................. 42

Rest of the world .......................................................... 43

2.4.3 Predicting outcomes.................................................. 45

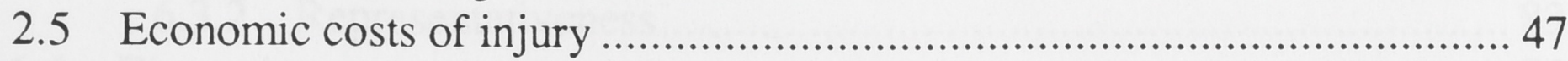

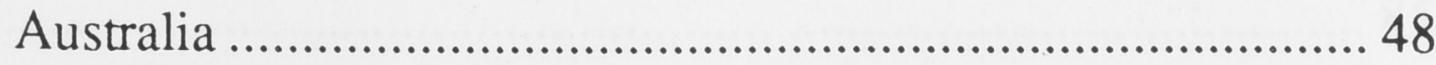

United States of America .............................................. 49

Rest of the world ....................................................... 49 


\section{Chapter Three}

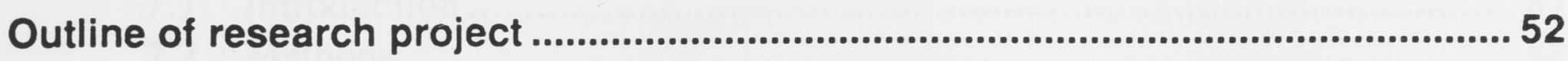

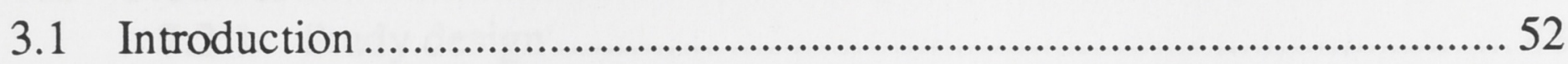

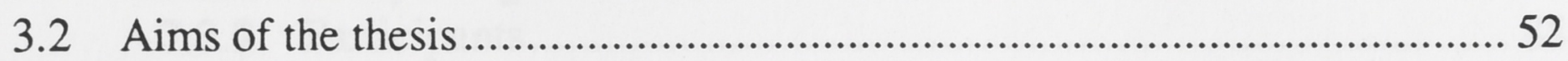

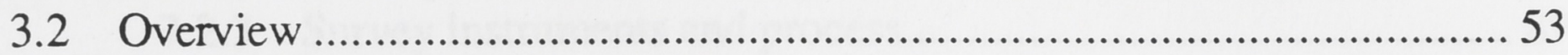

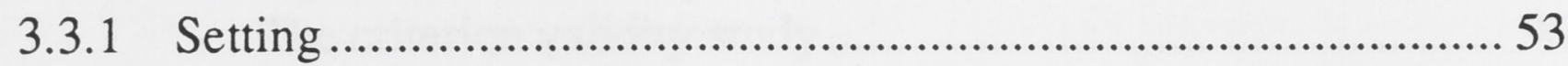

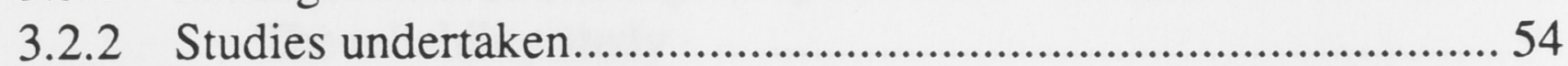

\section{Chapter Four}

Injury and general practice in Australia............................................................... 59

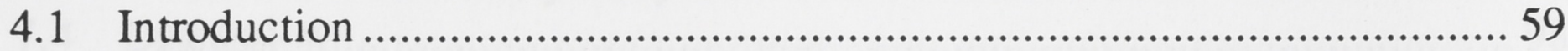

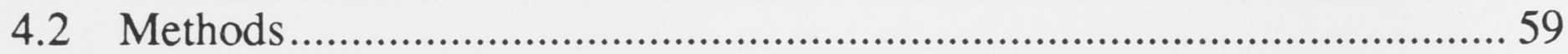

4.2.1 Overview of the National Health Survey 1989-90_.................... 59

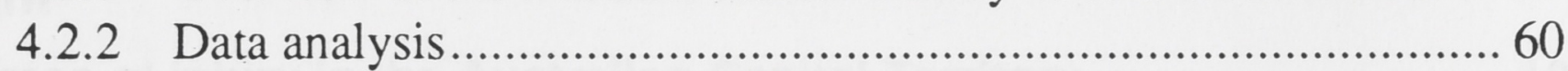

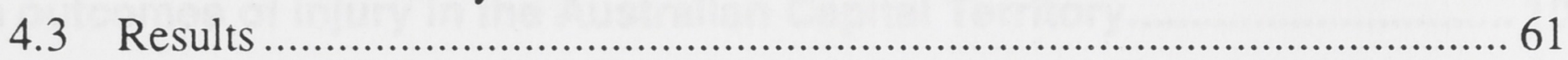

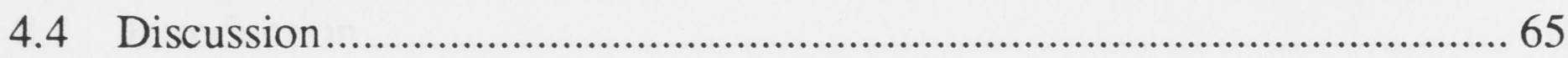

\section{Chapter Five}

Incidence and distribution of injury in the Australian Capital Territory................67

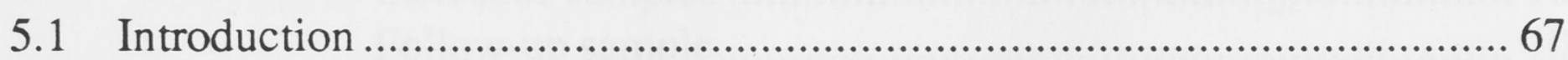

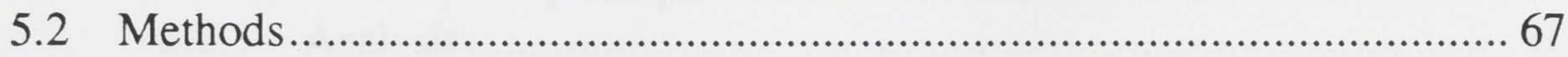

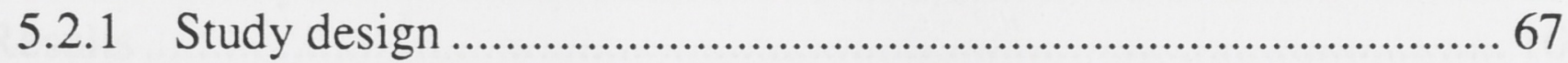

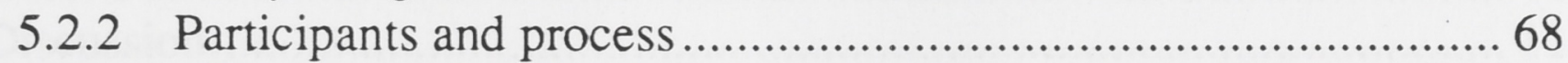

The general practice sample ……………………………......6 68

The emergency department sample .......................................69 69

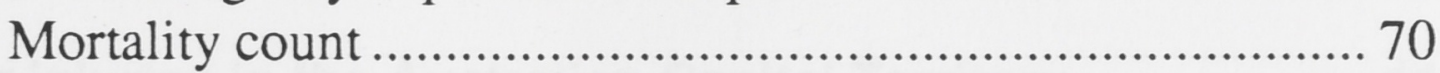

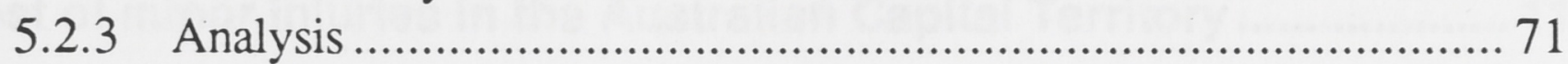

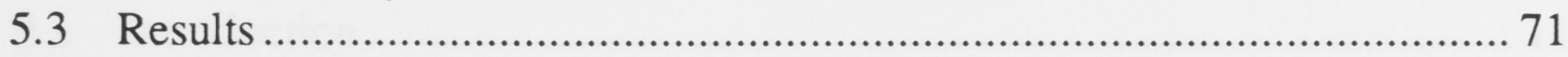

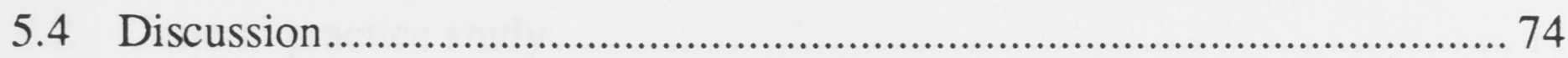

\section{Chapter Six}

The Australian Capital Territory Injury Surveillance and Prevention

Project 80

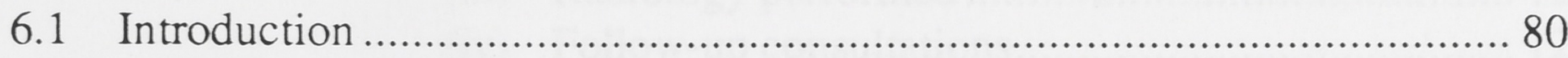

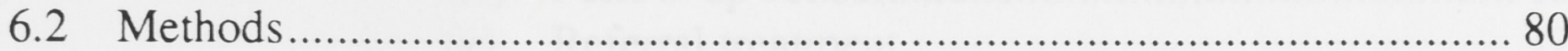

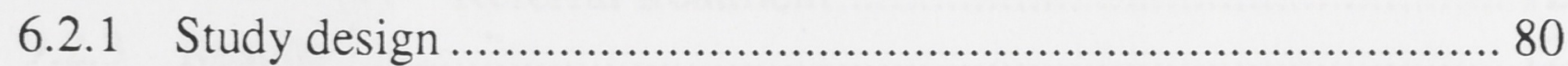

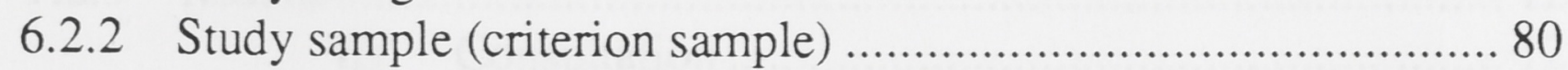

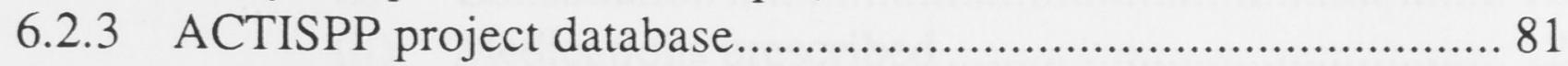

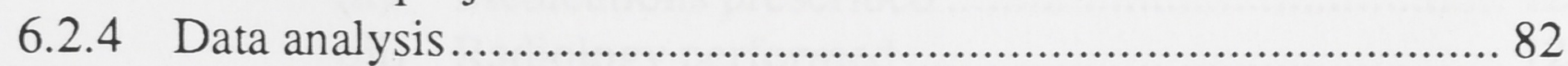

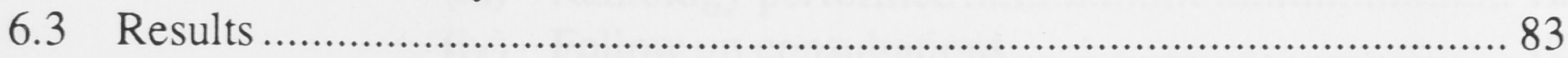

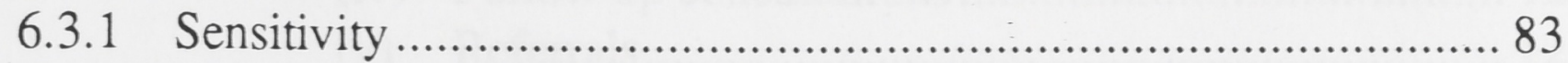

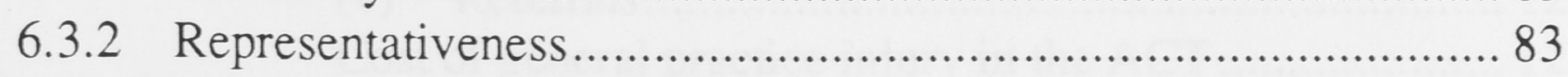

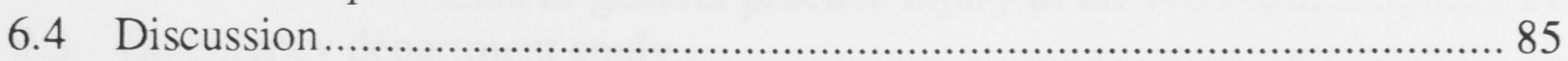


Chapter Seven

The Health Consequences of Injury Questionnaire 87

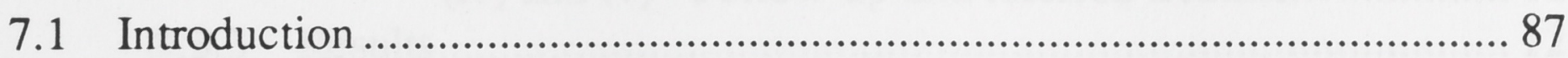

7.2 Methods..................................................................... 88

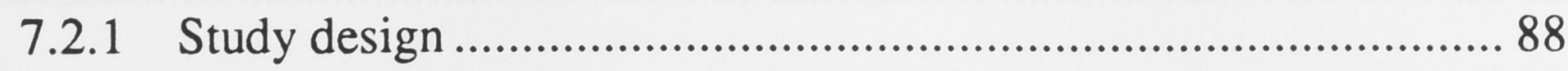

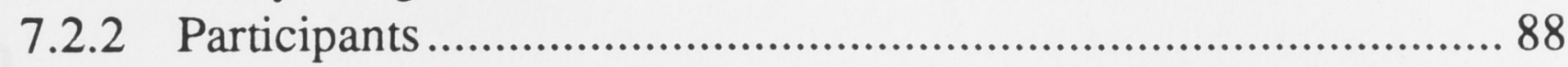

7.2.3 Survey instruments and process.............................................. 89

The criterion validity study ................................................. 90

The reliability study …………………………………........ 91

7.2.4 Measurement and data analysis ………………...................... 91

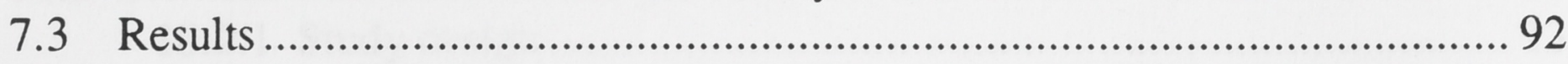

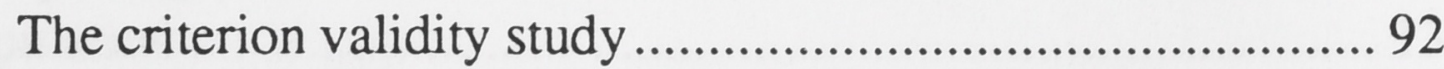

Reliability study .............................................................. 96

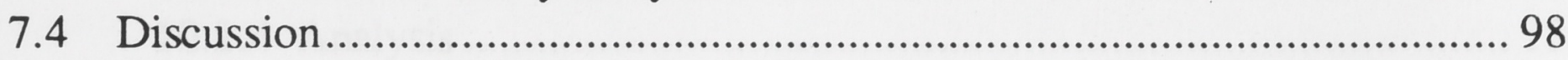

\section{Chapter Eight}

Health outcomes of injury in the Australian Capital Territory............................... 102

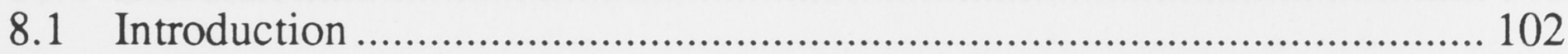

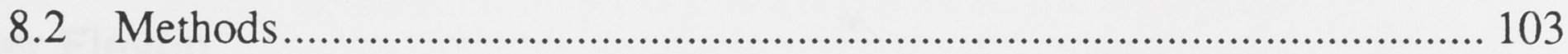

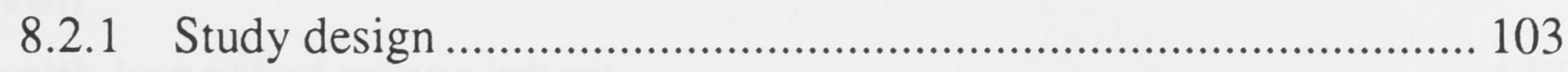

8.2.2 Participants and process ........................................................... 103

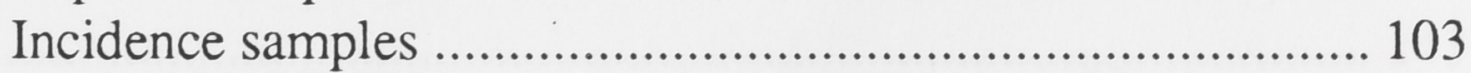

Follow-up sample............................................................. 103

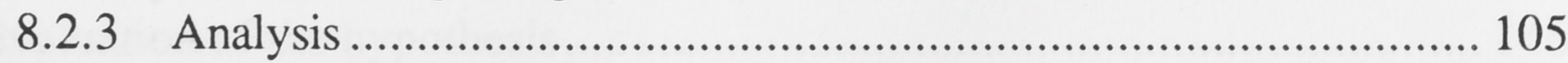

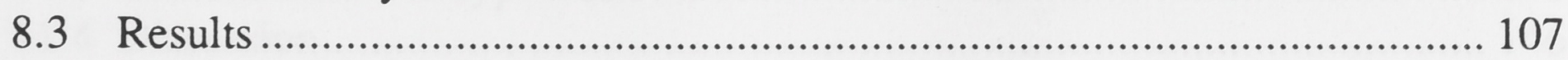

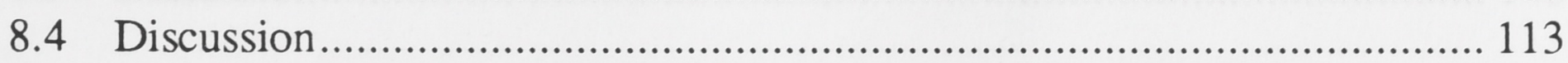

\section{Chapter Nine}

Economic cost of minor injuries in the Australian Capital Territory ..................... 116

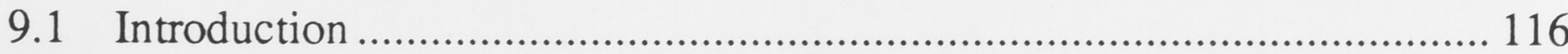

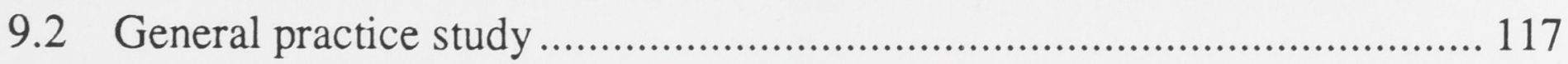

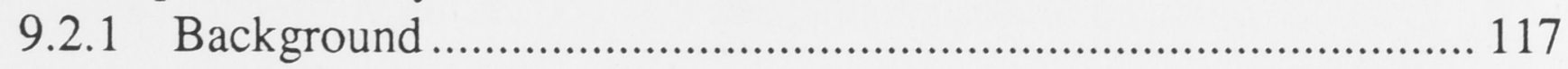

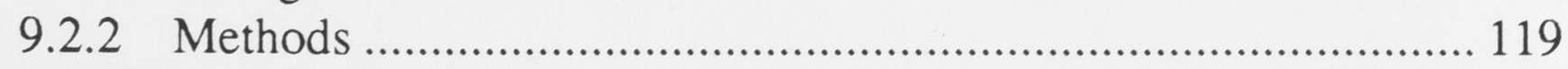

(i) Consultation ................................................... 120

(ii) Medications prescribed ....................................... 121

(iii) Radiology performed ........................................... 121

(iv) Follow-up consultations........................................ 122

(v) Referral treatment........................................... 122

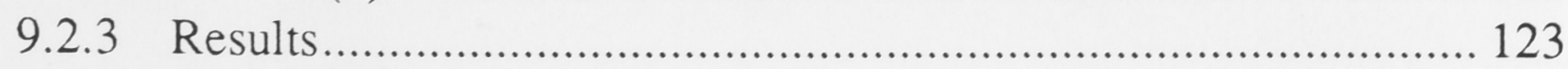

(i) Consultation ……............................................ 123

(ii) Medications prescribed ........................................ 124

(iii) Radiology performed .......................................... 124

(iv) Follow up consultations ....................................... 125

(v) Referrals............................................................ 125

Cost of general practice injury in the ACT ..................... 125

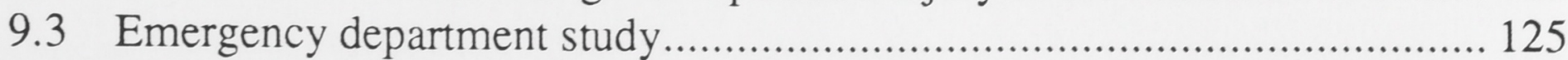

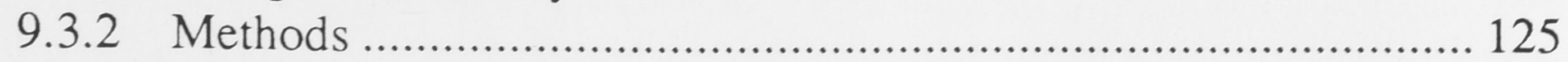

(i) Consultation and treatments.................................. 127 
(ii) Radiology ordered ........................................... 127

(iii) Medications prescribed .................................... 128

(iv) and (v) Follow-up and referred treatment ............. 128

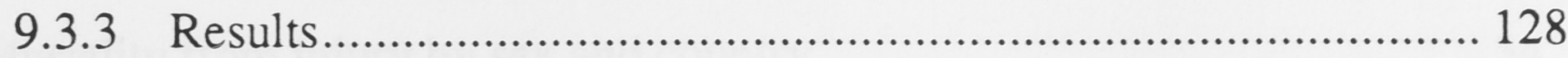

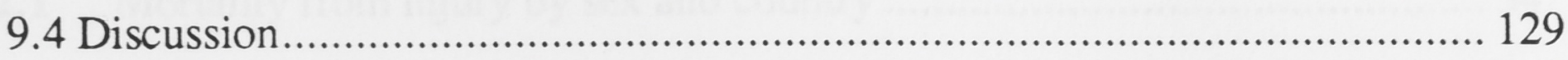

\section{Chapter Ten}

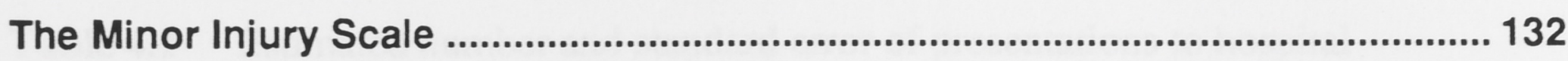

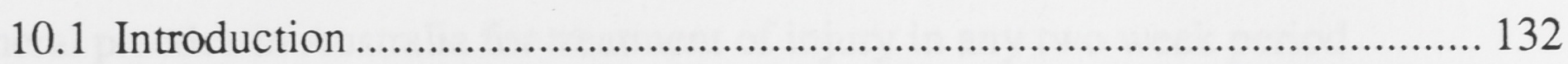

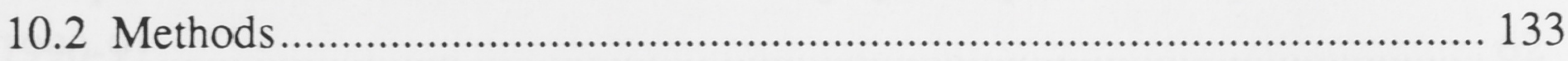

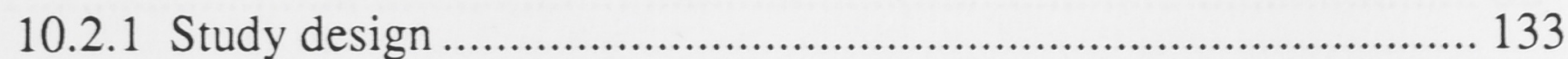

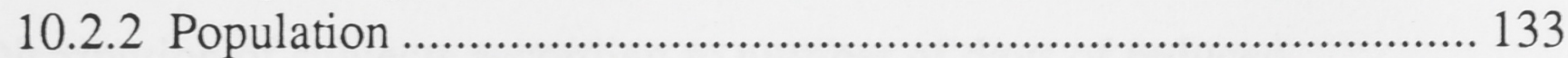

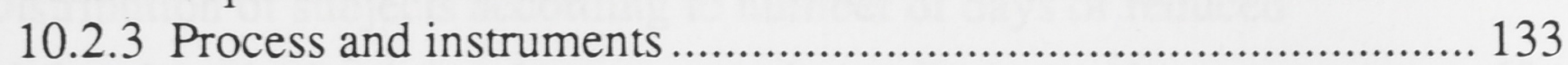

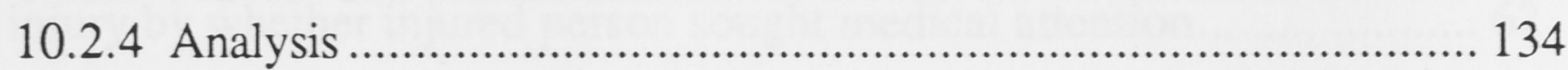

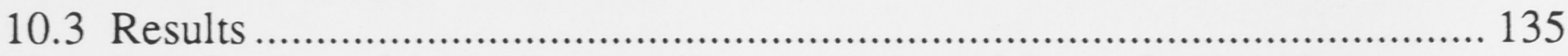

10.3.1 Reporting bias and variable distribution ................................ 135

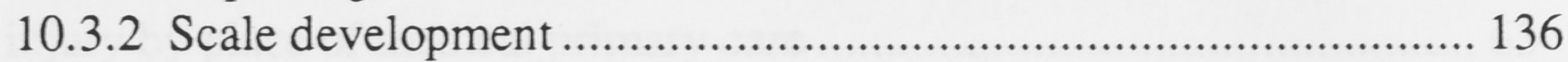

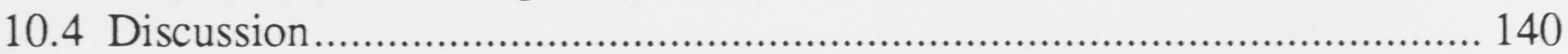

\section{Chapter Eleven}

The public health impact of minor injury ............................................................. 143

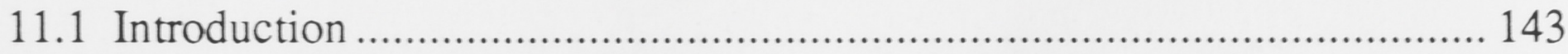

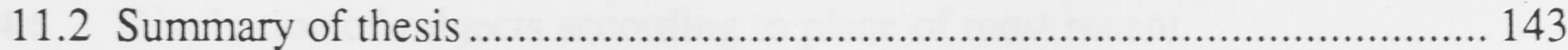

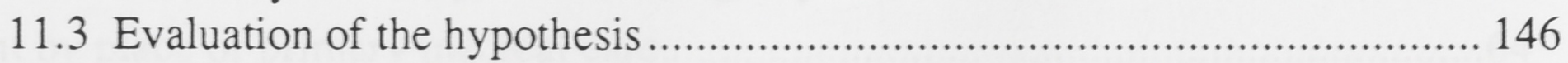

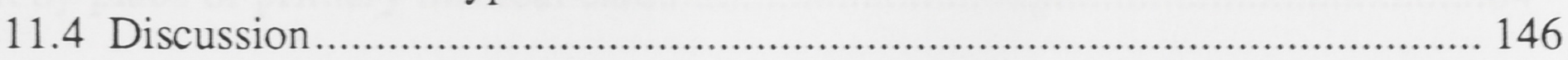

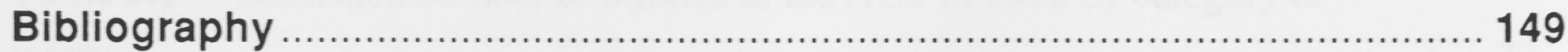

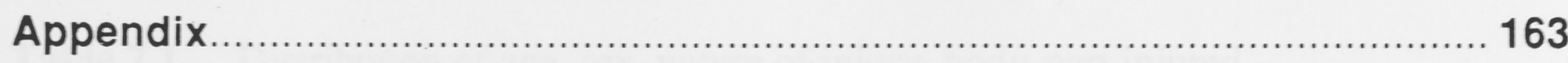




\section{Tables}

Table 2.1 Mortality from injury by sex and country ..................................... 34

Table 3.1 Summary of the research reported in this thesis 58

Table 4.1 Estimated number and standard error of presentations to hospital and general practice in Australia for treatment of injury in any two week period during 1990-91.

Table 4.2 Distribution of subjects according to number of days of reduced activity due to injury by whether injured person sought medical attention.

Table 4.3 Distribution of subjects according to number of days of reduced activity due to injury by place of medical primary care

Table 4.4 Logistic regression analysis of characteristics which distinguish the subjects who presented to general practice rather than an emergency department for treatment of an injury during the two weeks prior to interview.

Table 4.5 Disribution of subjects according to place of most recent accident by place of primary medical care

Table 5.1 Estimated number of injuries in the ACT in 1992 by category of injury.

Table 7.1 Distribution of age, sex, nature of injury, body-part injured, Abbreviated Injury Scale (AIS) severity scores, context of injury, and admission status for the criterion-validity-study responders and non-responders.

Table 7.2 The mean Health Consequences of Injury Questionnaire (HCIQ) estimated score and standard deviations and number of occurrences by injury type for those injuries occurring on more than one occasion in the sample.

Table 7.3 Regression of Quality of Well-Being score on HCIQ estimates 95

Table 7.4 Distribution of age, sex, nature of injury, body-part injured, AIS severity scores, context of injury, and admission status for the reliability-study responders and non-responders.....

Table 8.1 Distribution of age, sex, nature of injury, body part injured and whether hospitalised in the incidence and follow-up samples. 108 
Table 8.2 Estimated total quality adjusted life-years (QALYs) lost in the Australian Capital Territory due to injury in adults during 1992 by outcome category

Table 8.3 Estimated QALYs lost in the Australian Capital Territory due to non-fatal injury in adults during 1992 by nature of injury, stratified by age and $\operatorname{sex}$

Table 8.4 Estimated QALYs lost in the Australian Capital Territory due to injury in adults during 1992 by body-part injured, stratified by age and sex.

Table 8.5 Estimated QALYs lost in the Australian Capital Territory due to injury in adults during 1992 by (AIS) severity score

Table 9.1 Distribution of incidence sample and radiology samples by sex, injury type and body-part injured

Table 10.1 Distribution of samples by age, sex, nature of injury, body-part injured and whether admitted

Table 10.2 Extract from the Minor Injury Scale listing the Minor Injury Severity Score and the Minor Injury Morbidity Score for six injury types identified by AIS codes.

Table 10.3 Minor Injury Scale. 


\section{Figures}

Figure 1.1 Schematic representation of the epidemiological model of injury. 16

Figure 2.1 The injury iceberg.

Figure 5.1 Injury rate in the Australian Capital Territory (ACT) by age and sex.

Figure 5.2 Distribution of injuries in the ACT by place of treatment and body-part injured

Figure 5.3 Distribution of injury in the ACT by place of treatment and nature of injury.

Figure 6.1 Distribution of age in ACT Injury Surveillance and Prevention Project (ACTISPP) database and criterion sample

Figure 6.2 Distribution of postcode of residence for the ACTISPP database and criterion sample.

Figure 6.3 Distribution of nature of injury for the ACTISPP database and criterion sample 84

Figure 6.4 Distribution of body-part injured for ACTISPP database and criterion sample

Figure 7.1 Scattergram of Health Consequences of Injury Questionnaire (HCIQ) estimates and Quality of Well-Being score 95

Figure 7.2 Scattergram of HCIQ estimates obtained from test-retest study 96

Figure 8.1 Graph of health loss against time for a hypothetical injury which occurred at time ' 0 ' and recovered at time ' $t$ ' to illustrate definition of injury morbidity.

Figure 8.2 Cumulative proportion of follow-up sample unrecovered over time plotted with linear-exponential approximations

Figure 9.1 Incidence of injuries presenting to general practice as place of first treatment by age and sex.

Figure 11.1 The injury iceberg: percent of total medically-treated injuries in the ACT during 1992 by category. 
Figure 11.2 The injury iceberg: percent of total Quality Adjusted Life Years lost from medically-treated injuries in the ACT during 1992 by category..... 


\section{Chapter One}

\section{Injury and public health}

\subsection{Introduction}

Injury has been identified by the Australian Health Ministers as one of the four focus areas of major public health importance in Australia. National goals and targets for the improvement of injury-related health outcomes are embedded in National Health Policy (Health Ministers' Forum 1994; AHMAC/NH\&MRC working groups 1994b).

\subsection{Injury}

Injury is defined as:

unintentional or intentional damage to the body resulting from acute exposure to thermal, mechanical, electrical, or chemical energy or from the absence of such essentials as heat or oxygen (National Committee for Injury Prevention and Control 1989:4).

This aetiological definition of injury has wide acceptance (Committee on Trauma Research 1985:3-4; Better Health Commission 1986:197). However, it is not in a form that is convenient for use as a case definition in epidemiological studies. Hence, injury is often defined as any condition classifiable according to ICD-9 codes 800-995 (World Health Organization 1977; Fife et al. 1984; Rivara et al. 1989). The advantage of the ICD-9 system is that it can be used to identify injury by nature of injury and body-part injured as well as by external cause of injury (E code).

A disadvantage of the ICD-9 definition of injury is that it does not contain any reference to injury severity; yet specification of severity is clearly important:

At the minor end of the spectrum where does injury start? ..... Injury occurs when the intensity of energy transferred exceeds the tolerance of the body..... This threshold is not well defined (Harrison 1991:15).

In practice, studies on injury usually deal with only those injuries sufficiently serious to require medical attention (Sahlin et al. 1990; Lodge et al. 1990). 
In accordance with accepted practice, the definition of injury used for the research described in this thesis is the aetiological definition presented above. The case definition is:

any condition that can be classified according to ICD-9 codes 800 to 995 and that registers for medical attention.

\subsubsection{Injury severity scales}

The specification of injury severity is essential not only to the definition of injury but also to the use of injury epidemiology for injury control (Robertson 1992:29). Injury is a global term to describe an extensive range of conditions covering a variety of types and severities. Epidemiological study of these injuries is not possible without a means to group them into relevant subsets on the basis of severity. Injury severity scales provide the means by which these groupings can be made.

As has been noted above, the ICD-9 classification system does not rank injury by severity although it has been suggested that some idea of injury severity can be inferred (Rutledge et al. 1993). The Abbreviated Injury Scale (AIS) is another injury classification that is commonly used (Baker et al. 1974; Committee on Injury Scaling 1990). This system categorises injury in greater detail than the ICD-9 and includes a severity score. The AIS severity score ranks injuries according to their threat-to-life (States and Viano 1990).

Several other severity scores have been developed for specific purposes, mostly for aiding trauma management or identifying potentially fatal injuries. Examples of these include the Paediatric Trauma Score (PTS) (Tepas et al. 1988), A Severity Characterisation of Trauma (ASCOT) (Champion et al. 1990), and the Probability of Death Score (Sommers 1983). In contrast to the numerous and well evaluated major trauma scores (Gustafson et al. 1983; Teijink et al. 1993), scales for categorising nonlife-threatening injuries are few and poorly validated. 
The need for a non-fatal-injury outcome scale has been recognised since the early 1980s. The most complete of the early attempts was published in 1990 (States and Viano 1990). Using the WHO definition of impairment and disability, the authors developed an ordinal, seven point impairment scale with zero representing no impairment and six representing uniformly total impairment. Given injuries could be scored on this scale for each of the dimensions of impairment: mobility/dexterity; cognitive; social; cosmetic/disfigurement; sensory; pain; reproduction. Whole body impairment was taken as the maximum impairment for any body region.

In 1994, the Association for the Advancement of Automotive Medicine proposed a measure of impairment based upon a variation of the WHO definition (Committee on Injury Scaling 1994) called the Injury Impairment Scale (IIS). The IIS scored predicted loss of function at twelve months after injury and used 'impairment' to refer to whole body dysfunction rather than organ or system dysfunction. To each of the numerical injury descriptions from the AIS is attached a number from 0 to 6 indicating the level of impairment that might be expected to occur in at least $80 \%$ of survivors. This set of impairment value weights was assigned by a panel of experts and has not yet been validated.

Perhaps the most sophisticated of the injury scales for scoring the outcome of non-fatal injuries currently under development is the Functional Capacity Index (National Highway Safety Administration 1992). It is planned that this scale will rate each injury in the AIS in terms of a factor that can be applied to the patient's remaining years of life to produce an adjusted life-year prediction of consequent functional incapacity.

Several other attempts at developing a scale for non-fatal injury have been reported. In 1991, a system was trialed for measuring both temporary and permanent disability arising from injury (Yates et al. 1991). The investigators followed a sample of 500 injured patients at one and six months after injury and assessed their level of disability by means of a questionnaire. They were unable to construct a definitive scale because 
of small numbers and concerns about the ability of the instruments used to identify disability in their sample.

In 1992, an attempt at devising a disability score for non-hospitalised patients was made using empirical data from a follow up study (Alexander et al. 1992). The outcome measure used for this study was a physical disability instrument from the Rand Insurance Study which was administered at one week and one month after the injury occurrence. The authors in this study concluded that they were unable to arrive at an injury disability score and that other outcome instruments or scale development techniques would be necessary.

Injury severity scales for categorising major injury on the basis of 'likelihood of death' are well developed and widely used. There are currently no injury scales suitable for categorising non-life-threatening injury on the basis of predicted health outcomes.

\subsubsection{Minor injury}

Historically, one of the central uses of injury severity scales has been to distinguish between major and minor injury. The importance of this distinction is explained by Robertson (1992:30):

Since trivial injuries are so common, priority in the devotion of resources to control of injuries based on total numbers in a given category can result in a substantial misallocation of resources with respect to reducing the cost of injuries and the improvement of the quality of life of the severely injured or their families.

The implication here is that resources should be allocated, not to the prevention of minor injuries "which heal with little or no treatment" (Robertson 1992:30), but to the control of severe or major injuries. This view has wide support. In Australia, for example, it has been suggested that:

Efforts to prevent.........accidents should be directed at the causes of serious injury and death, rather than at the causes of the vast number of emergency department trivial injuries (Hurst and Ellis 1977:768) 
and that:

In particular, attention should be focused on deaths from transport accidents and drowning (O'Connor 1983:230).

Further demonstration that this view is the current orthodox position can be found in the current Australian public health strategy for injury control (Nutbeam et al. 1993) which almost exclusively identifies deaths, serious injuries and hospitalised injuries as the focus of national efforts.

Although there is a clear consensus in the literature concerning the general use of the term 'minor injury', there is some variation in the actual definition. In the AIS, 'minor' injuries are those which constitute the least threat to life and include superficial abrasions, lacerations, bruising, sprains, first degree burns and some fractures. In a recent Australian study of injury severity scoring, 'minor injuries' were defined as those injuries:

associated with a low risk of requiring admission to hospital (Collopy et al. 1992:489).

In this thesis, the term 'minor injury' is reserved for those injuries which pose no real threat to life. As life-threatening injuries that present for medical assessment in Australia are invariably admitted to hospital, minor injuries were identified with those injuries which were not admitted to hospital at the time of their first presentation for medical assessment.

\subsubsection{Injury event}

Much of the work in the field of injury control focuses not on the injury itself but upon the event that produces the injury. For example, the aim of a recent study was "to survey all accidents occurring in a defined population" (Sahlin et al. 1990:155). In this study 'accident' was defined as "a sudden external occurrence leading to personal injury". 
Elsewhere accidents have been defined as:

sudden series of undesirable occurrences in interplay between individual and environment which have led to personal injury (Schelp and Svanstrom 1987:92)

and:

sudden, unanticipated, random events that cause unwanted damage (Waller 1987:27).

However, as early as 1961, Suchman argued that the understanding of the term 'accident' was inhibiting the advancement of injury control. To continue to use the term accident is to perpetuate the popular notion that accidents are not subject to the usual cause and effect relationships that allow public health prevention processes to be effective. This position has been accepted by injury epidemiologists (Waller 1987:27; Langley 1988) although the term 'accident' persists in some areas for historical reasons.

\subsection{Injury as a public health problem}

Since injury damages the organism and overwhelms the normal physiological homeostatic mechanisms, it clearly compromises a person's health. Once injury is described in terms of its aetiology, the distinctions between injury and disease disappear (Haddon 1980b:414; Waller 1987:27; Langley 1988) and the theory and practices of public health can quite properly be applied to the field of injury control.

Last (1988:107) defines public health as one of the efforts organised by the society to protect, promote and restore the health of a population. A condition becomes of public health concern when it has a substantial effect on the health of a substantial number of people in a community. Chapter Two presents a review of current knowledge of the health effects of injury throughout the world. This overview of injury-related mortality and morbidity clearly establishes injury as a public health concern for the international community. 


\subsection{The historical development of the public health approach}

Despite the existence of injury in the community since earliest times and its devastating effect on the health of populations (Haddon 1980b:411), attempts to deal with injury as a public health problem are relatively recent phenomena. Waller (1989:272) argues that the first overt acknowledgment that injury and its prevention was the responsibility of national public health programmes probably came in 1779 with the publication of Medicinische Polizey, a public health tract by Johann Frank (Baumgartner and Ramsey 1933). Waller suggests that the next real progress in this area was not made until the 1950s when, in the USA, developing State Departments of Health initiated widespread injury prevention programmes. The nature of these early efforts reflected the contemporary conceptualisations of the injury problem. In the belief that injury was predominantly a product of carelessness and clumsiness, the control strategies focussed on education and behaviour change in individuals (Chapman 1961:vi). These early prevention strategies were neither data-driven nor evaluated.

At about this time, child injury prevention workers recognised the importance of reducing hazards in the environment and pioneered the team approach to injury control (American Public Health Association 1956). Traffic safety workers developed this environmental management and interdisciplinary approach during the 1950 s and 60s to sophisticated levels (National Committee for Injury Prevention and Control 1989:121). Drawing on expertise from the disciplines of engineering, biophysics, medicine and public safety, and based upon sound research and interdisciplinary cooperation, the traffic safety strategy served as a model soon adopted by public health officials.

The public health model was characterised by an environmental approach (De Haven 1942), the sound use of data and the application to injury of epidemiological principles initially developed for infectious disease (Gordon 1949). Gibson, (1961), further elucidated the parallel mechanisms of injury and disease and Haddon developed his influential strategies for injury control based upon these epidemiological principles (Haddon 1963; Haddon et al. 1964). The 'epidemiological model' was thus established 
as the central tenet of injury control. A schematic explanation of the epidemiological model is reproduced in Figure 1. The subsequent work of leading injury control practitioners continues to affirm the value of this model for facilitating the prevention of injury (Haddon 1980; Waller 1985; Schelp 1987; Robertson 1992:8).

Figure 1.1 Schematic representation of the epidemiological model of injury.

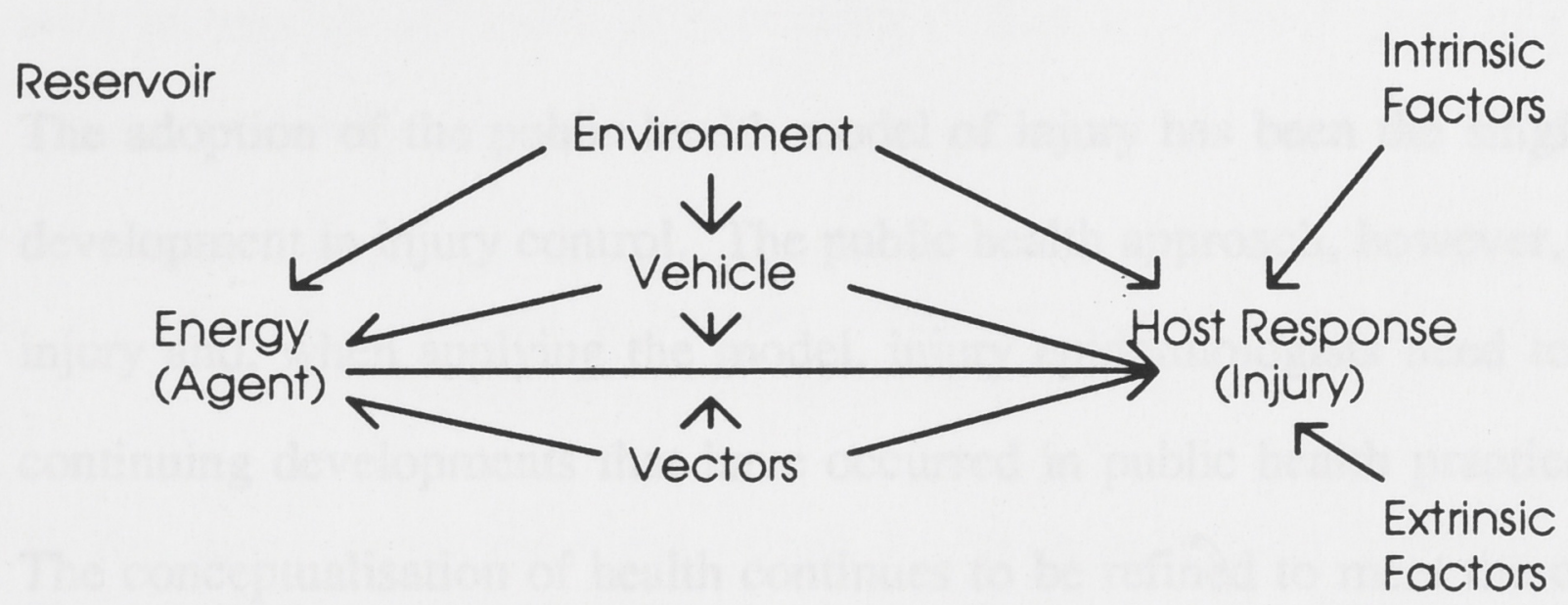

(Kraus and Robertson 1992:1022)

Injury control today has been described as the continuing and broadening of Haddon's concepts with far more sophisticated use of behavioural interventions. The current approach involves:

research and data collection, professional training, well-conceived health department programs, and coordination at both federal and local levels between public health workers and others with legitimate concerns in this field (Waller 1989:272).

Progress in injury control in Australia in the 1970s followed closely the public health developments of the United States. In 1970 it was observed that:

the commonsense approach (to injury control) and its associated folk activity can now be described as palpably ineffective and due for replacement (Wigglesworth 1970:1114).

Between 1970 and 1989, published articles outlined the informational requirements for effective injury prevention (Wigglesworth 1979; Vimpani et al. 1989) and demonstrated the use of epidemiology as a strategy for injury control (Jamieson and 
Wigglesworth 1977). By 1989, the advantages of the epidemiological approach were clearly apparent (Wigglesworth 1989; Vimpani 1989) and it could be claimed that:

Scientific and systematic approaches to injury control which rely on changing the environment or injury-causing agent rather than pleas for sensible behaviour are now becoming increasingly common (Vimpani 1989:10).

\subsection{Extending the public health model of injury}

The adoption of the public health model of injury has been the single most important development in injury control. The public health approach, however, is not peculiar to injury and, when applying the model, injury epidemiologists need to be aware of the continuing developments that have occurred in public health practice in recent years. The conceptualisation of health continues to be refined to meet the changing needs of the community. Prevention strategies have become more sophisticated and health measures have been devised that better assess the theoretical constructs being measured. The public health approach to injury control has been evident for over thirty years, yet injury remains one of the key causes of morbidity in all communities of the world. The philosophy and practice of injury control must continue to be refined if it is to maintain its efficacy in contemporary societies.

Two changes of emphasis need to be made in the current injury model to keep it consistent with contemporary concepts of public health. The first of these relates to the definition of health (Section 1.5.1). The second concerns the purpose of public health programmes (Section 1.5.2).

\subsubsection{The current conceptualisation of health}

In the thirty years since the public health model of injury first became widely accepted, the definition of health has undergone continual change (Minaire 1992). Initially health was understood in terms of 'survival'. It later became recognised as 'freedom from disease' and then 'an individuals ability to perform his daily activities'. Health is now 
defined in terms of the positive themes of 'well-being' and 'quality of life'. This contemporary understanding of health is reflected in the constitution of the World Health Organization where health is described as:

a state of complete physical, mental and social well-being and not merely the absence of disease or infirmity (World Health Organization 1984:1).

The contemporary understanding of health is also reflected in statements from Australian public health physicians, who acknowledge that public health is "concerned with promotion of health in populations" (Leeder 1991:7) and in current Australian health policy which is based on the assumption that:

measurement of health outcomes goes beyond mortality and morbidity to take into account a broader measure of well-being and quality of life (AHMAC/NH\&MRC working groups, 1994a:19).

In the thirty years since the public health approach to injury control was widely adopted there have also been changes in the concept of injury-related health. These changes have followed the changes in the general health model but lag several years behind. Initially, injury was recognised in terms of fatalities and successful prevention was measured in terms of lives saved. As recently as 1984 the culture of injury prevention was still equating injury with injury fatalities. The Injury Fact Book (Baker et al. 1984), for example, was not an account of all injuries in the United States, only an account of those injuries that resulted in death. Injury Severity Scales from that period categorised all injuries according to the likelihood of them leading to death. More recently, hospital morbidity was included as a recognised outcome (Better Health Commission 1986) and now, efforts are being made to quantify long term disabilities that affect injured patients long after their discharge from hospital (Maurrette et al. 1992). This new emphasis resulted in injury severity scores which categorised injury according to the likely consequent impairment (Committee for Injury Scaling 1994). Health in terms of quality-of-life after injury is now starting to be considered in the context of injury control (Rhodes et al. 1988; National Highway Safety Administration 1992; Guria 1993; Shiell and Smith 1993). 
If the public health model of injury is to remain an effective force in the control of the problem it must remain relevant to contemporary health concerns. It is imperative therefore that injury outcomes are measured in terms of a global unit of well-being suitable for use across the full range of injury types and severities. Injury scores must be developed that allow injuries to be categorised according to their potential for diminishing this quality of life.

\subsubsection{The population as the focus of public health}

Traditionally, disease prevention strategies were aimed at those people with a high risk of acquiring a given disease, on the grounds that these were the people most likely to benefit from the intervention (Rose 1985). This was the model initially accepted for use in injury control (Krauss and Robertson 1992:1021). Rose (1981) described this public health approach as the 'high risk strategy' and suggested that greater community health gains could be made if a 'mass strategy' were adopted. He demonstrated that the benefits are considerably greater if the risks of all people in a population are lowered and concluded that the mass approach is inherently the only ultimate answer to the problem of mass disease.

Injury epidemiologists were sympathetic to this view (Barry 1975) and, as exemplified by seat belt laws, have readily incorporated the mass strategy into their approach. In countries where the wearing of car seat belts is compulsory, it is compulsory for all and not just for males under the age of thirty five years. Injury workers have demonstrated their commitment to the mass strategy by introducing 'passive' interventions and 'top down' legislative sanctions.

As we have seen in the earlier sections of this chapter, injury control strategies have traditionally concentrated upon the prevention of serious and life-threatening injury. The use of the mass-strategy in injury control was introduced to reduce the risk of individuals sustaining a severe or life-threatening injury. What the injury epidemiologists have not emphasised, however, is the logical extension of the principle 
underlying the mass-strategy. If population health is the objective of injury control then injury prevention efforts should not be directed towards those injuries that carry the greatest risk of individual morbidity but towards those groups of injuries responsible for the greatest population health decrement.

The disability arising from a sprained ankle or broken finger may not be great if compared with that arising from a visceral rupture, but the ubiquity of these minor injuries may make their contribution to the total population morbidity quite substantial. If injury outcomes can be measured, not in lives lost or numbers of patients hospitalised, but in terms of a quality-of-life unit, then the outcomes of the full range of injuries could be quantified. If the morbidity in the community due to the accumulated outcomes of the minor injury is greater than the relatively small number of serious injuries, then, in terms of population health, the current emphasis on severe and life threatening injury discussed in Section 1.2 may represent a misplacement of priorities.

There is increasing evidence in the literature to suggest that non-life-threatening injuries are indeed responsible for a major part of the morbidity arising from injury. Injuries scoring AIS 1 and 2 constitute 94-97\% of all injury requiring medical attention (Schelp 1987; Sahlin et al. 1990); of patients attending hospital casualties with injury, only 3-6\% are admitted (Gallagher et al. 1984; Linqvist 1989); and of patients admitted with trauma, only $3 \%$ have an Injury Severity Score greater than 16 (Wardrope et al. 1990).

Furthermore, the health consequences of non-life-threatening injuries can be substantial. Longitudinal follow-up studies of minor cervical spine (Hildingson and Toolanen 1990) and minor head injuries (Wrightson and Gronwell 1981) have documented the long term disability that can arise from minor injury. At the population level, non-life-threatening trauma results in significant impairment and disability (Rivara et al. 1991; Maurrette et al. 1992). Further, a high percentage of non-life-threatening injuries require some time off work (Lee 1982; MacKenzie et al. 1988; Forward 1988) and the majority of time off work after injury is due to injury that 
has not resulted in hospital admission (Jansson and Jacobsson 1988; Waller et al. 1989; Waller et al. 1990). In economic terms too, it has been shown that non-severe injuries are far from inconsequential (Vipulendran et al. 1988).

The evidence described above, however, is not conclusive. Without a method of systematising minor injuries for epidemiological research the full extent of the minor injury problem cannot be quantified (Rivara 1989; Yates et al. 1991; Alexander et al. 1992). The importance of non-life-threatening injury is yet to be established.

\subsection{Conclusion}

The current epidemiological model of injury has proved to be a particularly effective framework for improving the injury-related health within communities. Further improvements in the health of populations may result from two refinements in the public health approach to injury control: injury outcomes need to be considered in terms of a global unit of well-being; and injury prevention strategies need to be specifically directed at those injuries responsible for the greatest population morbidity. To facilitate this change of emphasis, the public health impact of minor injury needs to be documented. 


\section{Chapter Two}

\section{Review of current knowledge of the public health impact of injury}

\subsection{Introduction}

It is a principle of public health that the delineation of the nature of a problem is a necessary first step in its ultimate management:

detailed knowledge of the distribution of disease and disability in the community is essential for studying the causes of disease and trends in health service utilisation, for measuring needs and outcomes, and for planning services (Coulter 1987:263).

The public health impact of injury on a society must be considered both in terms of adverse health consequences and in terms of health care resources expended. An adequate description of the injury problem documents the incidence and distribution of injury occurrence, the epidemiology of injury outcomes and the costs of treating these injuries.

Chapter One of this thesis discussed the relationship between injury and public health. This chapter presents the nature and extent of the public health impact of injury in a review of the relevant literature. It also includes reviews of the methods used to measure the dimensions of the problem and the ways in which injury is categorised.

\subsection{The injury iceberg}

The injury iceberg has been found to be a useful metaphor to describe the nature of the injury problem in the population (Wheatley and Cass 1989). The iceberg is classically divided into the categories listed in Figure 2.1. Injury fatalities represent the tip. They constitute a relatively small part of the overall problem but are clearly visible. Injuries which require hospital inpatient treatment are more numerous, less severe and less easy to quantify. Injuries which receive ambulatory care in hospital emergency departments 
and general practitioners are increasingly less severe, more numerous and less easy to quantify. Below this are those injuries which do not require medical treatment. Despite the considerable advances over recent years in our delineation of the injury problem, much of the iceberg remains unexplored. Research has concentrated upon the tip of the iceberg, leaving the vast underwater mass ill-defined and unquantified.

Figure 2.1 The injury iceberg

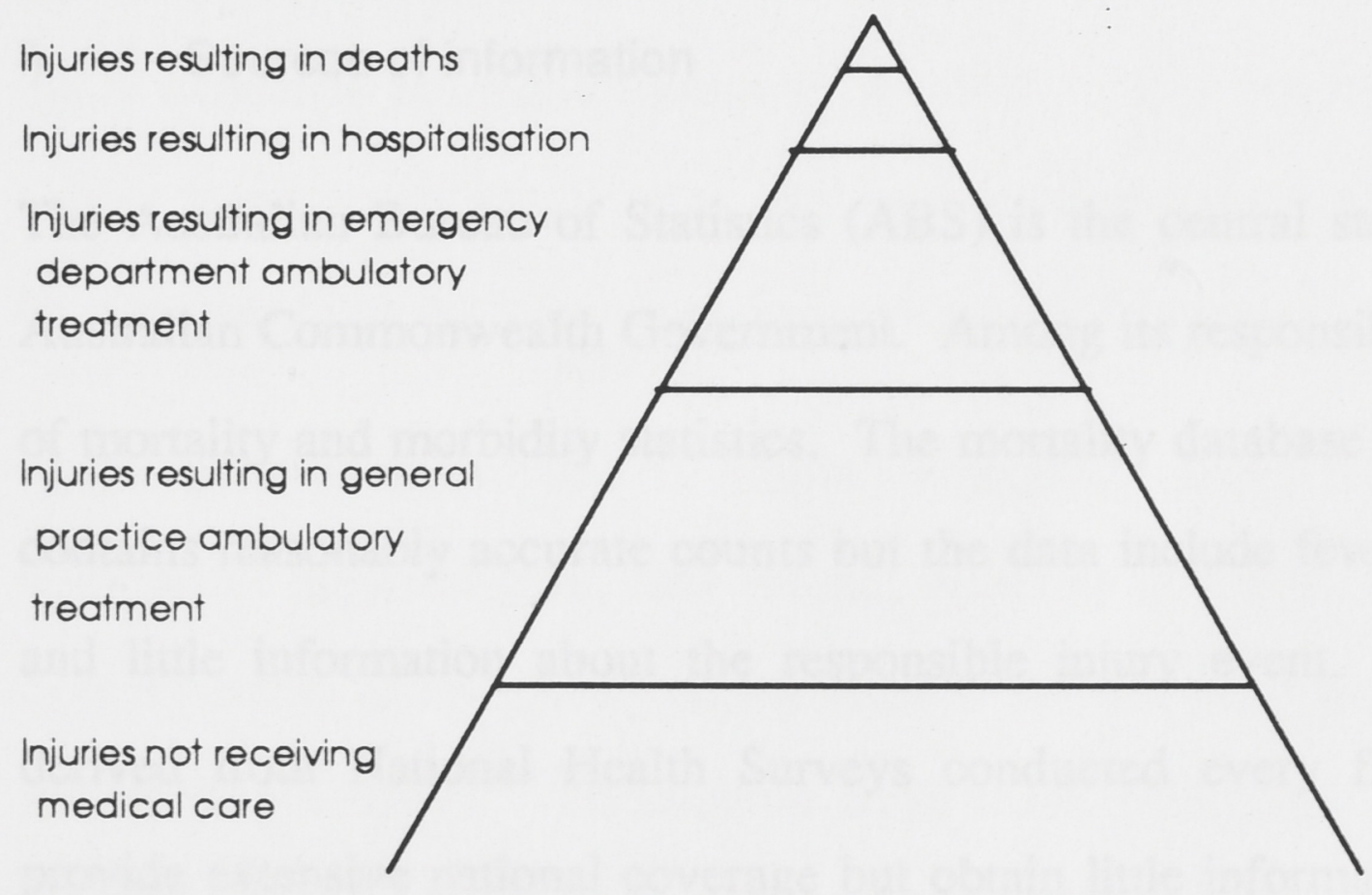

Current knowledge of the incidence and distribution of injury occurrence for each of the iceberg categories is reviewed in Section 2.3. Research into the health cost of injury is summarised in Section 2.4, and Section 2.5 describes the economic cost of injury. The scores by which injury is categorised on the basis of predicted health outcomes are presented in Section 2.6. 


\subsection{Incidence and distribution of injury}

\section{Australia}

Data concerning injury incidence and distribution come from a variety of sources. The type of information available and the quality and limitations of the data depend upon the characteristics of these sources. The review of the epidemiology of injury in Australia presented in this section begins with a consideration of the sources of information.

i) Sources of information

The Australian Bureau of Statistics (ABS) is the central statistical authority for the Australian Commonwealth Government. Among its responsibilities is the maintenance of mortality and morbidity statistics. The mortality database is national, complete and contains reasonably accurate counts but the data include few details about each death and little information about the responsible injury event. Morbidity statistics are derived from National Health Surveys conducted every five years. The surveys provide extensive national coverage but obtain little information regarding the nature of injuries sustained and injury events experienced.

Coroners' data and death certificate data are a good potential source of detailed information about injury deaths as extensive data are collected by coroners in the course of their enquires. However, in only one Australian state is the Coroner's data collected in an easily retrievable form.

The Federal Office of Road Safety conducts periodic National Road Fatality Surveys which provide comprehensive data about road crash fatalities for the years of the surveys. The Australian State Road Traffic Authorities maintain databases of information from police reports. These data include details of road crashes in which a casualty occurred, with detailed information regarding injury events. Unfortunately, the differing recording criteria between states results in national figures of uncertain 
quality. The road injury data in Australia will be considerably enhanced by a special purpose database currently being developed by the National Injury Surveillance Unit.

Each State and Territory in Australia collects information related to hospital activity. This includes data on patients' conditions (identified by ICD-9 code) and length of stay in hospital. Some attempts have been made to standardise this collection across states and most States now send data to the Australian Institute of Health and Welfare (AIHW) for aggregation into national statistics. At the present time however, the AIHW considers that only NSW and South Australia's data are complete enough to use for analysis (AIHW 1992). The usefulness of hospital morbidity data in determining injury incidence is limited (Smith et al. 1991). It is not incidence based; it reflects patient management practices as well as disease characteristics; participating hospitals change over time, and disease coding categories vary between states and over time.

Where patients are treated in hospital emergency departments but not admitted to hospital for inpatient care, data are not routinely available. Some data are available from a number of emergency departments which are participating in the national injury surveillance network. Information from these emergency departments is obtained using survey forms which are completed by injured subjects and hospital staff and which focus on the details of the injury event. Technical support for this datacollection process comes from the National Injury Surveillance Unit of the AIHW, which aggregates the data from the participating emergency departments throughout the country. The national injury surveillance network was designed to facilitate the planning of prevention strategies and it provides excellent information for this purpose. The hospitals involved in the national injury surveillance network, however, were not randomly selected. The sensitivity and representativeness of the surveillance systems operating in the participating hospitals are variable and unknown. Thus this data cannot be used for national estimates of injury incidence and distribution.

Data about occupational injuries can be derived from collections of respective State Departments of Labour. The National Data Set of Workers' Compensation-Based 
Statistics includes all people who have successfully sued for workers compensation. These statistics have been shown to be an underestimate of the total incidence of occupational injury (Harrison et al. 1989). Excluded from state databases are those employees covered under Commonwealth Workers Compensation system, those injuries which result in less than five days off work and all work injuries which occur to people not entitled to workers' compensation, ie self-employed and unofficial workers. Other problems with this database are the unknown denominators (people at risk) and the missed cases due to the filtering effect of work injury surveillance systems (Webb et al. 1989).

There are many insurance databases in Australia (eg: Transport Accident Commission database in Victoria) which are possible sources of information regarding injury occurrence and outcomes. These databases were designed for administrative purposes rather than for epidemiological research on injuries. Hence, they are limited in much the same way as the workers compensation database discussed above. Because insurance databases incompletely identify injuries in a population, they tend to reflect the experience of the included injuries rather than the population as a whole. Fire department reports and ambulance reports are also possible data sources, but these tend to focus on the function of the relevant services and, without cross-linkage to other databases, are not helpful in estimations of injury incidence.

There are no national databases which routinely collect information about minor injury. There is no routine collection of data concerning the injuries treated in general practices throughout Australia. Epidemiological studies reported in the scientific literature are, in many cases, purpose specific and rigorous examinations of particular aspects of the injury problem and as such provide invaluable information. Despite their narrow focus, they can provide insights which help to construct an overall picture. 


\section{ii) Deaths}

In Australia in 1992 there were 7,079 deaths from injury, representing a crude rate of 40 per 100,000 persons. Motor vehicle accidents accounted for 1,408 male deaths and 658 female deaths and falls resulted in 455 and 511 deaths in males and females respectively. There were 1,820 male suicides and 474 female suicides, 196 male homicides and 123 female homicides and all other external causes resulted in 1,351 and 493 deaths for males and females respectively (ABS, 1993). Injury and poisoning is the fourth highest cause of death by ICD-9 chapter heading and is the primary cause of death for people aged from 1 to 44 years (AIHW 1992).

Deaths from injury are frequently presented in terms of premature years of life lost (PYLL) (Rockett 1989). This unit is used in measuring injury consequences because it combines the total numbers of injury fatalities with the age of each person at the time of their death. In Australian males, injury results in more PYLL before the age of 65 years than any other condition, while in Australian females, injury is the second most important contributor to years of life lost (Ginpil et al. 1992).

iii) Hospital inpatient care

The national incidence of injury admissions to hospitals throughout Australia has not been identified. Hospital data from New South Wales and South Australia collected in 1988 suggest an injury and poisoning admission rate of 19.5 admissions per 1,000 persons (age-standardised to the Australian population). This constitutes the second highest admission rate for any ICD-9 category. The average length of stay in hospital was 6.1 days (AIHW 1992:40).

\section{iv) Emergency department cases.}

The incidence and distribution of injury that presents to hospital emergency departments in Australia is not available at the national level. Studies suggest, however, that the treatment of injury forms a large part of the work-load of emergency 
departments throughout the country. In 1975, a comparison of three children's hospital emergency departments showed that from $5 \%$ to $25 \%$ of all attendances were for injury (Lovell et al. 1975). A study in the Northern Metropolitan Region of Sydney in 1977 reported $50 \%$ of all casualty attendances were due to injury (Scotford and Lawson 1977).

Emergency department attendances have been described for children 14 years and younger, resident in the North-West quadrant of greater Melbourne in 1989 (Nolan and Penny, 1992). Data obtained from a regional surveillance system showed that there were 8,207 attendances recorded over the twelve month period for nonintentional injury from an estimated 150,604 children at risk. The most frequent injuries treated in the department were lacerations (23\%), fractures (21\%), bruising (16\%), sprains (11\%) and abrasions (5\%). A complete analysis of the incidence and distribution of adult injuries has not yet been published from this surveillance system though information about such injuries is regularly provided in their surveillance reports (VISS 1994).

\section{v) General practice cases}

It is generally believed that most general practitioners (GPs) treat very few injuries (Vimpani 1989; Nolan and Penny 1992) There have been two general practice morbidity studies conducted in Australia over the last thirty years which provide some information on injury. The most recent is the Australian Morbidity and Treatment Survey 1990-91 (AMTS) and only the preliminary report is currently available (Bridges-Webb et al. 1992). The earlier survey (Rowe 1976) suggested that during 1973 the average Australian GP saw 5 new injuries per week and that nationally, more than 3 million injuries received their initial management in general practice.

These studies are important contributions to knowledge of injury in general practice, however, several aspects of the methods employed limit the extent to which epidemiological generalisations can be made from the findings. First, as the 
investigators emphasise, the encounter-based emphasis of the surveys ensure that they represent "the work of GPs rather than the incidence or prevalence of disease in the community" (Bridges-Webb et al 1992:10). Second, volunteer GP samples, in the case of the early survey and random samples with response rates of less than $50 \%$ in the case of the 1990-91 study, introduce the potential for biased results. Third, as neither of the studies recorded the concurrent hospital presentations they can be used to evaluate the GP involvement in injury but cannot be used to estimate the proportion of the total injury problem treated by general practice.

There have been three subsequent pilot studies conducted in Australia that specifically address injury in general practice although results of these have not yet been published in peer-reviewed literature. (Day and Ozanne-Smith 1993; Ozanne-Smith and Brumen 1992; Taylor [personal Communication] 1994).

\section{vi) Total picture estimates}

Several overviews of the extent of injury in Australia have been published. In 1986, the Injury Task Force report to the Better Health Commission used ABS mortality statistics, South Australian hospital morbidity data and ABS National Health Survey data to estimate a national annual rate of 7,600 deaths, 300,000 admissions and 10 million medical treatments resulting from injury (Better Health Commission 1986:197). Problems with extrapolating local data and combining prevalence-based data makes this only an approximate estimate of injury occurrence. The same data sources were used in 1994 to estimate the injury burden (AHMAC/NH\&MRC working groups 1994b:9) this time calculated at 7,489 deaths, 300,000 hospital admissions, 5.7 million doctor consultations and over 10 million recent injuries, mostly of minor severity.

Two population-based estimates of injury in Australia across the full range of ages and injury types have resulted from Australia National Health Survey data. In the 1977-78 survey, $2.2 \%$ of men and $1.2 \%$ of women were reported as having sustained an injury during the two weeks prior to interview (Colditz 1983). In men, these injuries 
predominantly affected hands and fingers (22\%), legs and ankles (18\%) and backs and trunks (17\%). Injuries were similarly distributed in women (Colditz 1983). In the 1989 survey, $1.9 \%$ of the population were found to have had a new injury during the two weeks prior to interview (ABS 1992). The rigorous sampling methods and high participation rates of this sample make these studies valuable contributions to our knowledge of injury. However, inadequate case definition based on self-reported diagnoses, limits the extent to which the population estimates reflect true injury incidence.

There has been only one all-age all-injury population survey conducted which specifically addressed injury incidence in Australia (Gordon and Silverstone 1965). This was a random sample survey of 2,836 Brisbane households conducted from 1956 to 1958 . Interviewed subjects were asked to recall accidents occurring in the previous five years which resulted in injuries requiring medical attention. Detailed information was obtained regarding cause and outcomes of injury. Twenty-seven percent of males reported having sustained at least one accident over the preceding 5 years compared to $17.9 \%$ of the females. The risk of injury was greater in young males and ageing females. Only a small percentage of injury resulted in hospitalisation. Forty-seven percent of injuries occurred at home, $8 \%$ occurred at sport, $21 \%$ occurred at work, $4.5 \%$ occurred on holidays and $18 \%$ occurred on the roads. A problem with the study was that in order to obtain enough information about a relatively rare condition the study group were asked to recalled detailed events which occurred many years previously. The authors noted that the five year recall period probably led to inaccuracies in the study's results.

Problems of recall have been avoided by studies which obtain population-based data by prospectively sampling from treatment centres within defined populations. A recent study of children's injuries in the North-West Melbourne region reported injury rates of 1.4 deaths, 111 hospital admissions, and 623 emergency room treatments per 10,000 children years (Nolan and Penny 1992). This study has been one of the few which have adequately sampled from all hospitals in a circumscribed region. This study 
however was restricted to children and did not include estimates of injury presenting to general practice.

Satisfactory population estimates are obtained from hospital samples only if the sample is representative of the population and the reference population parameters are known. A series of studies conducted in a major teaching hospital in Sydney exemplify the sampling problems that arise in hospital based studies. Westmead is the principal referral centre for the Western Metropolitan Region of Sydney. In a study "designed to document the range of paediatric trauma seen in an urban environment" (Chan et al. 1989:1540), the authors note that the hospital serves a population of 1.5 million. They also note, however, that with about 8 district hospitals in the region there is "substantial referral (estimated at about 30\%) from these hospitals to Children's Hospitals out the region" and hence "this series is not representative of the range of trauma that occurs in the region" (Chan et al. 1989:1540).

There remains no adequate, population-based account of the epidemiology of injury in Australia. The incidence and distribution of minor injuries in Australia is unknown.

\section{United States of America}

As in Australia, national data sources in the USA are often an aggregation of data received from individual states. The types of databases and scope of their coverage in many ways resemble those in Australia. The National Centre for Health Statistics collects the Vital Statistics Mortality Data, the National Health Interview Survey, the Social Security Administration Survey, the National Hospital Discharge Survey and the National Ambulatory Medical Care Survey. The National Highway Traffic Safety Administration is responsible for Fatal Accident Reporting System which contains data on all police-reported fatal traffic crashes, and the National Accident Sampling System which is produced from a national sample of all police-reported motor vehicle crashes including those involving pedestrians and bicycles. The United States Consumer Product Safety Commission manages the National Electronic Injury Surveillance 
System and the Accident Investigations database. The Occupational Injury and Ilness Program administers an Annual Survey of Occupational Injuries and Illnesses, and a Supplementary data system. The National Institute for Occupational Safety and Health collects the National Traumatic Occupational Fatality database. Other databases include the Indian Health Service database, the Behavioural Risk Factor Surveillance database and special-purpose collections on fire, criminal justice, and boating injuries, and drug abuse, burns, child disabilities, child abuse, head and neck injuries, high school athletic injuries, spinal cord injuries, and poisonings. State-based data include coroners reports, Uniform Hospital Discharge Data Sets and trauma registries. There is no routine GP surveillance, and national data on injury outcome, rehabilitation, and long term disability are almost non-existent (Gable 1990; National Committee for Injury Prevention and Control 1989).

In 1989, 150,869 Americans died as a result of injuries (Department of Health and Human Services 1989). Injury is the leading cause of death among children above nine months of age in the United States (Waller et al. 1989) and injuries account for nearly one third of potential years of life lost in that country (Centres for Disease Control 1987; Rockett and Smith, 1989).

As in Australia, the incompleteness of national injury data bases in the United States makes them inappropriate for accurately identifying the extent of the injury problem in the population. However, there have been three major studies that have successfully documented the extent of the injury iceberg and these are frequently used as reference for specific work in the area.

The first of these studies involved a stratified random sample of injuries attending hospital emergency departments in North Eastern Ohio in 1977 (Barancik et al. 1983). The estimated crude trauma incidence rate reported in this study was 197 per 1000 person years. 
A study of the incidence of fatal and non-fatal injury in Massachusetts children and adolescents in 1980-81 (Gallagher et al. 1984) reported an overall incidence of 224 per 1000 person years with a ratio of emergency room visits to admissions to fatalities of $1,300: 45: 1$. The overall ratio of male to female injuries was 1.66:1.

The third major population-based study of injury in the United States (Rivara 1989) extended the identification of cases from emergency rooms to include medical centres. It was based upon a population of people aged less than 20 years who were enrolled as members of a large staff model Health Maintenance Organization. The crude rate of injuries in this population was 247 per 1000 person years, with $60 \%$ being treated in physicians clinics and the remainder in the hospital emergency room. Only $2.5 \%$ of the patients were hospitalised.

There have been very few US studies detailing the characteristics of injury in general practice. One of these (Philadelphia Injury Prevention Program 1988), estimated that only $8-16 \%$ of injuries in urban areas were treated in private physicians offices and most of these were not serious, in other areas however, the general practice component may be as high as $60 \%$ of all injuries (Consumer Product Safety Commission 1978; Rivara, 1989).

\section{Rest of the world}

In general terms, the nature and extent of the injury problem in Australia and the United States is similar to that described by authors for other parts of the world. A comparison of mortality rates in the United States France, Japan, West Germany, and the United Kingdom (Rockett and Smith 1989a and b) is presented in Table 2.1. 
Table 2.1 Mortality from injury by sex and country. Data were derived from results reported by Rockett and Smith (1989a and b) from 1980 WHO mortality tabulations.

\begin{tabular}{lccc}
\hline & \multicolumn{3}{c}{ Deaths from injury per 100,000 persons (crude rates) } \\
\cline { 2 - 4 } & Males & Combined rate & Females \\
\hline Australia & & 57 & \\
United States & 106 & & 38 \\
New Zealand & & 62 & 73 \\
France & 116 & & 28 \\
Japan & 63 & & 52 \\
West Germany & 88 & & 37 \\
United Kingdom & 52 & & \\
\hline
\end{tabular}

These results are consistent with mortality rates in other countries. For example Schelp (1987) reports 44 injury deaths occurring per 100,000 in Sweden and Sahlin et al. (1990) report a mortality rate of 46 per 100,000 in Norway. Available evidence suggests that with the decreasing problems from infectious diseases in the developing world, the importance of chronic disease and injury in these countries is increasing. Hospital morbidity throughout these regions is also extensive (Silva 1984; Elechi and Etawo 1990; Smith and Barss 1991; Mock et al. 1993).

There have been several important studies outside Australia and the United States which have documented the complete injury picture. In a Swedish study all injury visits to primary care physicians, county hospitals and regional hospitals were recorded (Schelp 1987; Schelp and Svanstrom 1987). Injury fatalities were identified from death certificates and registers. The overall injury rate before intervention strategies were commenced was 113.3 injuries per 1000 person years which is about half that described in comparable United States studies.

Subsequent Scandinavian studies using Schelp's model have produced similar results. In Norway in 1990, an injury incidence rate was 114 per 1000 people at risk with an admissions rate of 9\% (Sahlin et al. 1990). Linquist (1989) has reported an estimate of 119 accidents per 1000 person years. 
In Jerusalem, results obtained from a population-based study of children aged 0-17 (Gofin et al. 1991) were similar to those in Scandinavia. All hospitalisations and deaths were recorded and a $25 \%$ sample of attendances to emergency departments was obtained over a twelve month period. The incidence of injury presentations to emergency departments was 98 per 1000 person years and hospitalisations were 2.5 per 1000 person years. Thus there was a ratio of one death to 43 hospitalisations to 1,732 visits to hospital. The male to female rate was $2: 1$ for emergency room presentations and 1.8:1 for admissions.

The incidence and distribution of serious and life-threatening injury is well documented in the literature. The described research demonstrates the magnitude of this international problem. Little, however, is known about the epidemiology of minor injury in Australia or overseas.

\subsection{Health costs of injury}

As noted in the introduction, the public health impact of injury on a society involves both the incidence and distribution of injury and the ongoing adverse health consequences of these injuries. Adequate description of the injury problem must, therefore, include an account of the epidemiology of injury outcomes. This section discusses the literature that provides information relating to the outcomes of injury.

\subsubsection{Outcome methodology}

Traditionally the outcomes used in injury research include deaths, potential years of life lost (PYLL), whether hospitalised, number of days in hospital and time off work after injury. These are objective measures and are easily obtainable. With the more recent emphasis on including assessment of disability in evaluations of health conditions (Anonymous 1986), studies have begun to consider the more sophisticated health outcomes of permanent impairment and consequent disability. 
In 1980, the WHO published the International Classification of Impairments, Disabilities and Handicaps (ICIDH) for categorising disability (WHO 1980). In this system, impairment was defined as a disturbance (either temporary or permanent) to the body's mental or physical structure or functioning and disability was defined as a reduction or loss in a person's functional capacity resulting from an impairment. The ICIDH has been used by several injury researchers (Chalmers et al. 1989; Lodge et al. 1990; Maurrette et al. 1992). However, the lack of reliability and validity of this system have prevented its universal acceptance (Anonymous 1986).

Disability measurement based on activities of daily living and general functional status have been used for many years by researchers of the aged and chronically diseased (Katz et al, 1983; Lawton et al. 1982; Eisen et al. 1980). These measures have been logical choices for injury researchers in the area of injury outcome assessment and have been used with variable success (Wesson et al. 1989; Rivara et al. 1991; Morris et al. 1991). Also, injury researchers have adapted existing functional capacity instruments or constructed new schedules to suit the specific needs of individual projects (MacKenzie et al. 1988; Glancy et al. 1992; Waller et al. 1990; MacKenzie et al. 1986).

Functional limitations and disability, however, do not encompass the full range of consequences of injury and measures of disability therefore do not fully assess injuryrelated morbidity. Health is defined by the World Health Organization in its constitution as "A state of complete physical, mental, and social well-being" (WHO 1984). Morbidity, then, can be taken as any compromise to this state. Last's definition of morbidity as "Any departure, subjective or objective from a state of physiological or psychological well-being" is essentially the same (Last 1988:125). The public health measurement of morbidity should capture three dimensions: number of persons involved; the illness; and the duration of the illness. Thus, to quantify the morbidity in a population arising from injury, one must use an index that combines the dimensions of persons, well-being and time. The desirable outcome measure for today's definition 
of health is a health status index based on quality adjusted life year units (QALY) (Schwartz et al. 1993).

Health status indexes are constructed, first by ascertaining the health profile of an individual, then by scaling the health profile to reduce it to a single number which represents the individual's global health-related quality of life. This process, in effect, quantifies and weights the value of different health states in order to reduce the complex health information to a simple summary score. There are several means by which the value weights may be devised. The simplest of these involves 'category scaling'. Chosen individuals are given descriptions of different states of health and asked to locate each one on a scale of numbers within a stated range, where equal differences between the numbers correspond to equal gradations in the overall health status. Magnitude estimation and preference ranking are similar scaling processes.

A second technique is 'equivalence' estimating. This aims to identify a judge's point of indifference between the health value of a hypothetical standard group of unwell people and another group of less well people. The judge is asked to identify the number of less well people who should be cured of any given condition for this to be the equivalent of curing the standard group. From this equivalence, numerical values of the different health states are inferred.

The 'standard gamble' is a third method of deriving weights. In this process, an individual is presented with a choice between a given condition and a gamble between perfect health and death. The subject is asked to identify the odds that would make the gamble equivalent to the stated medical condition. A variation of this concept is the 'time-trade-off' method which confronts a judge with a choice between differing numbers of years in differing states of health.

None of these systems has achieved universal acceptance and conflicting results are obtained from these several methods (Brooks 1986:44). The advantages of the category scale are that it is easy to administer and intuitively easy for the subjects to understand. The validity of the results, however, has not been demonstrated. The 
lottery based techniques, ie the standard gamble and the time-trade-off questions, are favoured by decision theorists and economists because these methods have roots in fundamental axioms that underlie the utility model of economic choice. In practice, however, these utility weights are not easily obtained.

The second unresolved issue with these scaling methods is who is to set the values for the various health states. The obvious choices are between experts, population samples or the unwell individuals. Although all of these have been used, there is still debate about the most appropriate method. It has been suggested, for example, that self-rating is limited by subjectivity (Liang and Jette 1981:85) and assignments of value by panels of experts are inconsistent and possibly biased (Kirwin et al. 1983). Having a population sample of subjects attach values to a range of hypothetical conditions can also be criticised on the grounds that people who have not experienced the conditions could not know the true worth of these health states.

There are several general health status indexes which can be used for measuring the outcome of injury. The best known of the QALY indexes are the Rosser-Kind index (Rosser and Watts 1972; Gudex and Kind 1987) the Euroqol (The Euroqol Group 1990), the index developed by researchers at McMaster University (Torrance et al. 1982; Torrance 1987) and the Quality of Well-Being Index (Kaplan 1989).

The Rosser-Kind index uses a visual analogue scale to derive weights. The validity and reliability of this scale has not been demonstrated (Carr-Hill 1991). The Euroqol also uses a visual analogue scale to obtain value weights for the various health states experienced by the subject. Pilot work on the Euroqol in Australia found this measure to be imprecise and ambiguous and substantial revision of the questionnaire was advocated (Snow 1991).

Utility weights for the McMaster index are derived from questions based upon principles of the standard gamble. The authors of this index favour the standard gamble and time-trade-off approaches because they are "based upon a solid body of theory and a set of compelling axioms" (Torrance 1987:599). They nevertheless 
acknowledge that the instrument used to obtain these weights is time consuming and furthermore has to be adapted to each particular study. These weights cannot be obtained from mail, self administered questionnaires.

The Quality of Well-Being (QWB) scale is a more developed instrument and has withstood repeated psychometric evaluation over the many years since it was introduced. The QWB uses a structured interview to classify the respondent's function over the preceding six days. Any symptoms or medical conditions experienced over this time are also recorded. The questions are recorded in terms of what the subject did rather than what they thought they were capable of doing. Function is divided into mobility, physical activity, social activity and self care and the functional status categories range from full independent function to death. The questions were originally developed from the US National Health Interview Survey and the Social Security Administration Survey, but have since undergone extensive revision.

For each of the functional levels a value weight is attached, reflecting social preferences for each of the health components. The weights were derived from a population sample of 867 subjects who rated 500 items on an interval scale. They are scaled such that 'one' represents full health, 'zero' represents death and all intermediate health states fall somewhere in between. The QWB is scored by subtracting the value of each health compromise from one and total amount of health loss is calculated by multiplying the resulting score by the number of years spent in this state. The reliability of the estimated preference weights has been well documented and has been reported as high as 0.90 (Kaplan and Bush 1982; Anderson et al. 1988; Anderson et al. 1989). The instrument has high content validity and displays satisfactory construct validity (Kaplan et al. 1976). It is stable over time and generalisable (Kaplan et al. 1978).

Although these QALY-based general health indexes such as the QWB scale can be used to measure the outcome of injuries, they have rarely been used for this purpose (Holbrook et al. 1994). On the other hand, the principles underlying the units currently used for the measurement of injury outcome do not differ greatly from those of the 
QALY measures described above. For example, the QALY technique of combining the dimensions of 'numbers of people', 'health states' and 'time in a given health state' is already incorporated in the PYLL unit where 'numbers of people', 'death' and 'time' are combined. The difference between the two lies in the use of a dichotomised health state in PYLLs (ie life-death) compared with the use of a scale of health states in the QALY measures.

Similarly, the scaling of health states is already a standard tool in injury epidemiology. Most existing Injury Scales and Severity Scores (eg AIS) have been constructed by panels of experts assigning value weights from a category scale to injuries on the basis of the predicted consequent health state.

The World Bank and World Health Organization have combined mortality and morbidity data in a unit called 'Disability Adjusted Life Years' (DALYs) to facilitate international health comparisons (World Bank 1993). This process involved the assigning of disability severity weights to the various non-fatal conditions by a panel of experts. DALYs lost due to injury were estimated by adding discounted life years lost from injury fatalities to the figure obtained from combining the incidence of non-fatal injuries with age at onset and duration of disability.

In 1992 the National Highway Traffic Safety Administration (NHTSA) presented a preliminary report of a similar scale for measuring the outcome of injuries in functional capacity-adjusted life years. The proposed Functional Capacity Index will be a measure of the relative degree to which an injured person is unable to function at their pre-injury level. Ten functional dimensions have been identified. The researchers plan to use the simple multi-attribute utility rating technique (Torrance et al. 1982) to scale the different levels of each dimension and the different dimensions to result in a single index that represents the whole body functional capacity of the injured person. Final reports of this process have not yet been published.

It can be seen then, that considerable advances have been made towards the use of QALYs for the measurement of injury outcomes. Currently, however, there is no 
simple, valid, QALY instrument suitable for use in large-scale surveys of injury outcome and there have been no population-based studies which have empirically measured the outcome of injury in QALY units.

\subsubsection{Outcomes of injury}

An account of the natural history of a health condition is an integral part of its epidemiological description. Within the limits of the methodologies discussed in the previous section, a summary of the current knowledge of injury outcomes is presented below.

\section{Australia}

There are no Australian population-based studies of the outcome of injury across the range of injury types and severities. Few descriptive epidemiological studies of injury include a consideration of the natural history of injury recovery, nor have there been any specific attempts to quantify the epidemiology of injury-related morbidity and disability in the community. The need, however, for further research into the long-term outcomes of trauma in the community has been noted (Lyle et al. 1990). The followup studies of injury in Australia are essentially confined to hospital case series for the purposes of evaluating and aiding clinical management, (eg Day et al. 1994) though rare exceptions to this rule can be found (eg Balla et al. 1970). Neurotrauma and its outcomes, however, are well researched (Ford 1976; Simpson et al. 1984; Burke et al. 1985; Jones et al. 1986, Cain et al. 1989; Ryan 1992) and it has been shown that neurotrauma was responsible for $70 \%$ of all road accident deaths and $50 \%$ of all deaths from external causes. About $51 \%$ of neurotrauma patients died, $1 \%$ survived in vegetative states, $14 \%$ had severe disability $13 \%$ had moderate disability and $22 \%$ made a good recovery (Ring et al. 1986). 


\section{United States of America}

In the United States, as in Australia, the paucity of information about injury outcomes is well recognised:

Little is known about the less severe injuries that cause most of the visits to physicians and restriction of normal activities. The long term consequences of what may appear to be minor injury are essentially unknown (Committee on Trauma Research 1985:34)

National data on injury outcome, rehabilitation, and long term disability are almost non-existent (National Committee for Injury Prevention and Control, 1989:38).

As a consequence there have been a growing number of studies in recent years which address this problem in the United States. Generally, the studies concentrate on outcomes of those patients who sustained severe trauma (Frutiger et al. 1991; van Aalst et al. 1991; Morris et al. 1991; Glancy et al. 1992; Ward et al. 1992).

However, several authors in the last decade have drawn attention to the surprising amount of disability that arises from minor injuries (Yates et al. 1991:326). Early studies noted that non-life-threatening injuries to the extremities could lead to long term disabilities (Nagel and States 1977; Levine 1987). Long term sequelae in patients with apparently mild head injury had also been noted (Wrightson and Gronwell 1981; Rimel et al. 1981; Leininger et al. 1989). The extent of the problem was highlighted in an incidence-based disability study in collegiate sport players (Lanese et al. 1980) which found that $42 \%$ of males and $39 \%$ of females in the sample had sustained an injury during a sporting event in the previous year which resulted in missing a match or practice. In a study of functional recovery after trauma (MacKenzie et al. 1988), only $63 \%$ of those with minor injuries of the extremities had returned to work within the year. In a consecutive sample of 495 patients who received primary care in Northern Vermont for injuries sustained during wood-related construction, Waller found that six months post-injury, patients averaged 11.6 days of disability for work, 10.3 for home activities and 13.1 days for recreation (Waller et al. 1990). In a 1991 population-based incidence study of children, $55 \%$ were found to have some disability at one week after 
their injury occurrence, and 3.8\% still had some disability after one month (Rivara et al. 1991).

\section{Rest of the world}

The paucity of information available on the characteristics of the problem of disability and handicap resulting from accidents was noted by the symposium on the Epidemiology of Accident Traumas and Resulting Disabilities 1981 (WHO 1981). Studies of injury outcome in England, however, have a strong tradition. In particular, a paper by Bull (1985) on disability from road accidents is the essential reference in much of the injury-related disability work throughout the world. In this paper he analysed the disabilities suffered by survivors of traffic crashes who presented to hospital for treatment of their injury. He found $28 \%$ of the inpatient casualties and about $3 \%$ of the ambulatory patients had some level of permanent disability.

As in Australia and the USA, outcomes after severe head injuries in other countries have been well examined. As early as 1967 it was estimated in England and Wales that about 1,000 patients were leaving hospital each year with permanent brain damage (London 1967). In 1976, Jennett presented two cases series from Glasgow and the Netherlands of outcomes of patients hospitalised with severe head injury and evidence of at least six hours of coma. The results of these studies are similar to those described by Ring et al (1986) in Australia (Jennett 1976).

There have also been several studies looking at outcomes after a range of less severe injuries. In 1982, Lee demonstrated that patients with relatively minor fractures can suffer functional limitations after the bone has healed. He followed a consecutive series of 171 patients with a diagnosed fracture seen at a trauma clinic at a general hospital in South-East England. Forty-seven percent had not returned to work four weeks after the injury and $5 \%$ had not done so by 12 weeks. In studies of people involved in personal injury claims litigation, Cornes et al $(1985 ; 1986)$ noted that the medical reports anticipated at least some residual disability in approximately $95 \%$ of 
the claims. In 1981, a study of 182 hand injuries drawn from the general population illustrates the work related consequences of these injuries (Johns 1981). Median time off work was three weeks, with one third of cases requiring more than six weeks off work. In a recent study (Pennie and Agambar 1991) of a consecutive sample of patients presenting to emergency departments of the Wirral health authority with 'whiplash', $14 \%$ were found to have had continuing problems 5 months after injury.

Scandinavian studies have provided important information on community-based disability arising from injury. Janson et al (1988) conducted a study of medical consequences of work-related accidents occurring in a 12 month period on 2454 Swedish farms. There were 163 injured patients observed and six percent were admitted to hospital. Seventy-five patients had temporary disability for a combined total of 2,431 days and disability was permanent for $4 \%$ of these patients (Jansson and Jacobsson 1988). In a study in Sweden 93 cases of traffic crash soft tissue injury to the spine were followed prospectively for a period of two years, at which point $43 \%$ had residual disability (Hildingson and Toolanen 1990).

In 1992, a French group reported an attempt to evaluate both the frequency and course of impairments, disabilities and handicaps resulting from trauma (Maurrette et al. 1992). The sample was 1005 trauma patients admitted to three hospitals in a defined region. Patients who remained in hospital greater than two days were over-sampled. Patients were followed at six months and, if not recovered, again at 12 months. Of the follow-up sample, those with less severe injuries were differentially lost to follow up. The study does not therefore provide a population-based estimate of outcome from injury but provides both a useful methodology and an indication of the degrees of impairment, disability and handicap that can result long after injury event. The authors note that a universally applicable means of assessment and classification of disability is urgently needed. Minor trauma was not the specific subject of this study, but it was found that even minor trauma resulted in marked impairment and disability. 
There have been two theoretical studies conducted in New Zealand to evaluate the outcome of injuries at the population level. In 1990, Guria used New Zealand hospital data and findings from a British study linking percentage disability to length of hospital stay to calculate total social costs of disabilities from traffic injuries. In 1993, the same author looked at expected loss of life quality from traffic injuries requiring hospitalisation using a theoretical model. Neither project involved the prospective study of individuals to identify injury outcome.

It is clear from the literature that considerable efforts are now being made in the area of injury outcome assessment. There remains, however, little information regarding the nature and extent of morbidity arising from minor injury.

\subsubsection{Predicting outcomes}

In the last ten years, there has been a persistent search for attributes of the injury or injured persons which might be used to predict outcomes. The ability to identify those injuries which are likely to lead to long term health deficits is an important aid to controlling the community burden of injury. These injuries which result in major morbidity can be specifically targeted by injury prevention programmes. Acute medical management of these injuries may be improved to reduce the long term complications, and extra rehabilitation can be mobilised on the patients' discharge from hospital to reduce the amount of consequent disability.

In 1985, a study of hospitalised traffic injury patients demonstrated that, for similar kinds of injuries, disabilities were more severe in older patients and in those patients with head or lower limb injuries (Bull 1985). In 1987, Mackenzie followed a sample of trauma patients for one year after their injury. Head and spinal cord injury was a strong predictor of failure to return to work. Low return rates were also noted in patients whose most severe injury was to a lower extremity.

In 1992, a population-based sample of outpatient injured children less than 20 years of age was used to develop empirically a set of predictors of functional disability arising 
from injury (Alexander et al. 1992). At one week and one month after injury the children completed functional status assessment measure developed by the Rand Insurance study. Subsequent mathematical models with age, location of injury and type of injury showed only the latter to be a consistently significant predictor of impairment.

In 1993, Mock reported a study of disability in patients admitted to a rural hospital in Ghana (Mock et al. 1993). He used multiple logistic regression to model the development of a major disability after injury. The independent variables were injury type, mechanism of injury region of principal injury, age, sex, referral from an outlying clinic and development of inpatient complications. The strongest predictors were body part injured and type of injury.

Personal income, education level of injured person and social support have been shown to correlate with post-injury employment status (MacKenzie et al. 1987). These findings were supported by another study (Brewin et al. 1983) which found that patients who showed rapid recovery relative to the severity of their injuries were more often married, had greater job satisfaction and were less likely to be receiving income support from their employers. Mackenzie et al. (1988) studied 597 surviving trauma patients for a year after their injury event. Fifty percent of these had no activity restriction at the end of this period but only $57 \%$ of those patients who were working full time before the injury had returned to work by one year afterwards. Predictors of return to work included higher eduction level, white collar employment, higher preinjury income, and good social support.

MacKenzie et al. (1989) noted that mean length of stay in hospital following injury was $69 \%$ higher for those with pre-existing disease compared with those without. The size of the effect varied with patient age and injury severity. Pre-existing disease has also been found to be a predictor of mortality, independent of age and injury type (Milzman et al. 1992). The effect of pre-existing disease on long term effects of non-fatal injury are inadequately characterised. 
In summary it seems the predictors of disability are not well understood. Body-part injured and type of injury appear to be the most consistent predictors of long-term disability arising from injury. Age and pre-existing disease also needs to be considered.

\subsection{Economic costs of injury}

"One way to assess the importance of a health problem to a society is to calculate its (economic) costs" (Langley and McLoughlin 1989:250). As with assessments of morbidity arising from a given disease, identifying the total cost of an illness is an important step towards understanding the extent of the problem. In the classic 'costof-illness studies' (Shiell et al. 1987) the economic burden is assessed in terms of all costs that occur both within the health system and outside it.

The costs involved in organising and operating the health care of injury together with the patient's out-of-pocket expenses relating to the treatments are referred to as direct costs. Direct costs account for about $30 \%$ of the total lifetime cost of injury (Rice et al. 1989), though for certain groups this percentage is considerably lower. A recent Australian study (Bureau of Transport and Communications 1992) suggests that for transport injury, direct medical costs constitute only $1.8 \%$ of total injury-related costs.

Indirect costs are those that relate to time lost from work, pain and suffering. The third group of costs involves those expenses borne externally to the health sector, the patients, and their families.

Because of the nature of health care financing it is often difficult to identify specific costs and to partition overheads between disease groups. Charges for treatment of injury are often used as a proxy for costs in economic evaluations, but because of cross-subsidising, these cannot be seen as equivalent (Finkler 1982).

A further cost which is sometimes calculated, is based on an attempt to value the quality of life lost from injury Thompson (1986). As with assessments of morbidity arising from injury, cost estimations can be based on either prevalence or incidence 
data. Incidence data is the appropriate choice when interest lies in the anticipated future consequences of current developments. These types of data are suited to the policy maker who has the task of designing strategies for injury prevention and control. It has been argued that calculating a total bottom-line cost of illness does not provide enough useful information to enable appropriate comparisons and predictions to be made, and that, as economic evaluation in an area becomes more sophisticated, emphasis should be on cost-effectiveness, cost-benefit and cost-utility studies (Shiell et al. 1987). In the injury field the emphasis still remains largely on total cost estimations (Van Doorslaer and Bouterr 1990), however "A knowledge of the costs of accidents can enable a judgement to be made of the cost-effectiveness of a proposed countermeasure" (Andreassand 1985).

\section{Australia}

To date, there are no published Australian incidence-based, population estimates of the economic cost of injury across the range of injury types and severities. A figure of A $\$ 1,320$ million currently quoted in the National Goals and Targets report (AHMAC/NH\&MRC working groups 1994) has been derived from unpublished data from the Australian Institute of Health and Welfare.

In the field of road traffic research, economic outcomes of injury have been better explored. In 1971, in a comprehensive study of the cost of motor vehicle collisions in the Australian Capital Territory was undertaken in which it was estimated that the total costs amounted to $2.3 \%$ of gross product of the region (Butlin 1971). Further studies of the cost of motor vehicle collisions followed (Atkins 1981; Somerville and McLean 1981; Andreassand 1985; Steadman and Bryan 1988; Giles, 1988). Recent estimates of the total direct costs of road accidents in Australia put the figure at \$239 million (Bureau of Transport and Communications 1992), and work has now started on the relatively new area of measuring the cost-utility of motor vehicle accident prevention (Shiell and Smith 1993). 


\section{United States of America}

The development of cost-of-injury analysis in the United States is considerably more advanced. In 1985, it was reported that injuries result in approximately 3.6 million hospital admissions and attention was drawn to the unknown long-term costs of these conditions (Committee on Trauma research 1985). This issue was addressed in a series of injury outcome cost studies in samples of hospitalised patients (MacKenzie et al. 1988; MacKenzie et al. 1988; MacKenzie et al. 1990). More recently, the cost of nonhospitalised injuries has also been examined (Waller et al. 1990). Further developments included an incidence-based study of cost of injury by cause calculated in an industrial population in the US using insurance data (Tsai et al. 1989) and the use of the 1980 National Medical Care Utilisation and Expenditure survey to place the costs of injury in the context of all medical costs (Harlan et al. 1990). Perhaps the best overview of injury costs in the United States is to be found in the book Cost of Injury (Rice et al. 1989). For the 57 million people injured in the USA in 1985 estimated direct costs were US\$790 per injured person.

As in Australia, there has been much work in the United States that concentrates on measuring the economic consequences of road traffic crashes. The comprehensive cost of motor vehicle collisions in 1988 was estimated at $\$ 333$ billion (Miller et al. 1991). The direct costs of motor vehicle crash injuries have also been estimated by body region and severity (Miller et al. 1990; Miller et al. 1993; Miller, 1993). The savings of motor vehicle safety investment have also been demonstrated (Miller et al. 1989).

\section{Rest of the world}

Published details of the cost of injury in other parts of the world are limited but the available studies provide important information. In Germany, traffic accident data within the workers' compensation database has been used to develop tools for costbenefit studies (Zeidler et al. 1993). In Britain it has been shown that lower limb injuries play a major role in the total cost of vehicle collision injuries (Ward et al. 
1992). A study of the cost to the National Health System of accidents to children in a region of England demonstrated the significant resources spent on apparently minor injury (Vipulendran et al. 1988).

Several New Zealand studies have included estimates of the national cost of injury. Langley estimated a total direct economic cost of NZ\$425 million in 1983, representing NZ\$133 per head of population (Langley and McLoughlin 1989). The estimate was based on data from a prevalence study that combined the average cost per hospital inpatient bed day and emergency department visit, together with costs incurred by the New Zealand Accident Compensation Corporation.

Using a national data set which included all primary injuries from traffic accidents requiring hospitalisation, Guria (1990) used length of stay in hospital to predict long term disability and hence costs of traffic injuries throughout the country. He considered total costs to include inpatient treatment costs, costs of temporary incapacitation and cost of permanent disability. The standard New Zealand estimates of average hospital costs and productivity and life-worth were combined with estimates of disability from inpatient days and utility estimates of disability to construct a national figure of NZ\$516 million or NZ\$154 per person when life was valued at $\$ 500,000$ (Guria 1990).

In terms of economic cost, the public health importance of injury has been extensively described. It is clear that an economic evaluation is an essential component of any assessment of the public health impact of minor injury. This evaluation has not yet been performed for an Australian population.

\subsection{Conclusion}

This review of the literature leaves little doubt that injury is a public health problem of major importance. The available information, however, is largely restricted to detail about serious and life-threatening injuries. In particular: 
- The general practice component of the injury problem has not been adequately described. The common belief that general practitioners see few injuries is not based upon results of thorough research.

- There have been no population-based studies of the incidence of injury in Australia which have included all medically treated injuries. Estimates of injury in Australia may understate the frequency of non-life-threatening injury.

- there is no measure currently available for quantifying outcomes of injury over time in quality-of-life units that is suitable for use in large-scale surveys.

- few studies have attempted to quantify, at a population level, the morbidity arising from injury across the full range of injury types and severities.

- there are no studies in Australia which describe the economic costs of minor injury.

The public health impact of minor injury has not been adequately described. 


\section{Chapter Three}

\section{Outline of research project}

\subsection{Introduction}

Chapter Two has shown that injury is a public health problem of major importance. The nature and extent of this problem is well understood with respect to those lifethreatening injuries at the tip of the injury iceberg. However, little is known about the public health impact of minor injury. The extended public health model of injury discussed in Chapter One suggests that further research on minor injury should be conducted. This chapter outlines a research project undertaken to establish the importance of minor injury as a cause of health loss in a community.

\subsection{Aims of the thesis}

The aims of this thesis are to:-

1. Explore the role played by general practitioners in managing injury in Australia.

2. Estimate the incidence of injury in a population across the full range of injury types and severities.

3. Evaluate the ACT injury surveillance system as method of identifying a representative sample of injured people.

4. Devise a valid and reliable measure of injury outcomes consistent with the WHO definition of health and which is able to be used in large scale epidemiological surveys.

5. Estimate the incidence-based injury morbidity in the Australian Capital Territory (ACT). 
6. Estimate the incidence-based economic cost of acute treatment of minor injury in the ACT.

7. Construct an injury scoring system that enables minor injury to be categorised and ranked on the basis of its potential for resulting in health loss.

- and thereby to test the hypothesis that minor injury results in the major part of public health burden associated with injury.

\subsection{Overview}

The project undertaken to address the aims of this thesis involved seven interdependent studies. Each of the seven studies addresses one of the aims and each is described in its own chapter (Chapters Four to Ten). In Chapter Eleven, the information obtained from these studies is used to test the major hypothesis of the project. The final chapter of the thesis also includes a discussion of the limitations and implications of the findings.

All studies were conducted with the approval of the Australian National University Ethics Committee and the ACT Health Institutional Ethics Committee.

\subsubsection{Setting}

The project was conducted in the Australian Capital Territory (ACT) between 20th January 1992 and 20th January 1993. The ACT is a geographically circumscribed area of 250 square kilometres in south eastern Australia that includes the nation's capital city, Canberra. The population consisted of 296,000 people residing within the borders of the Australian Capital Territory at any time during 1992 (ABS 1993). This population is a geographically circumscribed community with high literacy levels, high socio-economic status and minimal heavy industry. 
It is served by three hospitals (only two of which have emergency departments) and 273 general practitioners. Woden Valley Hospital is a teaching hospital located in the south of Canberra and is the trauma centre for the ACT and surrounding region. Calvary Hospital, which is located in Canberra's northern suburbs, would typically see as many injuries as Woden Valley but of less severe categories. All medically treated injuries occurring within the ACT border, to ACT residents, present at one or other of these medical service providers.

The possibility of conducting a population based study of injury in the ACT has been recognised previously (Pearn and Nixon 1981). The community is geographically circumscribed, with well-documented population characteristics and case finding from the two casualty portals gives a complete population overview for a large urban population.

\subsubsection{Studies undertaken}

As discussed in Section 2.3, little is known about the numbers of injuries attending general practitioners (GPs) for initial treatment. The study described in Chapter Four was conducted to provide background information to assist the planning of a population-based study of the incidence of injury. This study explores the role played by GPs in the management of injury in Australia. It identifies the proportion of injured patients who attend GPs for initial management of their injury and the characteristics of those patients who choose this place of treatment. The study involves secondary analysis of data from the 1989-1990 National Health Survey (ABS 1992).

Chapter Five describes the incidence and distribution of injury in the ACT. A population-based study was conducted using random systematic samples of medical service providers within a defined population. A sample of patients presenting for first treatment of a new injury was taken from each of the emergency departments and from 24 of the general practitioners within the ACT. For each patient, age and sex were recorded, as were nature of injury, body-part injured and whether the patient was 
admitted to hospital for treatment of the injury. This study provided a detailed picture of the epidemiology of injury in the study population throughout the study period and thereby provided reference data for the other studies conducted within this population during the year.

Chapter Six describes the Australian Capital Territory Injury Surveillance and Prevention Project (ACTISPP) and evaluates the extent to which the ACTISPP database is representative of all injuries treated in emergency departments of the ACT. This is an important background study to the injury outcome studies described in the subsequent chapters, as all the follow-up samples were subsets of the ACTISPP sample. The properties of the ACTISPP database were assessed by comparing the characteristics of those injured subjects registered by ACTISPP with those registered in the incidence and distribution sample described in Chapter Four.

Chapter Seven describes the development of The Health Consequences of Injury Questionnaire (HCIQ). This is an instrument for measuring the outcomes of injury in quality-of-life units for use in large scale epidemiological surveys. The development of the HCIQ involved a pilot study, a validity study using the Quality of Well-Being Scale as the criterion standard, and a test-retest reliability study. The samples for these studies were sub-samples of patients registered in the ACTISPP database. The HCIQ was used in the studies described in Chapters Eight and Ten.

Chapter Eight describes a prospective follow-up study. The objectives of this study were to quantify the outcome of injuries in the Australian Capital Territory during 1992 and to calculate the contribution made by minor injury to the total consequent morbidity. The main outcome measure used was "health loss due to injury" as measured by the HCIQ. Total health loss over the period of recovery was obtained by means of repeated mailings of the questionnaire at intervals of one week, six weeks, three months and six months after the injury occurred. Those not recovered by six months were censored. The total health loss over the recovery period was obtained for each subject and, using weights derived from the incidence study, estimates were made 
of the total morbidity in the study population arising from injury which occurred during 1992. In order to identify those factors responsible for the greatest share of this morbidity, the health loss was stratified by age, sex, nature of injury, body-part injured, severity score and whether admitted to hospital.

Chapter Nine deals with the estimation of an incidence-based estimate of the economic cost of medical management of acute minor injury in the study population. This includes establishing the cost of treating minor injury in hospital emergency departments as well as the cost of treating acute injury in general practice. Details of treatments for injuries presenting to hospital emergency departments were obtained from the incidence study described in Chapter Five and the follow-up study described in Chapter Eight. Cost of these treatments was obtained from the 1992 Medicare Schedule (Commonwealth Department of Health 1992). The management of acute injury in general practice was identified by means of secondary analysis of a preexisting 1990-91 general practice database (Bridges-Webb et al. 1992). The details of this secondary analysis are described. The cost of these treatments was obtained from the 1991 Medicare Schedule (Commonwealth Department of Health 1990). The estimated average cost per case was inflated by $2.4 \%$ to account for inflation between 1990 and 1992 and then multiplied by the number of ACT general practice cases identified in the incidence study to estimate the total injury cost in the ACT during 1992.

Chapter Ten addresses the final aim of the project, that is to construct an injury scoring system that enables injuries to be categorised and ranked on the basis of their potential for loss of well-being. The chapter describes the development and characteristics of an injury scoring system from data obtained in the prospective study of injury outcomes (Chapter Eight). The health loss at the time of accident was scored for each injured person. The median score for each injury type was designated the injury severity score for that injury type. The accumulated health loss over time until recovery for each injury was calculated and the median outcome score for each injury type was 
designated the injury morbidity score for that injury. The injury severity scores and injury morbidity scores were tabled by injury type to produce a Minor Injury Scale.

Table 3.1 summarises the research project and illustrates the relationships between the aims of the thesis and the individual studies undertaken. 
Table 3.1 Summary of the research reported in the thesis.

\begin{tabular}{|c|c|c|c|c|}
\hline Chapter: Thtle & Aim & Study design & Samples & Analysis \\
\hline $\begin{array}{l}\text { 4: Injury and general } \\
\text { practice in Australia }\end{array}$ & $\begin{array}{l}\text { To identify the importance of the } \\
\text { role played by general practitioners } \\
\text { in managing injury in Australia }\end{array}$ & $\begin{array}{l}\text { Secondary analysis of the } \\
1989-90 \text { National Health Survey }\end{array}$ & $\begin{array}{l}\text { Multi-stage sample survey } \\
\text { of the Australian population }\end{array}$ & $\begin{array}{l}\text { Frequencies, cross- } \\
\text { tabulations and logistic } \\
\text { regression }\end{array}$ \\
\hline $\begin{array}{l}\text { 5: Incidence and } \\
\text { distribution of injury in } \\
\text { the Australian Capital } \\
\text { Territory }\end{array}$ & $\begin{array}{l}\text { To estimate the incidence } \\
\text { of injury in the Australian } \\
\text { Capital Territory }\end{array}$ & Descriptive incidence survey & $\begin{array}{l}\text { Random and systematic samples from } \\
\text { service providers in the Australian Capital } \\
\text { Territory, ie: general practice sample (i) } \\
\text { and emergency department sample (ii) }\end{array}$ & $\begin{array}{l}\text { Frequencies and } \\
\text { cross-tabulations }\end{array}$ \\
\hline $\begin{array}{l}\text { 6: Australian Capital Territory } \\
\text { Injury Surveillance and } \\
\text { Prevention Project (ACTISPP) }\end{array}$ & $\begin{array}{l}\text { To determine the ACTISPP } \\
\text { sample characteristics. }\end{array}$ & $\begin{array}{l}\text { Comparison of an ACTISPP sample } \\
\text { of emergency department patients } \\
\text { with a criterion standard }\end{array}$ & $\begin{array}{l}\text { ACTSIPP sample (iii) } \\
\text { Sample (ii) }\end{array}$ & $\begin{array}{l}\text { Frequencies, cross- } \\
\text { tabulations and Chi- } \\
\text { square statistics }\end{array}$ \\
\hline $\begin{array}{l}\text { 7: The Health Consequences } \\
\text { of Injury Questionnaire }\end{array}$ & $\begin{array}{l}\text { To develop a quality-of-life } \\
\text { based injury outcome instrument } \\
\text { for use in large scale surveys }\end{array}$ & $\begin{array}{l}\text { Criterion validity study and a } \\
\text { test-retest reliability study }\end{array}$ & From sample (iii) & $\begin{array}{l}\text { Correlation coefficients } \\
\text { and measurements of } \\
\text { difference }\end{array}$ \\
\hline $\begin{array}{l}\text { 8: Health outcomes of injury } \\
\text { in the Australian Capital } \\
\text { Territory }\end{array}$ & $\begin{array}{l}\text { To quantity the outcomes of the } \\
\text { tull range of injuries occurring in } \\
\text { the Australian Capital Territory }\end{array}$ & $\begin{array}{l}\text { Prospective cohort study of injury } \\
\text { outcomes }\end{array}$ & From sample (iii) & $\begin{array}{l}\text { Frequencies and } \\
\text { cross tabulations }\end{array}$ \\
\hline $\begin{array}{l}\text { 9: Economic cost of minor } \\
\text { injury in the Australian Capital } \\
\text { Tenritory }\end{array}$ & $\begin{array}{l}\text { To estimate the incidence } \\
\text { based economic cost of } \\
\text { minor injury }\end{array}$ & 'Cost of illness' study & $\begin{array}{l}\text { Sample (i) } \\
\text { Sample (ii) }\end{array}$ & $\begin{array}{l}\text { Attaching costs from } \\
\text { AMTS(a), MBS(b) and } \\
\text { PBS(c) to incidence data }\end{array}$ \\
\hline 10: The Minor hiury Scale & $\begin{array}{l}\text { To develop a severity scale } \\
\text { for categorising minor injury } \\
\text { based on predicted outcomes }\end{array}$ & $\begin{array}{l}\text { Prospective cohort study } \\
\text { of injury outcomes }\end{array}$ & From sample (iii) & $\begin{array}{l}\text { Tabulations of outcomes } \\
\text { by injury type }\end{array}$ \\
\hline
\end{tabular}

a: AMTS = Australian Morbidity and Treatment Survey (Bridges-Webb et al. 1992)

b: MBS = Medicare Benefit Schedule (Commonwealth Department of Cornmunity Senvices and Hoalth 1990a)

c: PBS = Pharmaceutic Benefit Schedule (Commonwealth Department of Community Services and Health 19906) 


\section{Chapter Four}

\section{Injury and general practice in Australia}

\subsection{Introduction}

The injury literature suggests that in Australia, general practitioners (GPs) treat very few injuries (Lopez and Rennie 1969; Vimpani 1989; Nolan and Penny 1992). One consequence of this orthodox position is the neglect of general practice as a source of data for injury research. Because of the lack of injury research in general practice there is little evidence available to either support or refute the prevailing view. In an effort to clarify the position regarding general practice and injury in Australia, a secondary analysis was made of the only national population based injury data collected in Australia in the last decade. This chapter establishes the extent of the injury burden managed by general practice and describes the characteristics of those people who seek general practice attention rather than hospital emergency department services. The aim is to identify the role of general practitioners in managing injury in Australia.

\subsection{Methods}

\subsubsection{Overview of the National Health Survey 1989-90}

Unit record data was obtained from the National Health Survey 1989-90 (NHS) conducted by the Australian Bureau of Statistics (ABS 1992).

In the NHS, about one third of one per cent of the Australian population were selected using multi-stage (for private dwellings) and list (for non-private dwellings) sampling techniques and interviewed over a twelve month period October 1989 to September 1990. Excluded from the sample were non-Australian service personnel, overseas visitors, residents of hospitals, nursing homes, convalescent homes, boarding schools and military establishments. There were 56,803 people approached from an effective 
sample of 22,202 households. Adult members of the households were interviewed by trained $\mathrm{ABS}$ interviewers and, where relevant, asked to provide information about children in the household. Completed questionnaires were obtained from $96 \%$ of the subjects.

The interview process used health service contacts as a focus for data collection. Injuries were identified when they arose as reasons for health-related actions in the two weeks preceding interview. A further question relating to any other injury in the previous two weeks ensured the completeness of the data collected. All conditions that corresponded to those classified by ICD-9 from 800 to 995 were coded by the ABS to 049 irrespective of type or nature of injury. For each of up to eight medical conditions identified by each subject, they were asked whether they felt an accident caused the problem and, if so, when and where this accident (or, if more than one, their most recent accident) occurred.

Variables considered in this analysis include: whether injured, age, sex, country of birth, employment status, and whether presented to hospital or general practice for medical primary care. A variable 'reduced activity days' was created for each patient by summing the days reported for each of the mutually exclusive categories - days in hospital, days away from work/school and other days of reduced activity.

\subsubsection{Data analysis}

Data analysis was performed using the Statistics Package for the Social Sciences (SPSS Inc. 1990) and consisted, initially, of cross-tabulating within the sample to isolate specific subgroups of interest. Population estimates were calculated by weighting the sample by a weight variable supplied in the data-base. Standard errors were obtained from ABS tables (ABS 1992). Relationships were assessed using Chisquared and regression statistics on the unweighted data. Differences in the variable 'reduced activity days' between groups of patients were assessed using Chi-square statistics on the unweighted data. Regression analysis was performed, also on the 
unweighted data, to identify those characteristics of the injured person that might explain their choice of service provider. A logistic model was used for the regression analysis, with age, sex, country of birth, employment status and location of accident used as explanatory variables, and whether or not the patient was treated in general practice used as the outcome variable. Confidence limits of ratios were calculated according to standard methods (Cochran 1977).

\subsection{Results}

Of the 54,576 respondents, 4,137 were recorded as suffering from the effects of an injury during the two-week recall period. About $25 \%$ percent of these people had sought medical attention for the injury; $15 \%$ were treated by their GP only, $5 \%$ were treated in an emergency department only, $2 \%$ attended both an emergency department and general practice, $1.5 \%$ were treated by specialists only, $0.5 \%$ were treated by an emergency department and specialist and $0.5 \%$ were hospitalised (Table 4.1). After application of weights, in a full year there were an estimated 5,811,858 presentations to Australian general practices for treatment of an injury and this was more than twice the estimated presentations to hospital emergency departments.

Of the 4,137 subjects in the sample who were recorded as suffering from the effects of an injury during the two-week recall period there were 1,976 individuals whose relevant injury had been sustained in an accident that had occurred during this twoweek period. In this group of people who had sustained new injuries, about $16 \%$ attended general practice only, 7\% attended an emergency department only and 3\% attended both places of treatment. The ratio of general practice visits to hospital visits for first presentations after injury in the Australian population was 2.8:1 
Table 4.1 Estimated number and standard error (SE) of presentations to hospital and general practice in Australia for treatment of injury in any two week period during 1990-91.

\begin{tabular}{lcc}
\hline Place of presentation & No. of presentations & (SE) \\
\hline Emergency department only & 69,720 & $(5,400)$ \\
General practice only & 198,442 & $(7,500)$ \\
Specialist only & 22,142 & $(2,700)$ \\
Emergency department \& general practice & 25,091 & $(2,850)$ \\
Emergency department \& specialist & 4,048 & $(1,150)$ \\
Hospital admission & 7,176 & $(1,125)$ \\
\hline
\end{tabular}

Ratio of general practice to emergency department $=2.8: 1(95 \% \mathrm{Cl}$ 2.3-3.2)

The number of days of reduced activity during the relevant two week period that was due to the reported injury was used as a proxy for injury severity. There was a marked difference between the number of days of reduced activity of the injuries reported by those people who saw a doctor during the two week period and those who did not (Table 4.2). There was no significant difference, however, between the number of days of reduced activity resulting from injuries seen in general practices and those seen at the hospitals (Table 4.3).

Table 4.2 Distribution of subjects according to number of days of reduced activity due to injury by whether injured person sought medical attention.

\begin{tabular}{|c|c|c|c|c|c|c|c|}
\hline \multirow[b]{2}{*}{ Attended Doctor } & \multicolumn{6}{|c|}{ Reduced activity days } & \multirow[t]{2}{*}{ Totals } \\
\hline & 0 & 1 & 2 & $3-5$ & $6-10$ & $11-14$ & \\
\hline Yes & 499 & 86 & 69 & 107 & 81 & 192 & 1034 \\
\hline No & 2669 & 116 & 69 & 72 & 50 & 127 & 3103 \\
\hline Totals & 3168 & 202 & 138 & 179 & 131 & 319 & 4137 \\
\hline
\end{tabular}


Table 4.3 Distribution of subjects according to number of days of reduced activity due to injury by place of medical primary care.

\begin{tabular}{lccccccc}
\hline & \multicolumn{4}{c}{ Reduced activity days } & \multicolumn{2}{c}{ Totals } \\
\cline { 2 - 8 } Place of presentation & 0 & 1 & 2 & $3-5$ & $6-10$ & $11-14$ & \\
\hline Emergency department & 114 & 19 & 18 & 22 & 12 & 33 & 218 \\
General practice & 324 & 55 & 37 & 66 & 55 & 98 & 635 \\
\hline Totals & 438 & 74 & 55 & 88 & 67 & 131 & 853 \\
\hline$x^{2} p>0.05$ & & & & & & &
\end{tabular}

Although the severities of the injuries presenting to general practices and emergency departments did not differ, the two groups of injuries were different with respect to several characteristics of the injured people (Table 4.4). Patients presenting to general practice in the two weeks prior to interview tended to be older, non-Australian and non-European born, and more often employed than the group attending hospital emergency departments. There was no difference between the general practice and emergency department attenders with regard to sex.

The place of most recent accident for those people who presented to emergency departments was significantly different from those who presented to general practice (Table 4.5). In particular, $31 \%$ of all injuries seen in emergency departments occurred in places of sport and recreation while only $19 \%$ of those attending general practices reported this as the place of the most recent accident. 
Table 4.4 Logistic regression analysis of characteristics which distinguish the subjects who presented to general practice compared to an emergency department for treatment of an injury during the two weeks prior to interview.

\begin{tabular}{|c|c|c|c|}
\hline & Coefficient & Adjusted odds ratio\# & $(95 \% \mathrm{Cl})$ \\
\hline \multicolumn{4}{|l|}{ Age } \\
\hline$<45$ yrs & - & 1.0 & - \\
\hline$\geq 45 \mathrm{yrs}$ & $* 0.58$ & 1.78 & $(1.19-2.66)$ \\
\hline \multicolumn{4}{|l|}{ Sex } \\
\hline female & - & 1.0 & - \\
\hline male & -0.054 & 0.95 & $(0.68-1.32)$ \\
\hline \multicolumn{4}{|l|}{ Country of birth } \\
\hline Australian and European born & - & 1.0 & - \\
\hline other born & "1.54 & 4.65 & $(1.09-19.69)$ \\
\hline \multicolumn{4}{|l|}{ Employment status } \\
\hline unemployed & - & 1.0 & - \\
\hline employed & ${ }^{\star *} 1.15$ & 3.15 & $(2.05-6.45)$ \\
\hline \multicolumn{4}{|l|}{ Place of accident } \\
\hline place of sport and leisure & - & 1.0 & - \\
\hline other & ${ }^{\star *} 0.53$ & 1.70 & $(1.21-2.35)$ \\
\hline
\end{tabular}

\# adjusted for all other variables in the model

$\mathrm{Cl}$, Confidence Interval

** $p<0.01, \quad p<0.05$

Table 4.5 Distribution of subjects according to place of most recent accident by place of primary medical care.

\begin{tabular}{lrrrrrrr}
\hline & \multicolumn{4}{c}{ Place of most recent accident } & \multicolumn{2}{c}{ Totals } \\
\cline { 2 - 8 } Place of presentation & Work & Sport & Home & Road & School & Other \\
\hline Emergency department & 35 & 67 & 64 & 21 & 8 & 23 & 218 \\
General practice & 113 & 121 & 187 & 88 & 24 & 102 & 635 \\
\hline Totals & 148 & 188 & 251 & 109 & 32 & 21 & 853 \\
\hline$X^{2} p>0.05$ & & & & & & &
\end{tabular}




\subsection{Discussion}

The analysis of the NHS population-based injury data demonstrates the important part general practices play in the management of injury in our community. Both in the total number of injury treatments and in the number of new injury consultations, general practice attendances outnumber hospital presentations by more than 2 to 1 . It would seem that general practices are treating many more injury patients than is currently recognised.

As noted in Section 1.2.2, comparisons based upon total numbers of injuries in a given category can be misleading unless injury severity is taken into account. Robertson (1992) explains that because trivial injuries "which do not interfere with ones activities" are so common, they can distract attention from injuries with higher economic and health costs. The argument is relevant to this paper if the injuries which are being treated by GPs in such large numbers are, indeed, inconsequential injuries.

In this chapter, the number of days of reduced activity due to an injury was used as a measure of the injury's importance. The validity of using this measure of severity is supported by the results indicating a significant difference between the severity of those injuries presenting for medical attention and those injuries which did not. The fact that there was no significant difference between the severity of injuries presenting to general practice and those presenting to hospital, substantiates the importance of the GP role indicated by the total number of presentations. Injury research based exclusively upon hospital-treated patients would underestimate the public health impact of injury in our community.

Although the severity of the group of injuries attending general practice and hospital were similar there were other ways in which the two groups differed. The most striking difference was with respect to the age of the injured persons, but employment status, country of birth and place of accident also predicted place of presentation. The group of injuries which received primary care in general practice did not have the same characteristics as the group which attended hospitals. Injury research based upon 
hospital treated patients cannot necessarily be generalised to those injuries presenting to general practice.

General practitioners play a substantial part in the community's management of the injury problem. Not only do they treat more injuries than are treated by hospital departments but they treat a different population of injured people. Results of hospital studies should not be generalised to the community at large without further examination of the exact nature of the role played by general practices. General practice samples should be included in population-based studies of injury epidemiology. 


\section{Chapter Five}

\section{Incidence and distribution of injury in the Australian Capital Territory}

\subsection{Introduction}

Despite its importance as a public health problem it is not yet possible to present an adequate overview of injury in Australia. As discussed in Chapter Two, the information that is available is largely obtained from mortality and hospital morbidity records, with special purpose databases adding to our knowledge of trauma admissions, emergency department presentations, spinal injuries, occupational injuries and road traffic injury. In the area of injury and general practice, there is a particular lack of information. The epidemiology of injury receiving primary care in general practice has not yet been included in population estimates. As shown in chapter four, a substantial number of injuries do receive primary care in general practice. The current estimates of the magnitude of the injury problem in Australia therefore grossly underestimate the extent of the problem.

This chapter describes a study of the incidence and distribution of injury in a defined Australian population.

\subsection{Methods}

\subsubsection{Study design}

A descriptive incidence survey was conducted in the Australian Capital Territory (ACT) during 1992 to document the range of injury types and severities. Numerator data was obtained from centres of medical service provision within the ACT during the study period. Denominator and mortality data were obtained from the Australian Bureau of Statistics (ABS 1993). 


\subsubsection{Participants and process}

\section{The general practice sample}

For the purposes of this study, injury was defined as a medically-treated condition classifiable by an ICD-9 code between 800 and 995 . A general practitioner was defined as someone who considered him or herself to be engaged in general practice and who performed at least one, four-hour session of this work per week. Equating this workload to at least 1000 non-specialist items of service per year, the Australian Health Insurance Commission (HIC) identified 273 such practitioners in the ACT during 1992. Personal details of these practitioners were not available as privacy laws in Australia prevent the release of this information except under special circumstances with ministerial approval. It was not possible, therefore, to contact this sample.

Instead, the sample of GPs used in this study was drawn from a register of 220 ACT medical practitioners compiled by the New South Wales branch of the Royal Australian College of General Practitioners (RACGP). This list of ACT medical practitioners is continually amended and updated from a variety of sources and is not restricted to members of the RACGP.

Sample size calculations for the study were based upon an hypothesised injury rate of 224 per 1,000 people per annum (Gallagher et al. 1984), a GP to emergency department ratio of 2:1 (Chapter 4) a desired confidence of $95 \%$ and a supposition that each GP in the ACT would see 3.85 new injuries per week (Bridges-Webb et al. 1992). Anticipating a response rate of $85 \%$ from the sampled GPs, power calculations suggested that 39 GPs sampled for two weeks would provide estimates of GP attendances within $30 \%$ of the true value.

Thirty-nine medical practitioners were randomly selected from the RACGP list of ACT GPs. Attempts were then made to trace all selected practitioners to confirm whether or not they fulfilled the criteria of the GP definition used in the study and if so, to obtain accurate practice addresses. Those who did not fulfil the criteria were rejected. 
Those selected practitioners who were identified as GPs were approached on a sequential basis over a twelve month period and invited to participate in the study. Approaches were made first by an explanatory letter followed by a phone call with the GP and, where necessary, by a personal interview to discuss details. For two weeks after agreeing to participate, consenting GPs completed a short survey form (Appendix - 1) for each patient whom they considered to have presented with an acute injury. In order to ensure good quality data the GPs were visited on several occasions over the two week period to answer questions, collect forms, and encourage compliance.

The variables obtained from this process included the patient's age and sex, the nature of injury and body part injured, the mechanism of injury, whether the presentation was the first visit to a doctor for that injury, and whether the patient was admitted to hospital for treatment. Where two or more injuries were sustained by a single patient, only the most serious of the injuries was used in the analysis.

\section{The emergency department sample}

The hospital emergency department attendance registers were used as a sampling frame for the criterion sample. On every eighth day from 20 January 1992 to 19 January 1993, those people presenting with a complaint that could have resulted from an injury (ICD-9 800-995) were identified from the registers of both hospitals. These hospital medical records were drawn to confirm compliance with the case definition. This method of identifying injuries presenting to emergency departments is similar to that used in previous injury incidence studies (Gallagher et al 1984) and has been shown to be a highly-effective means of identifying cases (Kalsbeek et al 1992). All firstpresentation new injuries in adult residents of the ACT attending the emergency department on these days were included in the incidence sample. A one day in eight sample was chosen so as to cover all weeks in the study period with each day of the week being equally represented. This was necessary because of the well-recognised cyclical patterns in the presentation of injury. On the basis of about 24,000 injured 
people presenting to the emergency departments per year it was estimated that the selection strategy would ensure that the sample contained enough occurrences of those injuries which occurred as rarely as one in 20 injuries in order to be $95 \%$ sure of predicting their incidence to within one percent of the true value.

The effectiveness of the register screen was retrospectively checked by randomly selecting $10 \%$ of the sample days and drawing the medical records for all people presenting on that day, whether or not their presenting complaint was related to injury. The number of presenting injuries that had originally been missed on these days was noted.

Data were extracted from hospital records. The subjects age, sex and postcode of residence and whether the patient was admitted to hospital were routinely documented in the medical records by hospital clerical staff. The nature of injury and body part injured were routinely documented by medical staff. The author and two trained assistants coded the medical records into Injury Surveillance Information System (ISIS) codes, (National Injury Surveillance Unit 1992) and entered it into the SPSS statistical package (SPSS inc. 1990) for analysis. The injury details were converted to AIS codes using a conversion programme written in SPSS by the author, a trained AIS coder. Where two or more injuries were sustained by a single patient, only the most serious of the injuries was used in the analysis.

\section{Mortality count}

The total number of deaths occurring in the ACT from injury (ICD-9 800-995) during 1992 was obtained from the ABS (1992). Crude death rates were obtained by dividing the number of deaths in the 12 month period by the number of people in the study population. 


\subsubsection{Analysis}

The emergency department sample was used to estimate the total number of ACT residents presenting to emergency departments in the ACT for treatment of a new injury over the study period. This was achieved by first calculating a mean and standard error for the number of injuries presenting per day and multiplying by the number of days in the sample period to estimate the size of the total population.

The mean number of injuries per GP week and standard deviation were calculated from the data obtained from the sample of GPs. An estimate of the total number of injuries in the ACT that received primary care in general practice during 1992 was calculated by multiplying the sample mean by the number of weeks in the year and the number of GPs in the ACT.

\subsection{Results}

Of the 39 general practitioners selected from the RACGP list, 13 were found to have left the ACT or to have retired from practice or to be otherwise not a GP within the definitions of the study. One GP refused to participate and one withdrew from the study after agreeing to be involved. Thus 24 of the eligible 26 GPs sampled participated in the study, representing a response rate of $90 \%$. Fifteen GPs were male and 9 female. Six worked in Government Health Centres, six as solo practitioners, four at extended hours medical centres, one at a university health service and the rest in group practices. There was no profile of GPs in the ACT available for comparison purposes. Forty-six weeks of the 12 month study period were covered by at least one of the participating GPs. Details of 79 new injuries were obtained, with a mean of 1.7 injuries per GP week.

There were 3008 injuries recorded in the hospital sample. The medical records of patients identified from the register were not available in five percent of cases and the remainder provided a satisfactory account of the required variables. The search of the 
emergency department register was found to be an effective method of identifying injury presentations to the emergency department. On the retrospective check of $10 \%$ of days, less than one percent of injury presentations were found to have been missed.

When the ABS, general practice and emergency department data were combined, estimates suggest 48,500 (95\% CI 41,000 - 56,000) injuries requiring medical attention were sustained by ACT residents in the ACT from January 1992 to January 1993. This represents 164 (95\% CI 139 - 189) injuries per 1,000 inhabitants per year. General practice was the first place of presentation for primary care in approximately one half of cases (Table 5.1). Thus the ratio of deaths : admissions : emergency department presentations : general practice attendances was $1: 28: 596: 592$.

Table 5.1 Estimated number of injuries in the ACT in 1992 by category of injury

\begin{tabular}{lrrr}
\hline Category of injury & Number & $(95 \%$ Cl for estimates $)$ \\
\hline Deaths & 40 & & \\
Hospital admissions & 1,120 & $(1,080-1,160)$ \\
Emergency department visits & 24,000 & $(23,000-25,000)$ \\
General practice attendances & 23,500 & $(17,000-30,000)$ \\
\hline
\end{tabular}

The emergency department and general practice data were combined to produce the injury distribution pattern illustrated in Figure 5.1. Injury rates were highest between the ages of 15 and 24 and over the age of 65 years. Injuries to males predominated in the early years while injuries to women were more frequent in the older age groups 
Figure 5.1 Injury rate in the ACT by age and sex

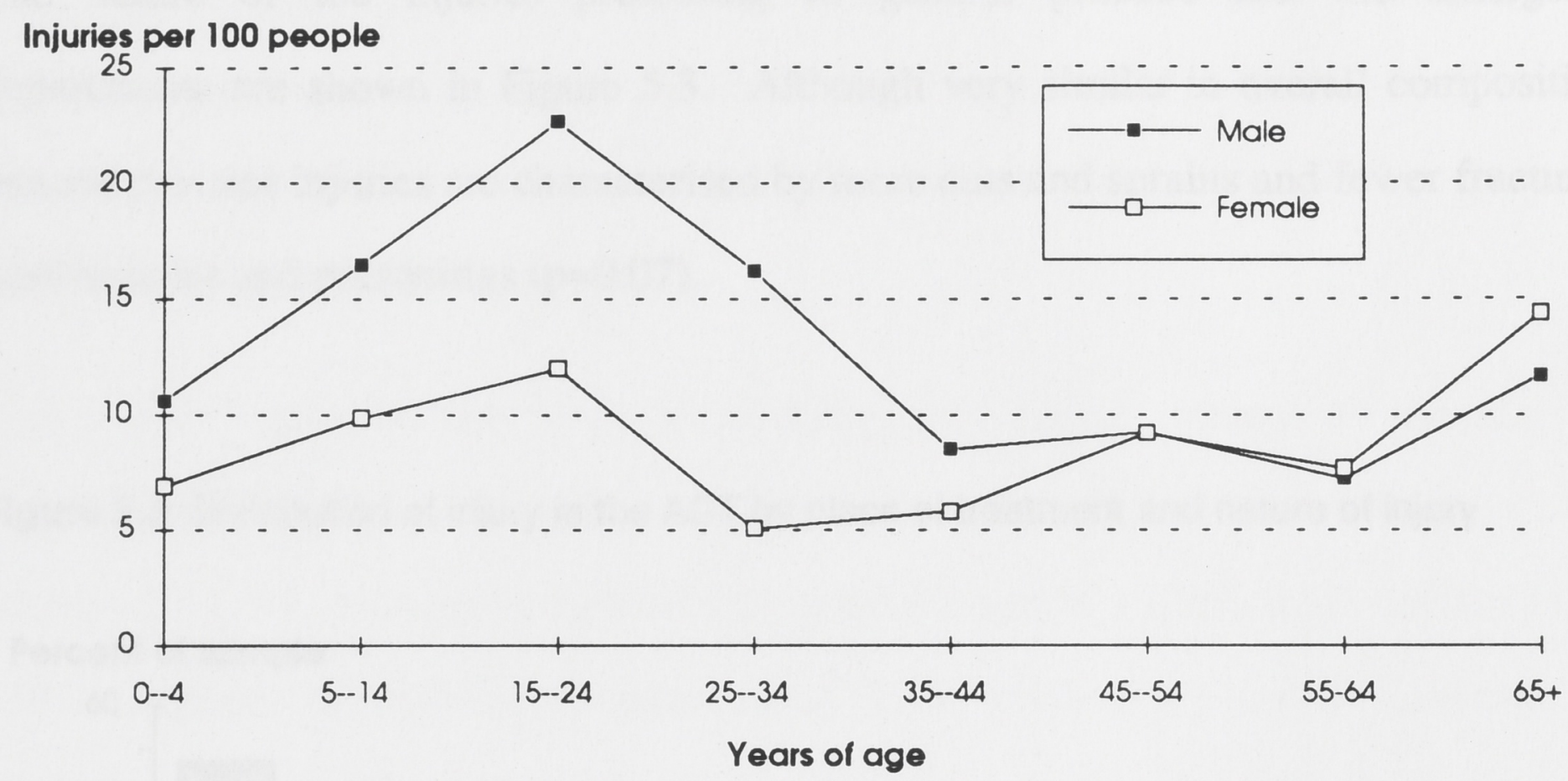

There were some differences in the age and sex distribution of patients presenting to emergency departments and general practices. The ratio of male to female injuries in the emergency department sample was $1.8: 1$ while in the general practice sample it was 1.5:1 $(\mathrm{p}=0.07)$. Injured patients attending general practice for treatment tended to be older than those presenting to hospital $(\mathrm{p}<0.001)$.

The profiles of the two samples by body-part injured were similar, however trunk injuries were shown to be a feature injuries presenting to GPs with relatively fewer head, face, upper limb and internal injuries $(\mathrm{p}<0.001)$ (Figure 5.2).

Figure 5.2 Distribution of injuries in the ACT by place of treatment and body-part injured

\section{Percent of sample}

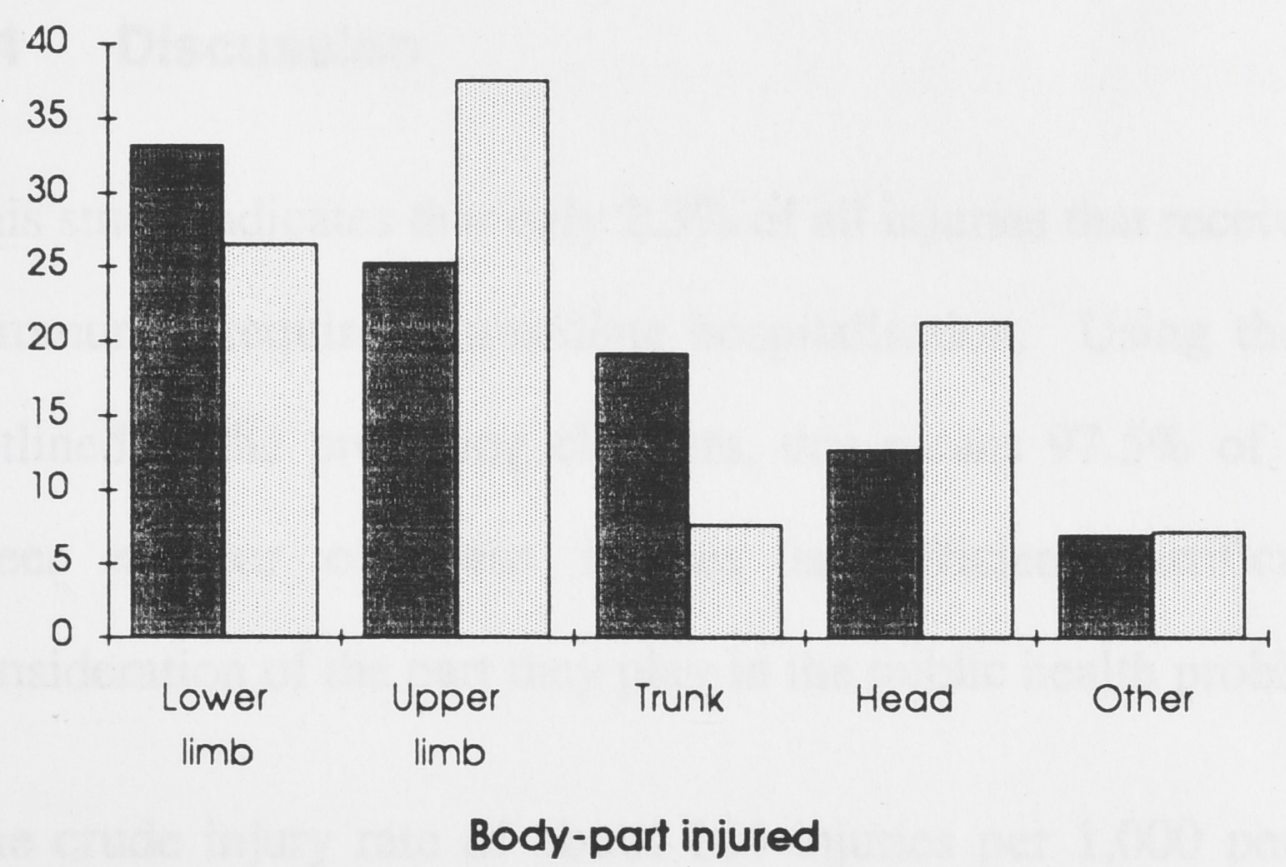

GP sample, $n=79$

ED sample, $n=3008$

Body-part injured 
The nature of the injuries presenting to general practice and the emergency departments are shown in Figure 5.3. Although very similar in overall composition, general practice injuries are characterised by more cuts and sprains and fewer fractures, head injuries and poisonings $(\mathrm{p}=0.07)$.

Figure 5.3 Distribution of injury in the ACT by place of treatment and nature of injury

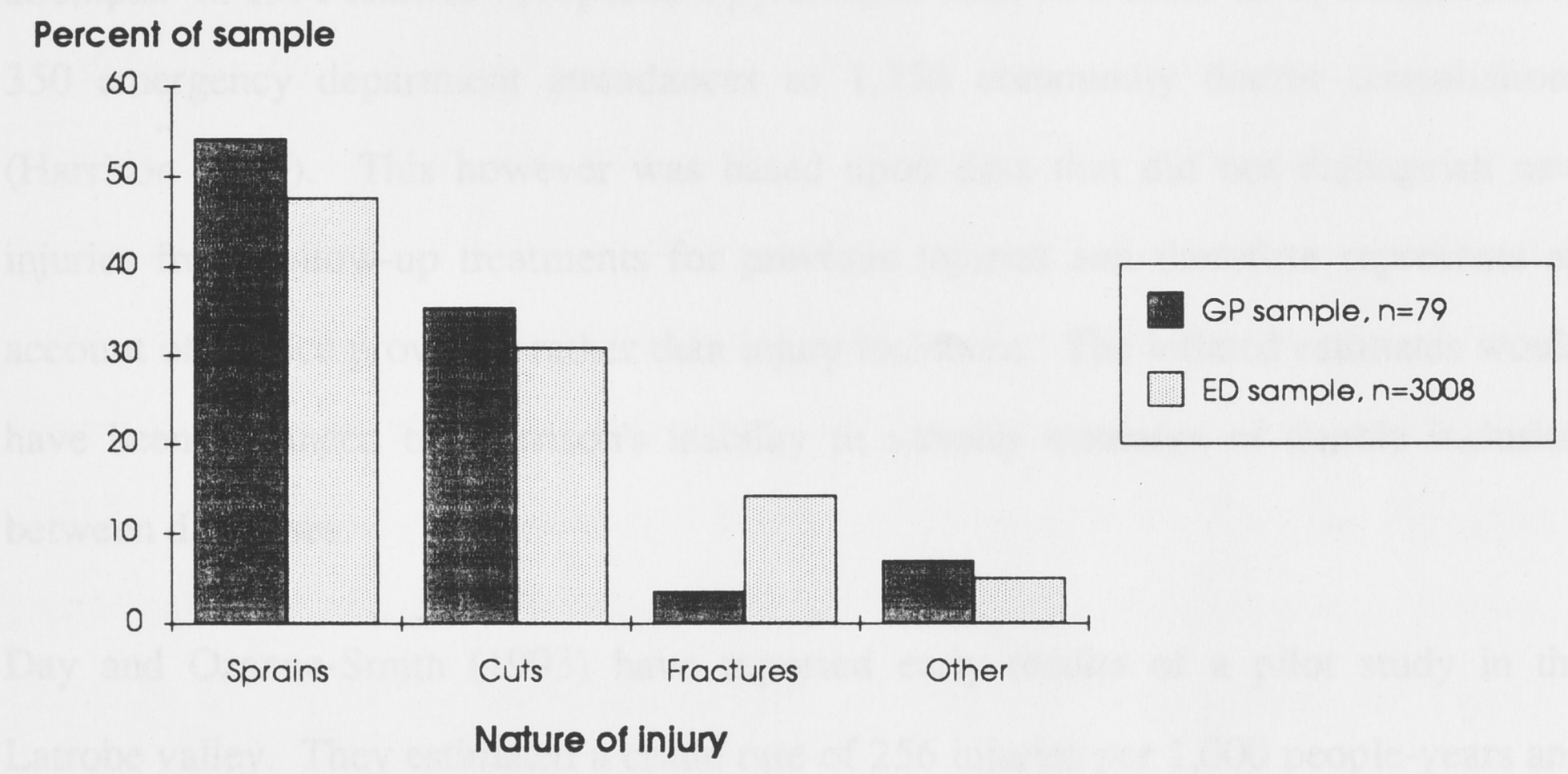

Admission rates for the two samples differed, with $5 \%$ of emergency department sample being admitted compared to none of those in the GP sample $(\mathrm{p}<0.001)$.

\subsection{Discussion}

This study indicates that only $2.5 \%$ of all injuries that received medical treatment in this community required immediate hospitalisation. Using the definition of minor injury outlined in the preceding chapters, this means $97.5 \%$ of all injuries are minor. The sheer number of these injuries is sufficient justification for further detailed consideration of the part they play in the public health problem of injury.

The crude injury rate of about 164 injuries per 1,000 person years presented in this study with a deaths : admissions : ambulatory care ratio of $1: 28: 1194$ is consistent 
with the other population-based studies of injury incidence introduced in Chapter Two. The US study identified an injury rate of 197 injuries per 1,000 person-years (Fife et al. 1984) while the rates in Scandinavia were 114 (Sahlin et al. 1990) and 119 (Linqvist 1989) injuries per 1000 person-years.

This study represents the most methodologically sound attempt to identify the dimensions of the injury incidence iceberg in Australia. There have been two other attempts. In 1991 Harrison proposed a pyramid of rates of 1 death to 40 admissions to 350 emergency department attendances to 1,350 community doctor consultations (Harrison 1991). This however was based upon data that did not distinguish new injuries from follow-up treatments for previous injuries and therefore represents an account of service provision rather than injury incidence. The inflated estimates would have been enhanced by Harrison's inability to identify instances of double inclusion between databases.

Day and Ozanne-Smith (1993) have reported early results of a pilot study in the Latrobe valley. They estimated a crude rate of 256 injuries per 1,000 people-years and a ratio of hospital admissions to emergency department attendances to general practice attendances of 1:5:10. The admission rate of $6 \%$ of all injuries is higher than might be expected from the literature and may have resulted from using hospital separation data to obtain rates, rather than incidence data, but the importance of the GP component of injury management is clearly illustrated.

The validity of the results presented depends to a large extent on the lack of bias in the study sample and an accurately-determined population at risk. With respect to these aspects, the Australian Capital Territory is particularly suitable for population studies of injury (Pearn and Nixon 1981). Obtaining accurate numerator and denominator 
values for injury rates is facilitated by the identifiable nature of Canberra's medical service providers, the completeness of the services offered, the geographically circumscribed region in which the injuries occur and the well-defined characteristics of the resident population.

In the hospital component of the study, identifying a sampling frame was a simple process and some confidence can be placed upon the representative nature of the survey findings. The GP list was more problematic. In a recent paper, the various possible lists for GP samples were reviewed and none of them were found to be without flaws (Saltman and Man 1992). Certainly, it would appear from the discrepancy between the numbers on the RACGP and HIC lists used in this study that some bias has been introduced into the GP sample. The study has managed to avoid, however, the response rate bias that is common in GP research (Bridges-Webb et al. 1992). No attempt was made to validate the responses supplied by the general practitioners. This omission was necessary as ethical considerations prevented a thorough search of the participating GP's records. This acceptance of the GPs' accounts of their service provision at face value is consistent with current practice (Bridges-Webb et al. 1992).

The results presented in the general practice component of this chapter are consistent with the findings of other Australian general practice studies once the differing methodologies are taken into consideration. In this paper, we have estimated that each GP sees 1.7 injuries per week. A pilot study conducted among GPs in the Shire of Bulla in 1992 reported 2.85 new injuries per GP-week (Ozanne-Smith and Brumen 1992). A population-based study of childhood injuries in Port Lincoln (Taylor and Cockington, 1990, personal communication) found 1.2 injuries per GP week. A childhood injury survey conducted at "one of the busiest community health centres" in Melbourne reported in 1992 found only "a few trivial cases per week" (Nolan and Penny 1992) and secondary analysis of data collected for the Australian Morbidity and Treatment Survey 1990-91 (AMTS) (Bridges-Webb et al. 1992) suggest that Canberra GPs treat 3.85 new injuries per week. 
The estimates of the Melbourne and AMTS studies are furthest from the Canberra result. However, the estimate from the Melbourne study is particularly unstable as only one practice was sampled and the AMTS study probably overstated the injury incidence because of difficulty in defining 'new cases'. In the Port Lincoln study and the study presented here, special attempts were made to identify new cases with the patients themselves identifying their injury as not having previously been presented to a doctor for treatment. The AMTS, on the other hand, was not designed to identify disease incidence and nor did it specifically address the problem of injury. In that study decisions on injury status were made by the medical practitioner, and as in many instances the recorded treatment given to these "new" cases in the AMTS was obviously follow-up management (eg removing sutures, trimming casts) these decisions may have been flawed.

Further limitations of the AMTS due to its lack of focus on injury are the lack of information about the injury event, the lack of contemporaneous hospital data, and the lack of details about the injury itself. Although GP selection was random, only a $50 \%$ response rate was obtained and no effort was made to validate directly the information provided by the GPs. Some of these problems were overcome in the Bulla Shire study but, as it was only a pilot for a more elaborate work to follow, sampling was not random and the data collection time was too restricted to take into account the seasonal variations in injury. Other explanations for variations in the findings of the studies include differences in the age structures of the study populations and differences in the definition of a general practitioner. Where variation in injury estimates cannot be explained by methodological differences, they might simply reflect regional differences in the numbers of GPs in the district and the structure of the local health care system.

The results of this study suggest that approximately half the injuries occurring in the $\mathrm{ACT}$ receive primary care in general practice. This compares to a ratio of $2.8: 1$ for 
new injuries found in the study described in Chapter Four. There are two plausible explanations for this difference. Probably the most important factor is a sociodemographic. The higher rate of GP attendance was found in the national sample which covered large areas of rural Australia where access to emergency department care is limited. Similarly this national sample also included those areas of suburban and inner-city Australia where general practitioners may provide more timely care than the crowded hospital system. In contradistinction, the ACT contains a mostly urban community of living in a planned city with excellent access to well-staffed emergency departments. Many of the general practices in the ACT are not equipped to treat injuries. In these conditions injured patients may have preferentially presented to the hospitals for treatment.

A second possible explanation for the lower ACT rates is a differential information bias between the two studies. Participating doctors in the ACT study may have neglected to record some of the injuries they treated during the course of their sampled fortnight. This would have resulted in an underestimate of the total number of injuries treated by GPs in the ACT and a falsely low GP to emergency department ratio.

A consequence of a lower-than-expected injury rate per GP in the ACT study was that the total number of injuries in the study was lower than anticipated. In turn this led to wide confidence intervals around the estimates of injury in general practice. Nevertheless even the minimum incidence estimate identified in this chapter supports the conclusion, established in Chapter Four, that a substantial number of injuries are being treated in general practice.

The characteristics of people presenting to general practice for treatment of an injury differ from those who present to hospital emergency departments. Differences also exist in the types of injuries treated. The general notion that emergency department injuries are more severe than those treated by GPs seems to be appropriate, as the study shows the latter group includes fewer fractures and fewer admissions. The differences that have been demonstrated between the hospital and general practice 
samples suggest that one cannot simply rely on hospital-based surveillance to obtain a complete picture of community injury.

Despite the limitations of this study that have been discussed, there is no question that minor injuries constitute the majority of the total number of injuries in the community. Quantification of the outcomes of these minor injuries must therefore be included in any attempt to document the full public health impact of injury in a community. 


\section{Chapter Six}

\section{The Australian Capital Territory Injury Surveillance and Prevention Project}

\subsection{Introduction}

Samples for the prospective follow-up study of injury outcomes, described in Chapter Eight, were drawn from the Australian Capital Territory Injury Surveillance and Prevention Project (ACTISPP). In this chapter, the ACTISPP database is evaluated as a means of identifying a representative sample of injured ACT residents.

\subsection{Methods}

\subsubsection{Study design}

A retrospective descriptive study of injuries treated in emergency departments of the ACT was conducted using information obtained from hospital records. The characteristics of the injury problem thus identified were compared with the surveillance system data for the sample population over the same time period. The ACTISPP database was evaluated in terms of its 'sensitivity' (ie: the measure of a system's ability to identify all of the true cases of injury in the surveyed population Graitcer 1987) and its representativeness (ie the extent to which the injured people detected by the surveillance system are typical of those people in the target population).

\subsubsection{Study sample (criterion sample)}

The study sample used as the criterion standard against which ACTISPP was compared was a subset of the emergency departments incidence sample described in detail in Chapter Five. The subset used in this study was all those subjects registered 
between 20th January 1992 and 19th September 1992. These time periods were chosen to coincide with the study dates for injuries registered in the ACTISPP project database during 1992.

\subsubsection{ACTISPP project database}

Between 20th January 1992 and 19th September 1992, ACTISPP collected data from people attending emergency departments for treatment of their injuries. The system was managed by the state government and concentrated upon identifying:

- the location where the injuries were occurring,

- the activities in which people were engaged at the time of the injury event,

- the way in which the injuries occurred,

- the factors involved in the injury event,

- the character of the injuries sustained and,

- the type of immediate emergency department care.

The surveillance data were collected by means of a questionnaire (Appendix - 2) completed at the time of presentation to the emergency department by both the injured person and the treating doctor. It was the responsibility of the emergency department staff to ensure that each patient who attended the hospital for first treatment of an injury was given a questionnaire. The patient was asked to complete the form while waiting for treatment. In the case of children, the information sheet was completed by the parent or guardian and for those patients whose injury prevented their being able to respond, accompanying friends or relatives were invited to assist. After the form was distributed no further attempt was made to encourage compliance and no record was kept of those patients to whom the form was given. Signed consent for further follow up was obtained at the time of the completion of the questionnaire. The patients were asked to return the questionnaire to their treating medical officer who recorded clinical 
details of the injury and what management had been arranged. The completed forms were returned to a central point for collection by the ACTISPP project officer three times a week.

\subsubsection{Data analysis}

The study sample was used to estimate the total number of ACT residents presenting to emergency departments in the ACT for treatment of a new injury over the study period. This was achieved by first calculating a mean and standard error for the number of injuries presenting per day and multiplying by the number of days in the sample period to estimate the size of the total population. The sensitivity of the surveillance system was obtained by expressing the number of injuries identified by ACTISPP as a percentage of the estimated total number of injuries in the target population. Confidence intervals of this sensitivity estimate were obtained by dividing the number of injuries recorded in ACTISPP by the $95 \%$ limits of the population estimate.

The representativeness of ACTISPP was assessed by identifying those injuries in the ACTISPP database that had occurred to ACT residents between 20th January 1992 and 19th September 1992 and comparing them with the study sample for age, sex, postcode of residence, nature of injury, body-part injured and whether the patient was hospitalised. The study sample was considered to represent the true distribution of the variables in the population and where the ACTISPP database differed from this criterion sample it was considered to be non-representative. Percentage differences for each of the categories of the study variables were identified and the statistical significance for each of these differences was assessed using Chi-squared statistics with an alpha value of 0.05 . With the criterion sample size set large enough to identify the population injury distribution accurately, there was sufficient power in the study to identify statistical significance for two percent differences from ACTISPP with a power of $80 \%$. 


\subsection{Results}

\subsubsection{Sensitivity}

There were 2,013 subjects in the study sample between the dates of this study. Thus $16,100(95 \%$ CI 15,500 - 16,700) first-presentation injuries presented to emergency departments in the ACT during the study period. ACTISPP registered 4,971 people during this same time resulting in a sensitivity for the surveillance system of $31 \%(95 \%$ CI $30 \%-32 \%)$.

\subsubsection{Representativeness}

The ratio of male to females in the ACTISPP data base and the criterion sample were 1.8:1 and $1.9: 1$ respectively $(\mathrm{p}=0.234)$.

The age distributions of the two samples were similar except that the 5-14 year age group was over-represented in the ACTISPP database $(\mathrm{p}<0.001)$ (Figure 6.1).

Figure 6.1 Distribution of age in ACTISPP database and criterion sample

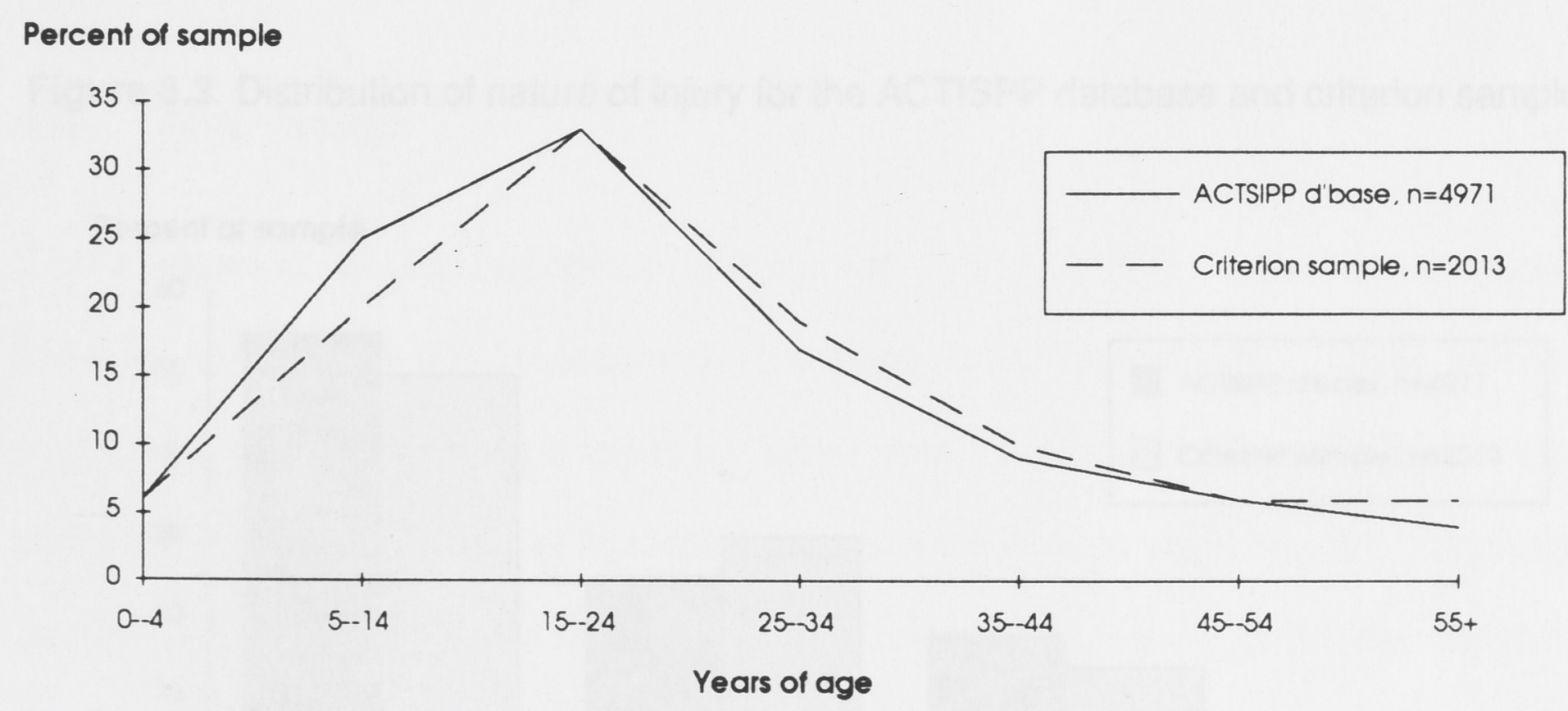

There were no statistically significant differences between the ACTISPP database and the criterion standard by postcode of residence (Figure 6.2). As far as postcode of 
residence is a valid measure of socio-economic status, there was no apparent SES bias in the surveillance process.

Figure 6.2 Distribution of postcode of residence for the ACTISPP database and criterion sample

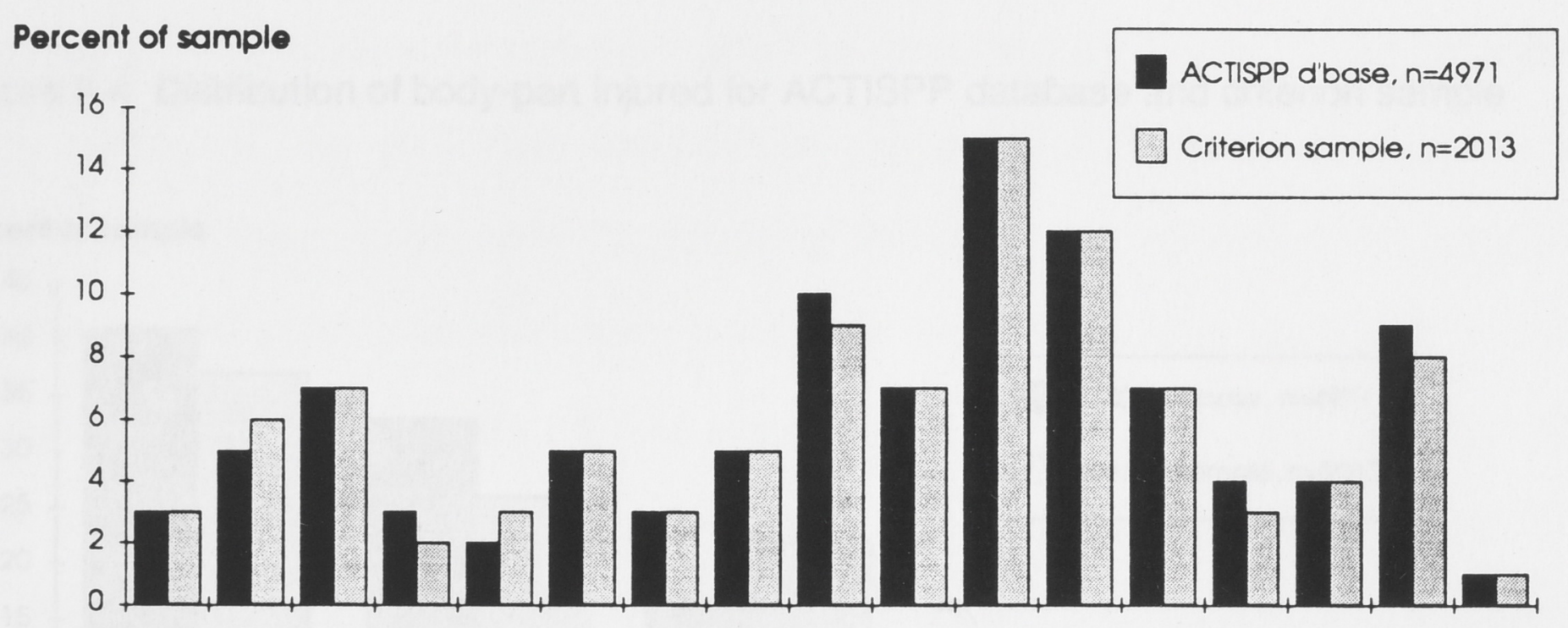

Postcode of residence

There were some differences in the distribution of injury types in ACTISPP data and the target population $(\mathrm{p}<0.001)$ (Figure 6.3). This difference was less than five percent in any category and did not markedly affect the surveillance data profile for this variable.

Figure 6.3 Distribution of nature of injury for the ACTISPP database and criterion sample

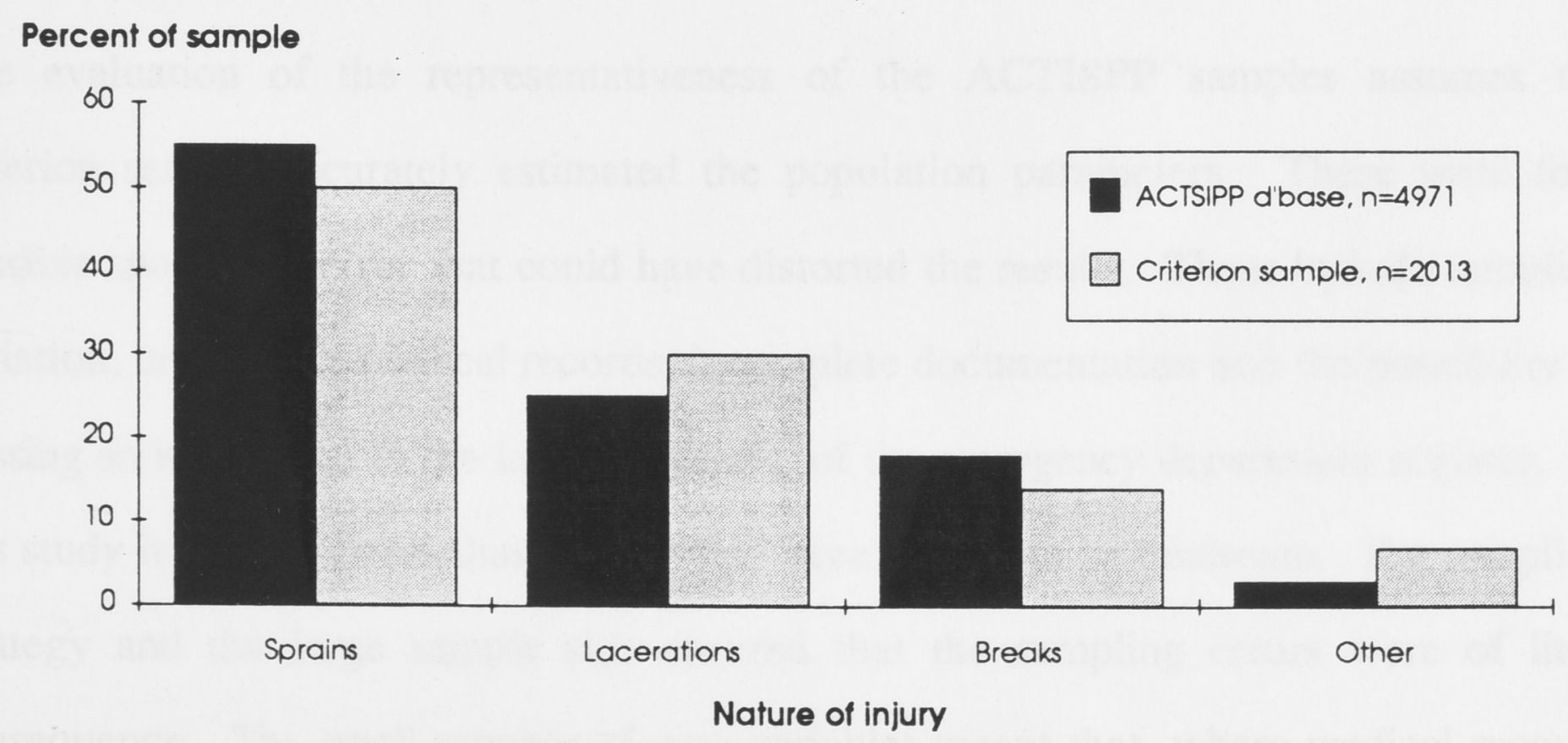


The ACTISPP sample differed from the criterion standard for body part injured with lower limb injuries being $7 \%$ more common in the ACTISPP database $(p<0.001)$. Again, however, the overall pattern of the ACTISPP data followed the expected profile (Figure 6.4).

Figure 6.4 Distribution of body-part injured for ACTISPP database and criterion sample

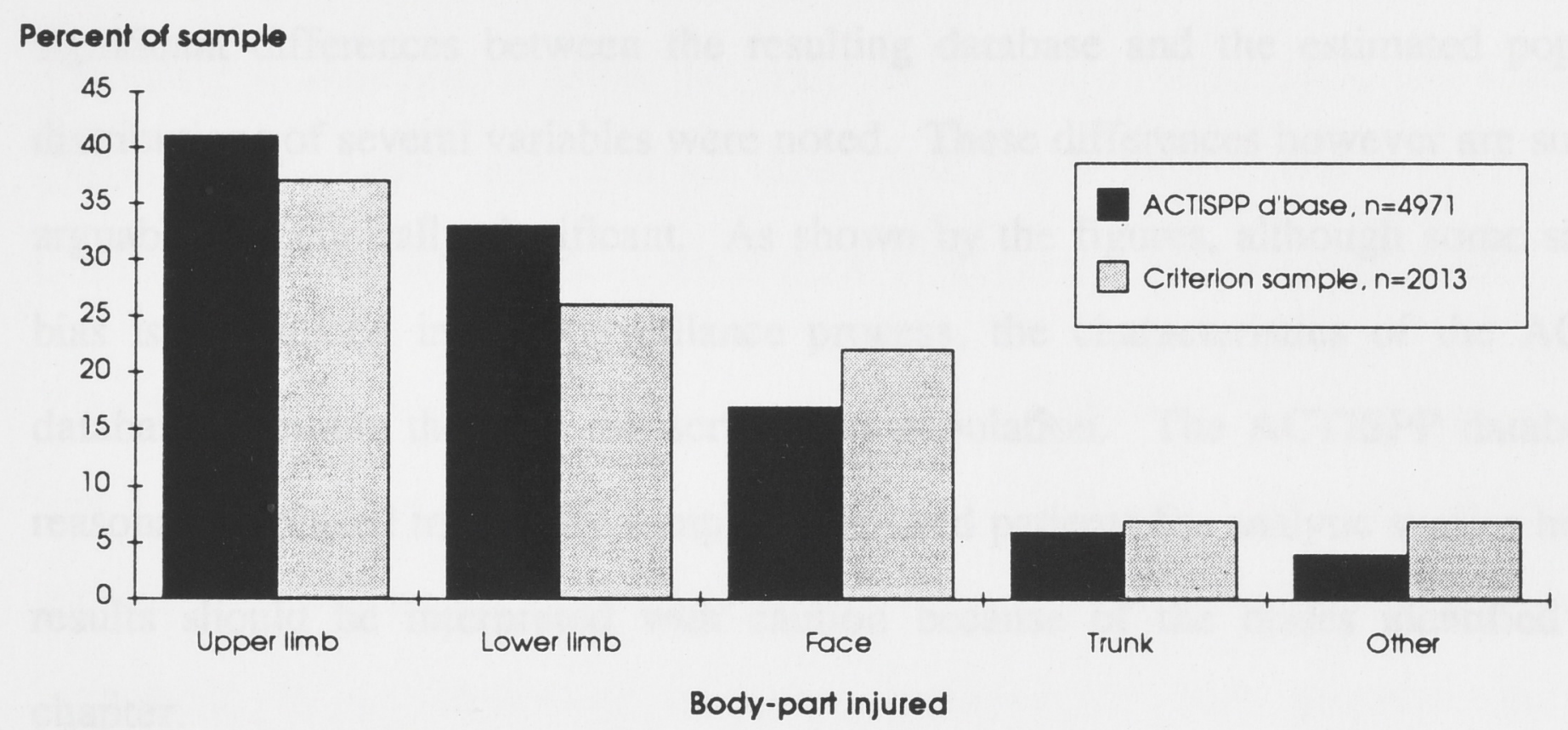

About two percent of the ACTISPP sample were admitted to hospital compared with an estimated four percent in the target population $(\mathrm{p}<0.001)$.

\subsection{Discussion}

The evaluation of the representativeness of the ACTISPP samples assumes the criterion sample accurately estimated the population parameters. There were four possible sources of error that could have distorted the results. These include sampling variation, unavailable medical records, incomplete documentation and the possibility of missing an injury case in the initial screening of the emergency department register. In this study it would appear that these errors have been kept to minimum. The sampling strategy and the large sample size ensured that the sampling errors were of little consequence. The small number of core variables meant that, where medical records were available, complete data was obtained. That five percent of the medical records 
were missing, however, was clearly a problem and a relevant bias may have been introduced at this point.

This study has examined the representativeness and sensitivity of the ACTISPP database by comparing it with the estimated frequency and distribution of injury in the population. Approximately one-third of all incident injuries treated in the emergency departments of the ACT are included in the ACTISPP database. Statistically significant differences between the resulting database and the estimated population distributions of several variables were noted. These differences however are small and arguably not clinically significant. As shown by the figures, although some sampling bias is introduced in the surveillance process, the characteristics of the ACTISPP database resemble those of the scrutinised population. The ACTISPP database can reasonably be used to identify samples of injured patients for analytic studies however, results should be interpreted with caution because of the biases identified in this chapter. 


\section{Chapter Seven}

\section{The Health Consequences of Injury Questionnaire}

\subsection{Introduction}

Injury research has long been hampered by the lack of a survey instrument capable of measuring, in dimensional units, the health consequences of all types and severities of injury. This chapter describes an instrument, the Health Consequences of Injury Questionnaire (HCIQ), for use in epidemiological studies of injury outcomes.

The starting point for the development of the new instrument was the Quality of WellBeing scale described in Section 2.4.3; a quality-of-life, general health status measure that in many respects is ideally suited to the needs of injury epidemiologists. The QWB instrument is an extremely complex questionnaire that requires interviewer administration, takes approximately 40 minutes to complete and is therefore of limited use in large scale surveys. However, the developers of the scale have suggested that it is possible to estimate the QWB scores for health profiles constructed from a different instrument if the items used are "similar in many respects to the QWB items" (Kaplan et al. 1989b). They report an attempt to map the QWB values onto data collected from the National Health Interview Survey (NHIS) in the United States of America (Erickson et al. 1989): In that instance, the mapping was achieved by identifying the specific items in the NHIS that appeared to be assessing the same concepts of healthrelated quality of life that were assessed by the QWB instrument, and then attaching the appropriate QWB weights to the relevant NHIS items.

Using the same principle, the Health Consequences of Injury Questionnaire (HCIQ) was constructed with items "similar in many respects" to the QWB instrument but arranged in a format amenable to rapid self-completion. The aim in this chapter is to evaluate the HCIQ in terms of its capacity to predict QWB scores and in terms of its 
reliability and practicability as a survey instrument for epidemiological studies of injury outcomes.

\subsection{Methods}

\subsubsection{Study design}

Two prospective surveys of injured adults were conducted to evaluate the criterion validity, the reliability and the practicability of the HCIQ. The validity study involved a comparison between the results obtained from the HCIQ with those from a concurrently-administered criterion standard - the QWB scale. The second study involved a test-retest administration of the HCIQ with the comparability of the results of the two administrations being used as a measure of its reliability.

\subsubsection{Participants}

The subjects selected in the study samples were identified from the Australian Capital Territory Injury Surveillance and Prevention Project (ACTISPP) database. Those subjects included in the criterion validity sample for this paper were all those people, 16 years of age or older, who registered in the ACTISPP survey between 5 September 1991 and 30 September 1991, who had a condition classifiable by ICD-9 codes 800995 and who, at the time of their presentation to the emergency department, signed a consent indicating their willingness to participate in further follow-up. The reliability sample included all those injured and consenting adults who registered in the ACTISPP survey between September 13, 1992 and October 25, 1992. The duration of the respective study periods was chosen in anticipation of obtaining approximately 100 subjects in each sample. This sample size was necessary to ensure a $95 \%$ chance of obtaining correlation-coefficient estimates within $20 \%$ of the true value. 


\subsubsection{Survey instruments and process}

The QWB instrument is essentially a functional status measure combined with a symptom check-list (Section 2.4.1). It records a subject's health profile and ascribes weights (QWB weights) to the components of the profile to convert the identified health status to a unitary index (Appendix-3). The index ranges from zero to one where one represents full health and zero represents death. QWB weights were derived from community surveys in the United States and represent the social preference, or undesirability value of the various health states ascertained by the QWB instrument. Thus, the QWB scale enables a subject's health profile to be both described and quantified. It includes all possible health states from full health to death on its scale. The index has been widely used and rigorously evaluated. As a general health index, it has been shown to be valid, reliable and sensitive to change of health states over time (Section 2.4.1). The QWB scale has also been used to measure the consequences of major trauma (Holbrook et al. 1994) where comparisons with the Functional Disability Score (MacKenzie et al. 1986) highlighted the former as a useful measure of injury outcomes.

The HCIQ records the symptoms experienced by the subject at any time during the preceding week that can be attributed to a given injury. It also records any functional limitations the injury produces with respect to the subject's mobility and physical and social activity during this period. The HCIQ focuses on the effects of injury, in contrast to the QWB scale which is a general health measure. The HCIQ also differs from the QWB scale in that it assesses the 'worst state' experienced by the subject over the preceding week, rather than the average health status during that same period. The most deleterious effect on health and functional status from injury typically occurs at the time of the event, after which a gradual recovery takes place. In averaging a recovering patient's health state over six days, the QWB scale under-estimates the maximum health loss suffered by the patient during the period. For example, concussed patients may be unconscious and hospitalised in their first day after the injury event, yet, because they recover completely by the second or third day, the 
QWB scale assigns these patients a trivial health loss score. The HCIQ may circumvent this problem by identifying the greatest limitation experienced by the subject in each of the dimensions over the preceding week, thus creating a 'worst state' profile to which QWB weights can then be attached. This 'worst state' score is then attributed to the first day of the period covered.

There are two forms of the HCIQ. The first instructs subjects to recall limitations experienced in the week since their injury and is designed to be administered one week after the injury event (Appendix - 4). The second version is used for the follow-up administrations of the questionnaire in prospective outcome studies and asks for limitations experienced during the week preceding the repeat completion (Appendix 5). It was the first version that was used in the studies described below.

\section{The criterion validity study}

The age, sex, postcode of residence, nature of injury and body part injured were obtained from the ACTISPP database for those people registering as newly-injured during the study period. Four to six days after their injury, the selected subjects were contacted by telephone and personal, face-to-face interviews were arranged where the subjects completed both the QWB instrument and the HCIQ. The timing of these interviews to fall between four and six days after the injury occurrence was necessary to ensure that the QWB instrument was administered according to its standard protocol while at the same time producing a score that reflected the health outcomes of the relevant injury. Any of the selected sample who were unable to be contacted or who were unavailable for interview during this two day period were recorded as nonresponders, as were those people who declined the interview. Patients who had been admitted to hospital for immediate treatment of their injury were not specifically excluded from the survey although the nature of this follow-up process made them less likely to be included. 
There were four research assistants who conducted the interviews. These research assistants underwent a complete training session as a group using the manual and audio cassette tapes supplied by the authors of the QWB scale. This session was repeated after each had conducted their first few interviews to resolve any issues that had arisen. At each subject interview, the research assistants administered the QWB instrument and also gave the subjects the HCIQ to complete without assistance. For half of the respondents the QWB instrument was administered first, followed by the HCIQ, and to the rest, the QWB instrument was administered second.

\section{The reliability study}

To a second sample of subjects, the HCIQ was mailed during the first week after their injury event. On the instrument's return, the HCIQ was sent out again for the subject to complete for the second time. Those subjects who did not return their HCIQ questionnaires within two weeks of having been sent a copy were mailed a reminder, and a second reminder two weeks later if the first did not produce a response. Within the eight week period over which the study was conducted there was considerable variation between the subjects completion spacings, but no subject had less than one week test-retest lag period.

\subsubsection{Measurement and data analysis}

The QWB weights were used to score both the QWB instrument and the HCIQ. The main outcome measure was the QWB score as derived from the QWB instrument and as estimated from the HCIQ. Statistical analysis was performed using SPSS. Regression analysis (Kirkwood 1988) was used to describe the scatter of scores and demonstrate the amount of variation in the scores obtained by the QWB instrument that was explained by the variation in HCIQ estimates. The subjects were divided into two groups on the basis of the order of the administration of the QWB instrument and the HCIQ and regression analysis was performed on each of the subsets as well as on the combined data set. Intra-class correlations (Streiner and Norman 1989) were used 
to exclude the effect of the variation between subjects on the correlation coefficient for the two instruments. The method for describing differences between measures described by Altman (Bland and Altman 1986) was used to summarise the ability of the HCIQ to estimate the scores expected from administration of the QWB instrument. This method uses the mean difference between two sets of readings and the standard deviation of this mean to describe the similarities between two measures. Confidence intervals about the correlation coefficient estimates were calculated using the method described by Streiner and Norman (ie: $\left.N=(z / C I)^{2}+3\right)$

The reliability study results were analysed using the same techniques as those described for the validity study. The practicability of the HCIQ for use in large scale surveys was measured in terms of length of completion time and percentage of complete questionnaires amongst those returned.

\subsection{Results}

\section{The criterion validity study}

There were 211 injured adult subjects registered in the ACTISPP data base during the period of the validity study. Interviews were conducted with 98 of these. There were no significant differences between the sample of people who were interviewed and those who were not for age, sex, nature of injury, type of injury, injury severity, context of injury event and admission status (Table 7.1). All the patients in the study group had injuries that would be scored by the Abbreviated Injury Scale as minor injuries (AIS 1 or 2).

The median completion time of the HCIQ was 15 minutes, with a minimum of 10 minutes and a maximum of 45 minutes. All those who attempted the questionnaire completed the HCIQ satisfactorily without assistance. The QWB scale scores in the sample were normally distributed about a mean of 0.603 with a standard deviation of 
0.127. The estimated QWB scores obtained from the HCIQ were normally distributed about a mean of 0.563 with a standard deviation of 0.137 .

Table 7.1 Distribution of age, sex, nature of injury, body-part injured, Abbreviated Injury Scale (AIS) severity scores, context of injury, and admission status for the criterion-validity-study responders and non-responders.

\begin{tabular}{ccccc}
\hline $\begin{array}{c}\text { Non-responders } \\
(n=113)\end{array}$ & $(\%)$ & $\begin{array}{c}\text { Responders } \\
(n=98)\end{array}$ & $(\%)$ \\
\hline
\end{tabular}

Age (in years)

$\begin{array}{lrrr}16-24 & 53 & (47) & 39 \\ 25-44 & 42 & (37) & 42 \\ 45+ & 18 & (16) & 16 \\ \text { Missing data } & 0 & (0) & 1\end{array}$

(40)

(43)

$\operatorname{Sex}$

$\begin{array}{lrrr}\text { Male } & 69 & (61) & 56 \\ \text { Female } & 44 & (39) & 41 \\ \text { Missing data } & 0 & (0) & 1\end{array}$

Nature of injury

Lacerations
Sprains
Breaks
Other
Missing data

$\begin{array}{rrr}35 & (31) & 25 \\ 58 & (51) & 56 \\ 18 & (16) & 13 \\ 2 & (2) & 3 \\ 0 & (0) & 1\end{array}$

Body part injured

Head/face
Upper limb
Lower limb
Other
Missing data

14
37
55
5
2

\section{AIS scores}

\begin{tabular}{llrrrr} 
& AlS -1 & 85 & $(75)$ & 77 & $(79)$ \\
& AlS -2 & 18 & $(16)$ & 14 & $(14)$ \\
Context & Missing data & 10 & $(9)$ & 7 & $(7)$ \\
& & & & & \\
& Transport & 10 & $(8)$ & 3 & $(3)$ \\
& Occupational & 17 & $(15)$ & 18 & $(18)$ \\
& Sports & 30 & $(27)$ & 22 & $(22)$ \\
& Leisure & 18 & $(16)$ & 17 & $(17)$ \\
& Residential & 35 & $(31)$ & 32 & $(33)$ \\
& Other & 3 & $(3)$ & 6 & $(6)$ \\
\multirow{5}{*}{ Admission } & Missing data & 0 & $(0)$ & 0 & $(0)$ \\
& Yes & 7 & $(6)$ & 3 & $(3)$ \\
& No & 106 & $(94)$ & 94 & $(96)$ \\
& Missing data & 0 & $(0)$ & 1 & $(1)$ \\
\hline $\mathrm{X}^{2} \mathrm{p}>0.05$ for all variables, percents may not add to 100 because of rounding &
\end{tabular}


The distribution of the QWB scores estimated from the HCIQ for all injuries which occurred on more than one occasion in the sample is presented in Table 7.2. The scores represent the transient health state experienced by the subjects at the time of their injury's occurrence. Injuries associated with low QWB scores were therefore more serious than injuries associated with high QWB scores. For example, the immediate health effect of a tibial fracture on the three subjects in the survey who sustained this injury was to deprive them of about $57 \%$ of their optimal quality of life. The immediate health impact of a broken toe on the two subjects who had sustained this injury was about $44 \%$ of their quality of life. The listed QWB scores do not provide information regarding possible ongoing health deficits.

Table 7.2 The mean Health Consequences of Injury Questionnaire ( $\mathrm{HClQ}$ ) estimated score and standard deviations (SD) and number of occurrences $(n)$ by injury type for those injuries occurring on more than one occasion in the sample.

\begin{tabular}{lccc}
\hline Injury type & Mean HCIQ & SD & $\mathrm{n}$ \\
\hline Corneal abrasion & 0.732 & 0.153 & 6 \\
Skin contusion (abdomen) & 0.712 & 0.210 & 2 \\
Skin laceration (face) & 0.688 & 0.096 & 2 \\
Skin contusion (upper limb) & 0.653 & 0.169 & 5 \\
Fractured finger & 0.628 & 0.079 & 3 \\
Skin laceration (upper limb) & 0.627 & 0.175 & 8 \\
Fractured toe & 0.558 & 0.017 & 2 \\
Concussion & 0.517 & 0.151 & 2 \\
Sprained ankle & 0.515 & 0.055 & 15 \\
Skin laceration (lower limb) & 0.512 & 0.050 & 4 \\
Skin contusion (lower limb) & 0.511 & 0.045 & 5 \\
Sprained knee & 0.504 & 0.042 & 7 \\
Sprained wrist & 0.492 & 0.083 & 3 \\
Fractured tibia & 0.430 & 0.019 & 3 \\
Sprained foot & 0.425 & 0.065 & 3 \\
\hline
\end{tabular}


A comparison between the health-index values obtained from the two instruments is illustrated in Figure 7.1. The regression line through these points has a slope of 0.71 $(\mathrm{p}<0.001)$ and an intercept of 0.203 (Table 7.3). There was little effect of order of administration on this regression. The intra-class correlation coefficient between the QWB scale scores and the HCIQ estimated score was 0.86 (95\% CI 0.66-1.00). The mean difference between the scores for each subject was 0.040 with a standard deviation of 0.091 . Thus 95 percent of the HCIQ estimated scores were within 0.182 of the QWB scale scores.

Figure 7.1 Scattergram of Health Consequences of Injury Questionnaire ( $\mathrm{HClQ}$ ) estimates and Quality of Well-Being (QWB) score.

\section{QWB score}

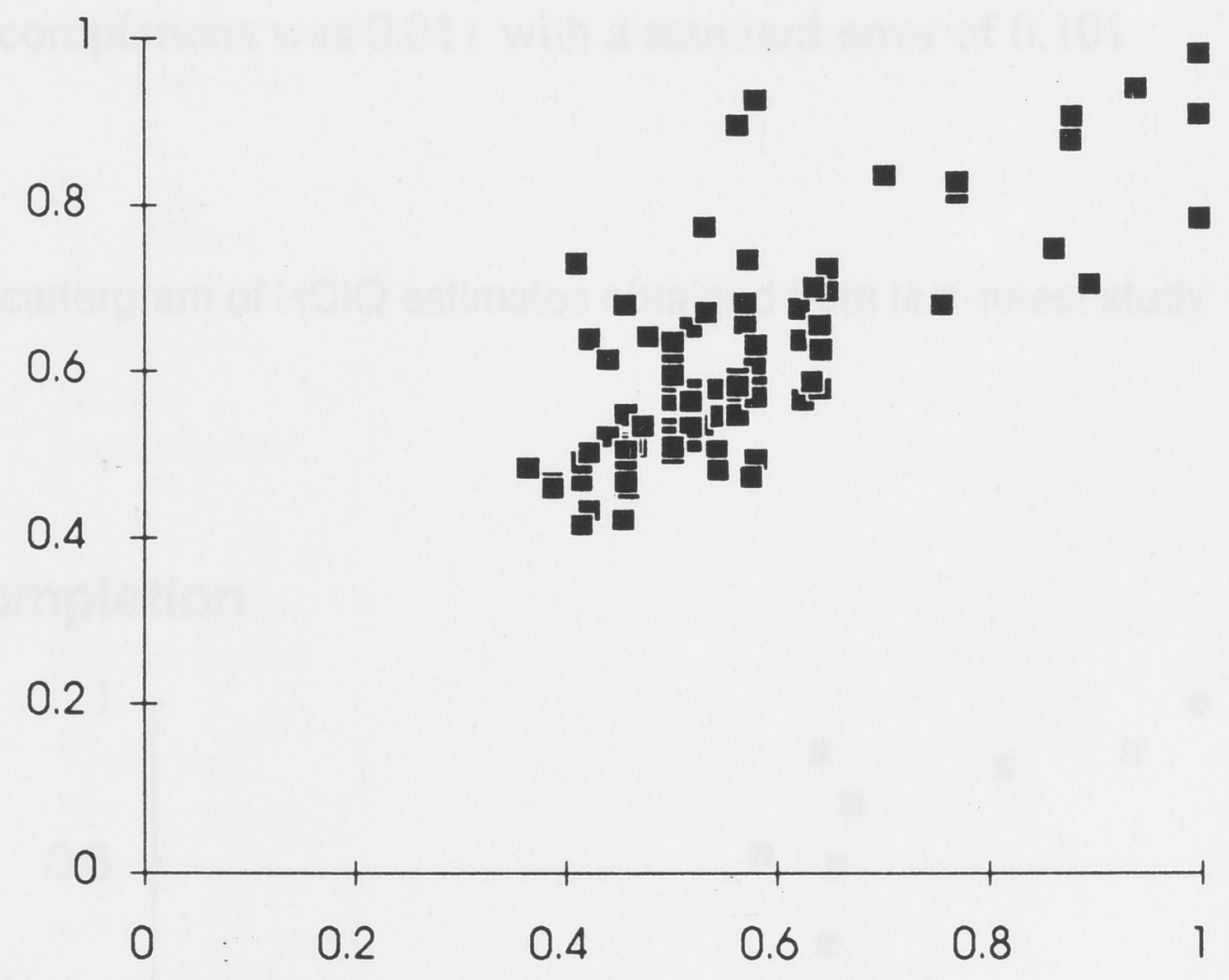

$\mathrm{HCIQ}$ estimate

Table 7.3 Regression of QWB score on HCIQ estimates (QWB score dependant)

\begin{tabular}{lcccc}
\hline & beta & $p$ & $R$ & constant \\
\hline HCIQ administered first $(\mathrm{N}=46)$ & 0.67 & $<0.001$ & 0.83 & 0.205 \\
QWB instrument first $(\mathrm{N}=51)$ & 0.82 & $<0.001$ & 0.73 & 0.156 \\
For total sample $(\mathrm{N}=98)^{\star}$ & 0.71 & $<0.001$ & 0.77 & 0.203 \\
\hline
\end{tabular}

*Order of administration was missing for one case 


\section{Reliability study}

There were 129 injured adult subjects registered in the ACTISPP data base during the period of the reliability study. Fifty-three of the subjects satisfactorily completed two mail-administered HCIQs. There were no significant differences between the sample of people who completed the test-retest and those who did not for age, sex, nature of injury, type of injury, injury severity, context of injury event and admission status (Table 7.4). All the patients in the study group had minor injuries (AIS 1 or 2).

The correlation between the results obtained from the test-retest reliability study are illustrated in Figure 7.2. The regression line has a slope of $0.78(\mathrm{p}<0.001)$ and an intercept of 0.123 . The intra-class correlation coefficient of reliability for the HCIQ is 0.90 (95\% CI $0.62-1.00)$. The mean difference between the scores obtained on the consecutive completions was 0.011 with a standard error of 0.101 .

Figure 7.2 Scattergram of $\mathrm{HCIQ}$ estimates obtained from test-retest study

\section{2nd completion}

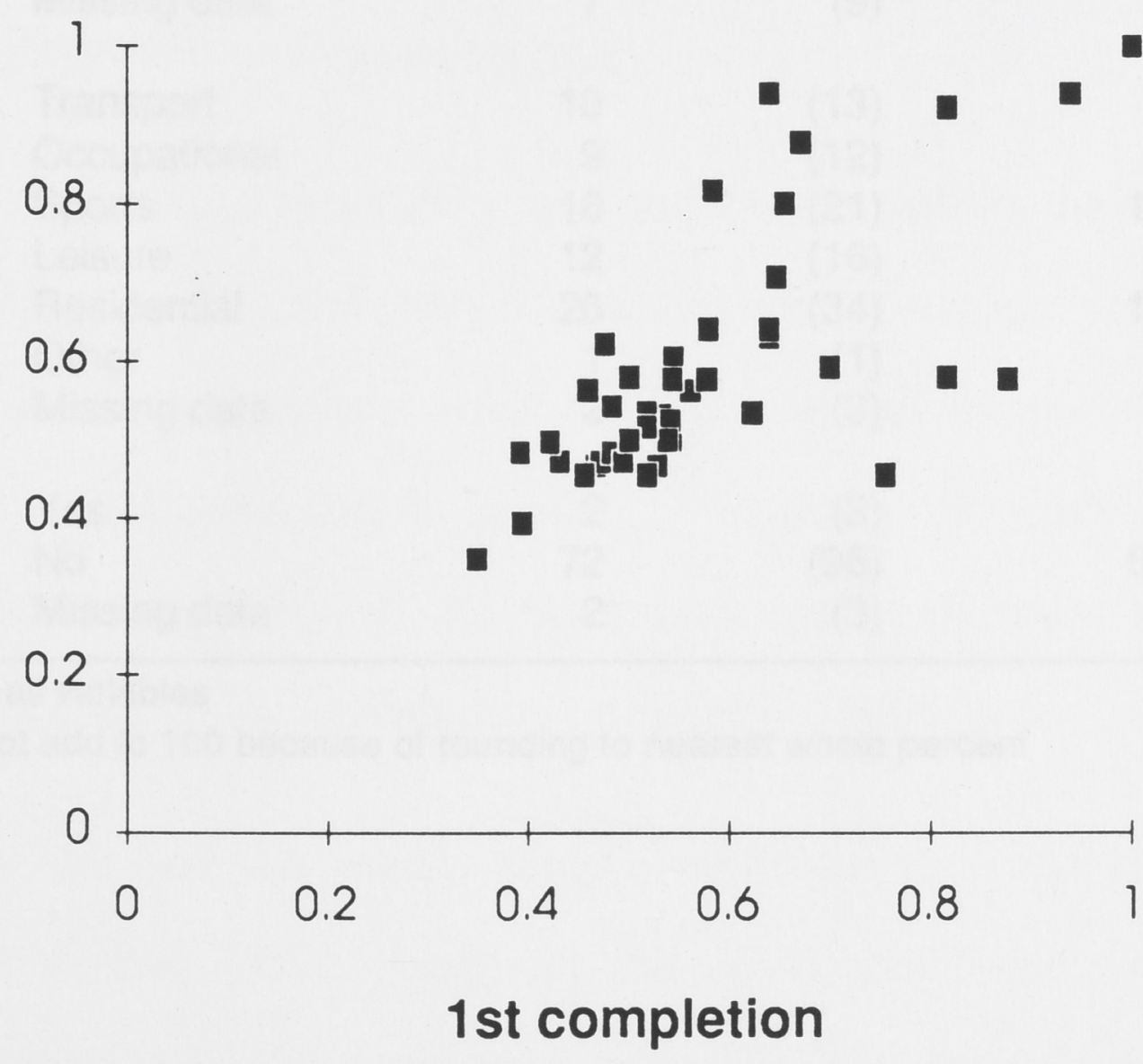


Table 7.4 Distribution of age, sex, nature of injury, body-part injured, Abbreviated Injury Scale (AIS) severity scores, context of injury, and admission status for the reliability-study responders and non-responders.

\begin{tabular}{|c|c|c|c|c|c|}
\hline & & $\begin{array}{c}\text { Non-responders } \\
\qquad(n=76)\end{array}$ & $(\%)$ & $\begin{array}{c}\text { Responders } \\
(n=53)\end{array}$ & $(\%)$ \\
\hline \multicolumn{6}{|c|}{ Age (in years) } \\
\hline & $16-24$ & 36 & $(47)$ & 17 & (32) \\
\hline & $25-44$ & 28 & (37) & 27 & (51) \\
\hline & $45+$ & 10 & (13) & 9 & (17) \\
\hline & Missing data & 2 & (3) & 0 & (0) \\
\hline \multicolumn{6}{|l|}{$\operatorname{sex}$} \\
\hline & Male & 50 & (66) & 30 & $(57)$ \\
\hline & Female & 24 & (32) & 23 & (44) \\
\hline & Missing data & 2 & (3) & 0 & $(0)$ \\
\hline \multicolumn{6}{|c|}{ Nature of injury } \\
\hline & Lacerations & 15 & (20) & 9 & (17) \\
\hline & Sprains & 44 & (58) & 34 & (64) \\
\hline & Breaks & 12 & (16) & 9 & (17) \\
\hline & Other & 5 & (7) & 1 & (2) \\
\hline & Missing data & 0 & $(0)$ & 0 & $(0)$ \\
\hline \multicolumn{6}{|c|}{ Body part injured } \\
\hline & Head/face & 7 & (9) & 7 & (13) \\
\hline & Upper limb & 36 & (48) & 14 & (26) \\
\hline & Lower limb & 27 & (36) & 29 & (55) \\
\hline & Other & 3 & (4) & 3 & $(6)$ \\
\hline \multicolumn{4}{|l|}{ AIS scores } & 0 & $(0)$ \\
\hline & AIS - 1 & 59 & $(78)$ & 44 & (83) \\
\hline & AIS - 2 & 10 & (13) & 6 & (11) \\
\hline & Missing data & 7 & (9) & 3 & (6) \\
\hline \multicolumn{6}{|l|}{ Context } \\
\hline & Transport & 10 & (13) & 2 & (4) \\
\hline & Occupational & 9 & (12) & 8 & (15) \\
\hline & Sports & 16 & (21) & 17 & (32) \\
\hline & Leisure & 12 & (16) & 7 & (13) \\
\hline & Residential & 26 & (34) & 18 & (34) \\
\hline & Other & 1 & (1) & 1 & (2) \\
\hline & Missing data & 2 & (3) & 0 & (0) \\
\hline \multicolumn{6}{|l|}{ Admission } \\
\hline & Yes & 2 & (3) & 2 & $(4)$ \\
\hline & No & 72 & (95) & 51 & (96) \\
\hline & Missing data & 2 & (3) & 0 & (0) \\
\hline
\end{tabular}

$X^{2} p>0.05$ for all variables

Percents may not add to 100 because of rounding to nearest whole percent 


\subsection{Discussion}

The current lack of information on the consequences of non-life-threatening injury is well recognised (National Committee for Injury Prevention and Control 1989). Research is needed to describe the magnitude of the problem at a population level and to identify the determinants of injury-related morbidity. Population-based data is vital to the development and evaluation of control strategies. Such research would be facilitated by the development of a valid and reliable questionnaire suitable for use in large scale epidemiological surveys of injury outcomes.

The QWB score is an ideal unit for measuring the outcomes of injury. The capabilities of the QWB scale in this context have recently been demonstrated in a study of the predictors of functional recovery in a small sample of major trauma patients (Holbrook et al, 1994). Using empirically-derived QWB weights, the health states arising from a range of injury types were scored on a single scale. The subjects had a mean QWB score at discharge of 0.457 (SD 0.048) and a mean score of 0.613 (SD 0.118) three months later. Conceptually, this process is similar to that used to construct the AIS severity scale where injuries are ranked by a panel of experts on a category scale using implicit value weights based on perceived severity. The QWB scale is not suitable, however, for large scale population surveys.

The HCIQ was developed as a survey instrument for measuring the outcomes of injury. The high completion rates and short completion times that were demonstrated for the HCIQ suggest it is a simple instrument and the results of the postal test-retest study, suggest that the instrument is reliable. Indeed, the study results probably underestimate the reliability of the instrument, as recall error would have been responsible for some of the variation between test and retest scores.

The results of the criterion validity study demonstrate the capacity of the HCIQ to predict QWB scores. The strength of the correlation found between the HCIQ estimates and the QWB scores compares favourably with those found in quality-of-life literature, where figures greater than 0.50 are considered to suggest good correlation 
(Lehman et al, 1993). In view of the proven validity of the QWB scale as a health outcome measure which is stable over time but sensitive to changes in health state, this result suggests that repeated administration of the HCIQ over a period of time may be a valid method of measuring injury outcomes. Further studies are needed to confirm this possibility.

Further studies are also needed to address several other questions raised by this preliminary report. The criterion comparison study has provided evidence supporting the validity of the HCIQ. However, the sample size was not sufficiently large to demonstrate the face validity of the HCIQ-estimated health scores. Comparisons between outcomes of the different injury types (Table 7.2) cannot be compared with clinical expectations because there were too few of each injury type in the sample. Testing the HCIQ on a larger sample would be beneficial.

A second issue that must be considered when interpreting the results in this chapter is the low response rates in both the reliability and validity studies. In the reliability study, the response rate was about $65 \%$ for each mailing of the HCIQ resulting in $41 \%$ of the original sample completing both questionnaires. The low final response rate left a smaller than expected sample size and therefore resulted in wide confidence intervals around the reliability coefficient. Nevertheless, even at the extremes of these limits the reliability coefficient was satisfactory. It is unlikely that the low response rate introduced a differential bias in the results as analysis of the responders and the nonresponders (Table 7.4) showed no differences between the two groups.

The response rate in the validity study was $46 \%$. This low response rate was a reflection of the stringent requirements of the QWB interview schedule. For the QWB scale to measure the outcome of a specific injury it must be administered between four and six days after the injury's occurrence. If the research assistants and subjects were unable to arrange an interview within this two-day period then the subject was recorded as a non-responder. It is unlikely that the low response rate introduced a 
differential bias in the results as analysis of the responders and the non-responders (Table 7.1) showed no differences between the two groups.

Generalisation of the results of the criterion study must be made with some caution. In the regression equations comparing the QWB scale scores with the HCIQ estimates, it appears that when the HCIQ predicts a score of zero (representing death) the QWB scale would record a score of about 0.200 . This intercept bias is, in part, a consequence of extrapolating values beyond the range of the study sample. The HCIQ was tested in a sample of injured people over the age of 16 who had a literacy in English and who had injuries that were not severe enough keep them in hospital for more than four days. The criterion validity of the HCIQ has not been established for use in other circumstances.

A second limitation of the generalisability of the HCIQ criterion validity is reflected in the slope bias of the regression analysis and is illustrated in Figure 7.1. Those subjects with the greatest difference between the two scores are represented on the scattergram by a cluster of points with high QWB scale scores and a low HCIQ estimates. This group includes a concussion and several minor, soft tissue injuries and probably represents that group of rapidly healing injuries where the averaging process of the QWB scale results in a higher QWB score than the 'worst state' scoring method of the HCIQ.

A further explanation for some of the discrepancy between the HCIQ estimates and the QWB scores lies in the fact that the QWB is a general health status measure and the HCIQ specifically identifies health loss from injury. Those people in the study who had health defects caused by other illnesses as well as injury would be assigned a lower score by the QWB scale than by the HCIQ. However, as the large majority of subjects in the sample were young and fit this is not believed to have appreciably distorted the results.

The HCIQ is a simple questionnaire designed for self-completion that identifies a subject's injury-related health profile. The preliminary studies described in this chapter 
show the HCIQ to be practical and reliable. When scored with QWB weights the HCIQ can be validly used to predict QWB health index values. 


\section{Chapter Eight}

\section{Health outcomes of injury in the Australian Capital Territory}

\subsection{Introduction}

Injuries are the leading cause of death in Australia for males aged under 45 years and females aged under 30 years. Injury is also the leading cause of hospitalisation in people less than 45 years of age (AIHW 1992). Evidence discussed in the literature review suggests that these mortality and hospitalisation figures represent only the tip of the iceberg and that the outcomes of non-life-threatening injuries make a considerable impact on the health of individuals in a community. The extent of the community burden arising from minor injury has not previously been quantified.

Chapter Five described the incidence of injury occurring in an Australian population. For the Australia Capital Territory (ACT) during 1992 the all-age all-injury ratio of deaths to admissions to emergency department presentations to GP attendances for new non-intentional injuries was found to be $1: 28: 596: 592$. If the long-term health consequences of minor injuries are substantial, then by weight of numbers, these injuries could be expected to account for a considerable portion of the total injury burden in the community.

The aim of this chapter is to estimate the incidence-based injury morbidity in the ACT adult population during 1992. 


\subsection{Methods}

\subsubsection{Study design}

A prospective cohort study was conducted using a sample of injured adults to identify the health outcomes arising from the range of injuries in the study population. The total health loss due to injury experienced by these subjects over the period of injury recovery was calculated. The subjects in the follow-up sample were weighted, using the estimates derived from data presented in Chapter Five, to produce incidence-based estimates for the total morbidity in the ACT adult population arising from new injuries treated in the emergency departments in 1992. The total injury-related morbidity was then stratified by relevant variables to identify the injury types and demographic groups responsible for the major components of this burden.

\subsubsection{Participants and process}

\section{Incidence samples}

Information about the expected population incidence and distribution of injury was drawn from emergency departments and general practitioners as described in detail in Chapter Five. The study population included ACT residents, 16 years or older, who received medical care for a new injury in either hospital emergency departments or general practices in the ACT between 12 March 92 and 11 March 1993.

\section{Follow-up sample}

Information about the morbidity arising from injury was obtained from subjects in the follow-up sample. The subjects for this sample were identified through the Australian Capital Territory Injury Surveillance and Prevention Project (ACTISPP) described in Chapter Six. Those subjects included in the follow-up sample for this study were all people 16 years or older who registered in the ACTISPP survey from 12 March 1992 
to 11 May 1992 and from 12 June 1992 to 11 September 1992, who had a condition classifiable by ICD-9 codes $800-995$, and who agreed to participate in the study. Data collected included age, sex, postcode of residence, nature of injury, body part injured and discharge status. For the subset of subjects registered in the study between 12 March and 11 April 1992 information regarding pre-existing medical conditions was also obtained.

The outcome variables were obtained by means of repeated administrations of the Health Consequences of Injury Questionnaire (HCIQ) described in Chapter Seven. The outcome ranges from zero to one where one represents full health and zero represents death. One minus this index represents the health loss due to the given injury.

There are two forms of the HCIQ which were scored using the same procedures. The first was administered one week after the injury event and instructs subjects to recall limitations experienced in the week since their injury. The second version was used for the follow-up administrations of the questionnaire and asked for limitations experienced during the week preceding the repeat completion. Time intervals for the mailings of this questionnaire were one week, six weeks, three months and six months after injury occurrence. Two reminder letters were sent to non-responders. Follow-up ceased when the subject reported injury recovery or if the subject failed to respond to the second reminder. Those subjects not yet recovered at six months were censored. Patient identifying particulars were stored in a discrete software system responsible for managing the mailing process. The subjects' health details were stored on separate files using random identification numbers to protect patient privacy. The follow-up process and coding was managed by a trained research assistant.

The follow-up sample was selected from the emergency departments of the ACT while the study population included all those injuries treated by both GPs and emergency departments in the ACT. For the purposes of the study it was assumed that the outcomes of a given injury would depend upon the nature of injury and this outcome 
would be the same whether the injury was treated in the emergency department or general practice.

\subsubsection{Analysis}

The method of calculating the total health loss due to injury over time until recovery is illustrated in Figure 8.1. Time ' 0 ' is the point at which the injury is sustained and time ' $\mathrm{T}$ ' the point at which the injury recovers. The vertical axis records the health loss at any given point in time. Repeated administrations of the HCIQ over the recovery period enabled each subject's health loss to be plotted over time. The area enclosed by this curve represents the accumulated health loss due to injury and was deemed to be the morbidity arising from the injury.

This quantity can be expressed in the formula

$$
\int_{0}^{T} H C I(t) d t
$$

With time being measured in years, the units for the area enclosed by the curve are quality adjusted life years.

Figure 8.1 Graph of health loss against time for a hypothetical injury which occurred at time ' $O$ ' and recovered at time ' $T$ ' to illustrate definition of injury morbidity (ie the accumulated health loss due to injury over time until recovery).

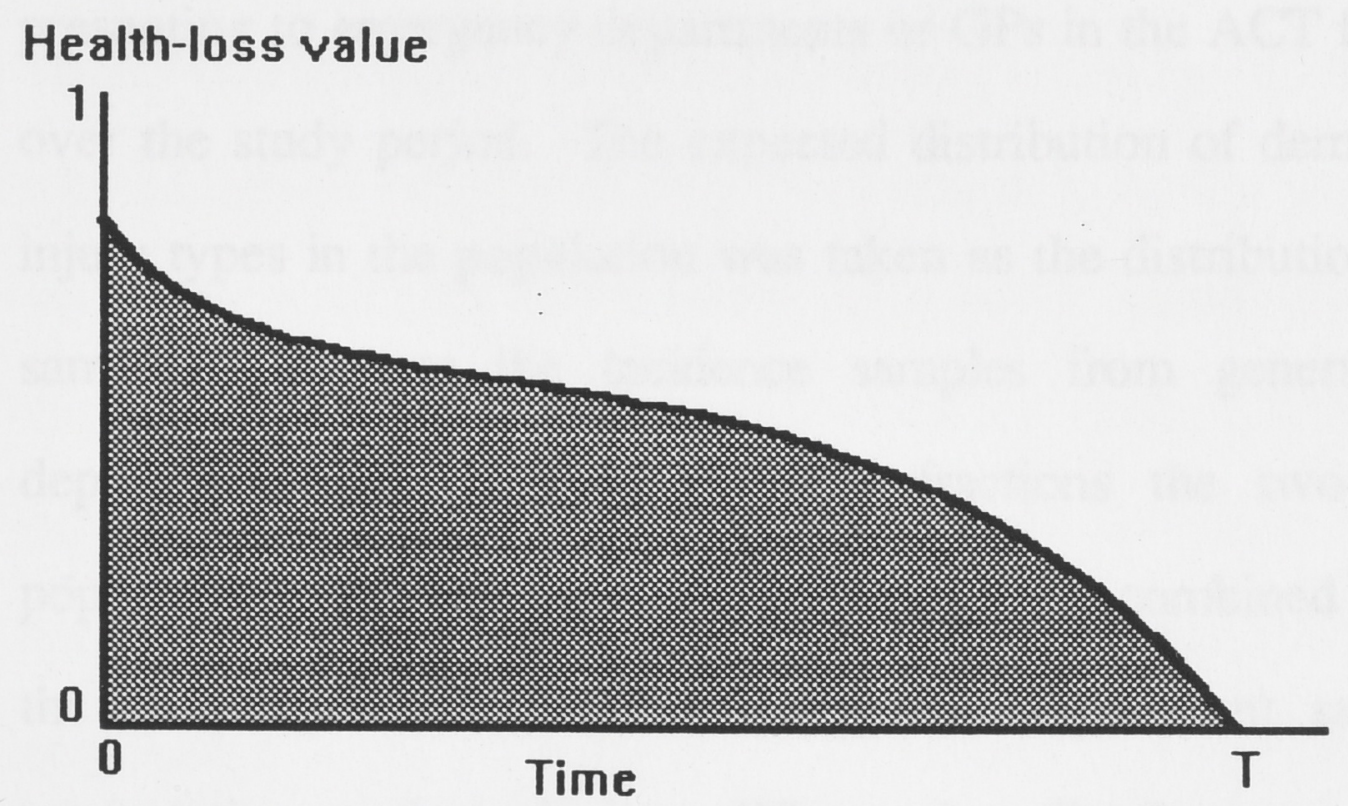

Ideally, all subjects in the sample would have been followed until their injury recovered. This, however, was clearly impractical as some of the injuries were expected to result in permanent disability. It was anticipated that most injuries would recover within six months of the injury event and that a large number of those not 
recovered by this time would result in some degree of permanent disability. It was anticipated that the survival curve drawn for the sample, where 'injury recovery' was the failure point, would describe an exponential decay function that would reach a minimum-value asymptote at six months. On this reasoning the follow-up period of the study was set at six months and the contribution of the unrecovered injuries towards a lifetime-morbidity estimate was justified on the basis of an extrapolation of the six month survival curve. Those subjects in the follow-up sample who had not recovered at six months had a life-time-morbidity value calculated on the assumption that the injury-related compromise to their health status would remain constant throughout their remaining years.

Quality-adjusted life-years lost were calculated for the unrecovered group by multiplying their health loss value at six months by the difference between their age and their life expectancy. Life expectancy for each patient was obtained from the 1992 life tables (ABS 1993). Thus, the morbidity values calculated for the first six months after injury were based on observed data, while the life-time morbidity estimates were predictions of probable outcomes.

The incidence sample was used to estimate the total number of adult ACT residents presenting to emergency departments or GPs in the ACT for treatment of a new injury over the study period. The expected distribution of demographic characteristics and injury types in the population was taken as the distribution in the combined incidence sample. Because the incidence samples from general practice and emergency departments had different sampling fractions the two samples were inflated to population estimates before combination. The combined sample was then divided by the sampling fraction of the emergency department sample (ie renormalised) for presentation and analysis. Where the distribution of these injury types and demographic variables in the follow-up sample was found to differ from the expected distribution, weights were applied to the follow-up data to make it more closely reflect expected distribution. The weighting process also inflated numbers in the sample to the estimated incidence of injury in the community. Thus the morbidity calculated for 
the weighted follow-up sample represents the estimated total incidence-based community morbidity arising from injury in the ACT during 1992.

Deaths of those ACT residents aged 16 years or older that occurred during 1992 and were due to an ICD-9 external cause 800-995 were also included in the analysis for comparison purposes (ABS 1993). The mean age at death by sex was provided by the ABS for this group. The total number of potential years of lost life in this group was taken as the difference between the group mean age at death for males and females and the median Australian population age at death in 1992, multiplied by the number of people of each sex in the group. The median age at death for Australian males in 1992 was 72.6 years old and for females it was 79.3 years.

\subsection{Results}

There were an estimated $32,400(95 \% \mathrm{CI}=31,700$ - 33,100) first-presentation injuries in people 16 years and over presented to general practitioners or emergency departments in the ACT during the study period. Renormalising this resulted in a combined incidence sample of 2,312 subjects. The characteristics of this expected population distribution is listed in Table 8.1.

There were 2,823 adult ACT residents registered by ACTISPP during the study period who had given their consent for involvement in further studies and those subjects were invited to participate in the follow-up process. Of those, 1396 chose to participate in the follow-up study. The responder bias for those ACTISPP registrants who chose to participate in the follow-up study was modelled using logistic regression using the variables age, sex, nature of injury, body-part injured and whether hospitalised. The respondents were more likely to be younger (OR 1.2, 95\% CI 1.1-1.3), and female (OR 1.5, 95\% CI 1.3-1.7) than the non-respondents. No differences were found for the other variables. 
Table 8.1 Distribution of age, sex, nature of injury, body part injured and whether hospitalised in the incidence and follow-up samples.

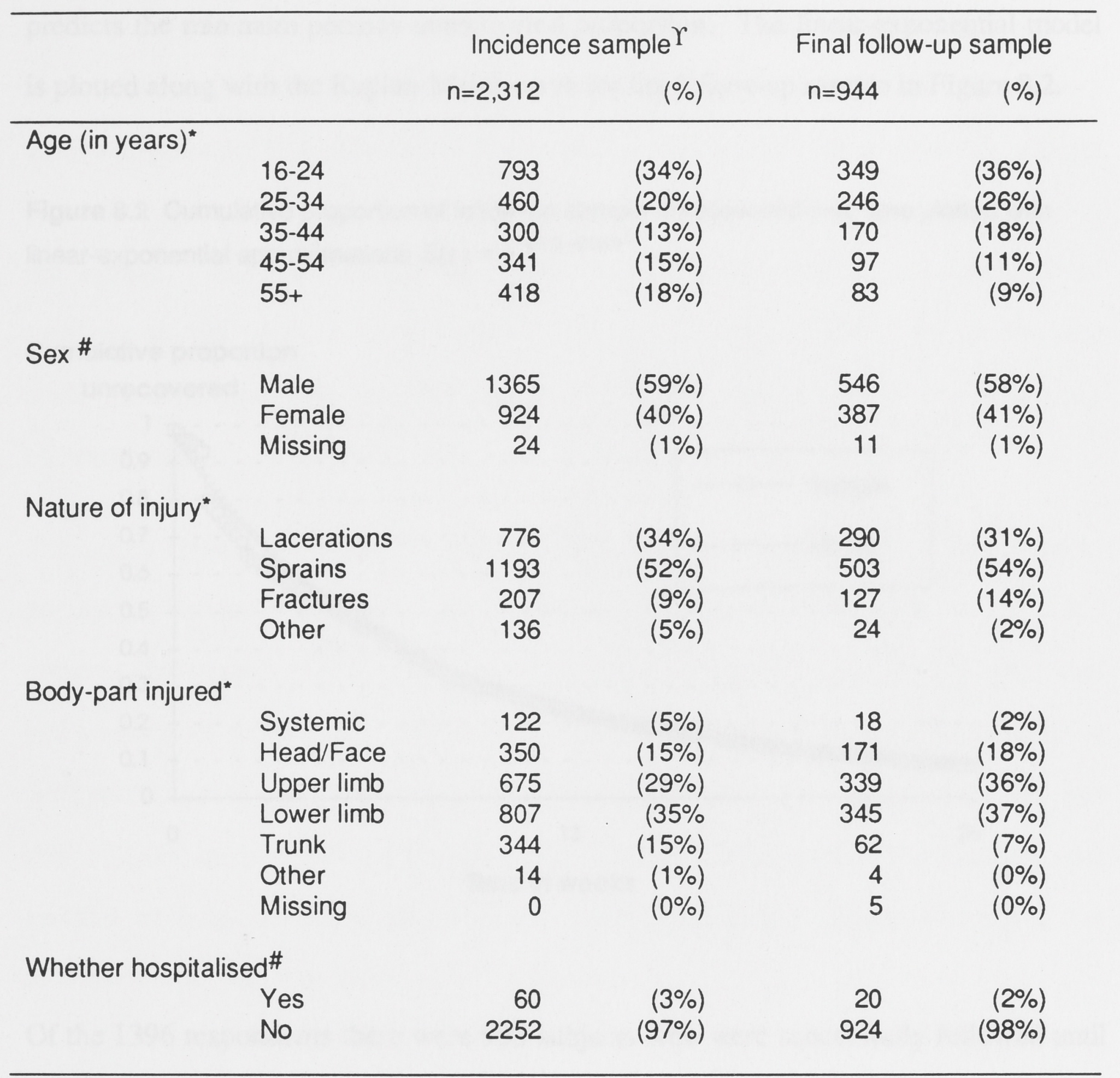

$\Upsilon$ renormalised combined weighted emergency department and general practice samples

* $\mathrm{X}^{2} \mathrm{p}$ value $<0.001,{ }^{\#} \mathrm{X}^{2} \mathrm{p}$ value $>0.05$, percents may not add to 100 because of rounding

The Kaplan-Meier survival curve of the 1396 respondents was plotted, with injury recovery being the registered failure point. The resulting curve was closely approximated by an exponential model $S(t)=0.087+e^{-0.205 t}$ which reaches a minimum-value asymptote of 0.087 as time approaches infinity but which, for all practical purposes, reaches this value at the time of six months. The survival curve was also closely approximated by a linear exponential model $S(t)=e^{-0.17 t-0.003 t^{2}}$. This model also has a minimum value of 0.087 at the time of six months however because of the quadratic component of the equation it describes an increase in the $S(t)$ value once the minimum is passed. As this mathematical increase is incompatible with real world 
circumstances the model was considered to be applicable only to the point where it predicts the minimum persons-unrecovered proportion. The linear-exponential model is plotted along with the Kaplan-Meier curve for the follow-up sample in Figure 8.2.

Figure 8.2 Cumulative proportion of follow-up sample unrecovered over time plotted with linear-exponential approximations $S(t)=e^{-0.17 t-0.003 t^{2}}$

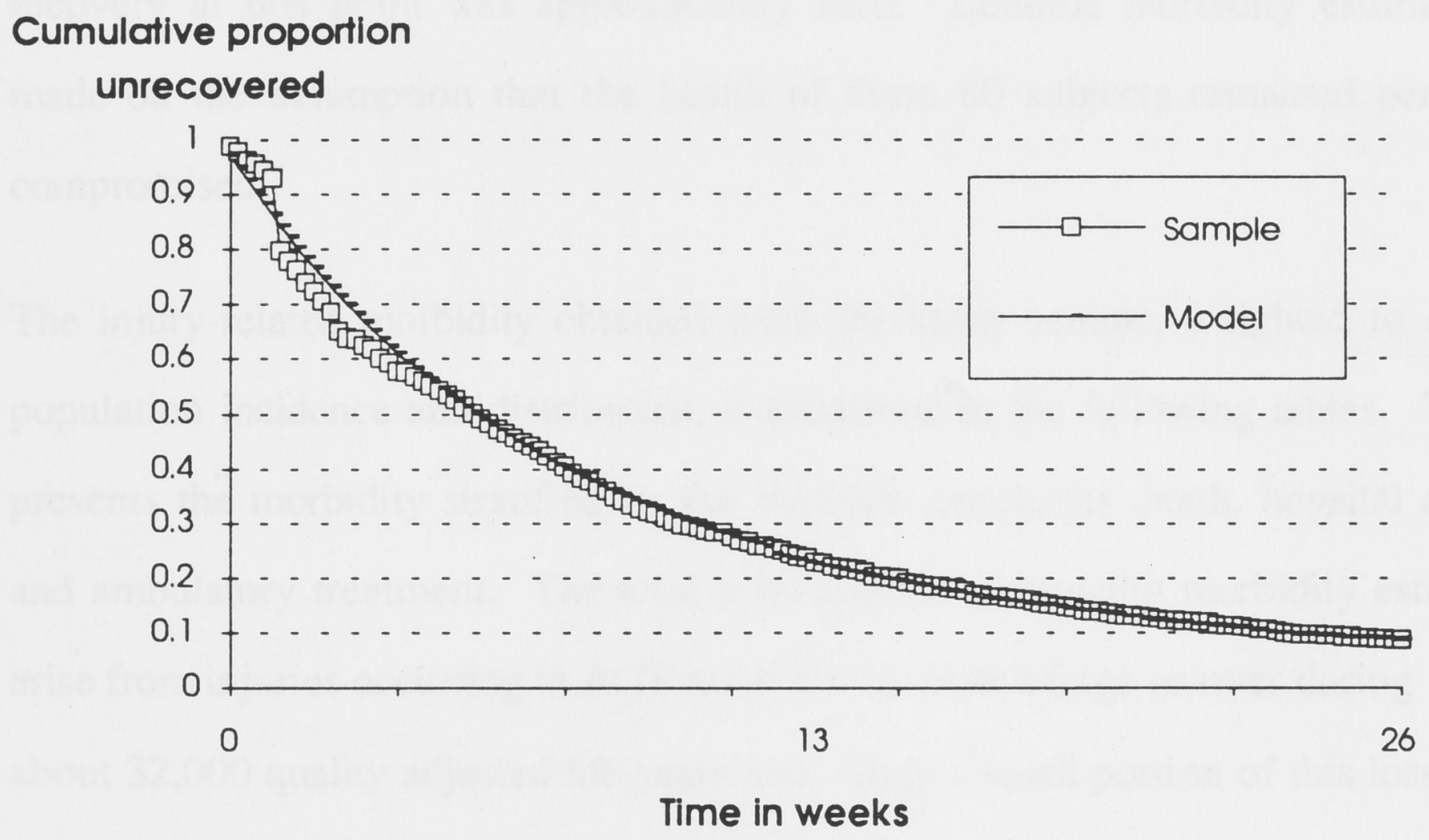

Of the 1396 respondents there were 953 subjects who were successfully followed until their injury had recovered or censorship at six months. There were no significant differences between the group that completed follow-up and those that dropped out for any of the variables - age, sex nature of injury, body part injured and whether hospitalised. The characteristics of the final 953 subjects who completed follow-up are presented in Table 8.1 and the differences between this group and the incidence sample are compared. The differences are statistically different for age, nature of injury and body part injured. The magnitude of the differences however, are small. Weights were calculated for the morbidity calculation described below so that the age and body part injured distribution in the final follow-up sample conformed to the distribution in the incidence sample. Weights also included an inflation factor that ensured the weighted sample size equalled the estimated injury incidence in the population. 
Because the morbidity variable was an area under a health loss curve plotted over time until recovery or censorship at six months, only those subjects who completed the follow-up process could be used in the morbidity calculations. Of the 953 subjects who were successfully followed until recovery at six months, there were 80 subjects who remained unrecovered at the end of this follow up period, representing $8.5 \%$ of the sample. As described by the models discussed above, the modelled rate of recovery at this point was approximately zero. Lifetime morbidity estimates were made on the assumption that the health of these 80 subjects remained permanently compromised.

The injury-related morbidity obtained from the study sample, weighted to estimated population incidence and distribution, is presented in the following tables. Table 8.2 presents the morbidity stratified by the outcome categories death, hospital admission and ambulatory treatment. The total accumulated community morbidity estimated to arise from injuries occurring in ACT residents 16 years of age or over during 1992 was about 32,000 quality adjusted life years lost. Only a small portion of this loss was due to fatal or hospitalised injuries with the ambulatory injured group accounting for morbidity that was an order of magnitude larger.

Table 8.2 Estimated total quality adjusted life-years (QALYS) lost in the Australian Capital Territory due to injury in adults during 1992 by outcome category (deaths, hospital admissions, and ambulatory treatment groups).

\begin{tabular}{lcc}
\hline Injury category & $\begin{array}{c}\text { QALYs lost over } \\
\text { first six months }\end{array}$ & $\begin{array}{c}\text { QALYs lost over } \\
\text { life time }\end{array}$ \\
\hline Deaths & 17 & 933 \\
Hospital admissions & 100 & 3,070 \\
Ambulatory treatment & 1,150 & 28,429 \\
Total & 1,267 & 32,432 \\
\hline
\end{tabular}


Table 8.3 presents the injury-related outcomes by nature of injury. About half the total morbidity arising from injury in the ACT was due to sprains in the 16-34 age group; then, in order of diminishing contribution came breaks, lacerations and other injuries. Although injuries to males in the ACT during 1992 outnumbered injuries to females in a 1.7:1 ratio (Chapter Five), over half the total disability arising from injuries in the community was experienced by women.

Table 8.3 Estimated total quality adjusted life-years (QALYS) lost in the Australian Capital Territory due to non-fatal injury in adults during 1992 by nature of injury, stratified by age and sex.

\begin{tabular}{|c|c|c|c|c|c|c|}
\hline \multirow{2}{*}{$\begin{array}{c}\text { Age } \\
\text { (in years) }\end{array}$} & & \multicolumn{2}{|c|}{$\begin{array}{l}\text { QALY's lost over first } \\
\text { six months }\end{array}$} & \multicolumn{3}{|c|}{ QALY's lost over life time } \\
\hline & & Male & Female & Male & Female & Total \\
\hline \multicolumn{7}{|l|}{$16-34$} \\
\hline & laceration & 52 & 26 & 1,299 & 374 & 1,673 \\
\hline & sprain & 219 & 236 & 5,546 & 10,469 & 16,015 \\
\hline & break & 72 & 73 & 2,921 & 1,960 & 4,881 \\
\hline & concussion & 3 & 21 & 3 & 21 & 24 \\
\hline & other & 0 & 3 & 0 & 3 & 3 \\
\hline \multirow[t]{5}{*}{$35-54$} & laceration & 58 & 26 & 2,039 & 467 & 2,506 \\
\hline & sprain & 85 & 117 & 983 & 2,975 & 3,958 \\
\hline & break & 38 & 35 & 788 & 849 & 1,637 \\
\hline & concussion & 6 & 1 & 6 & 1 & 7 \\
\hline & other & 3 & 0 & 3 & 0 & 3 \\
\hline \multirow[t]{6}{*}{$55+$} & laceration & 13 & 47 & 13 & 47 & 60 \\
\hline & sprain & 25 & 18 & 25 & 18 & 43 \\
\hline & break & 17 & 51. & 17 & 672 & 689 \\
\hline & concussion & 0 & 0 & 0 & 0 & 0 \\
\hline & other : & 0 & 0 & 0 & 0 & 0 \\
\hline & & & & 13,643 & 17,856 & 31,499 \\
\hline
\end{tabular}

Note: Total QALY's are 8 short of expected because missing value cases were excluded from table.

Table 8.4 presents the injury-related outcomes by body-part injured. About one third of the total morbidity arising from injury in the ACT was due to lower limb injuries in the 16-34 age group; then, in order of diminishing contribution came upper limb, trunk, face and other injuries. 
Table 8.4 Estimated total quality adjusted life-years (QALYs) lost in the Australian Capital Territory due to non-fatal injury in adults during 1992 by body-part injured, stratified by age and sex.

\begin{tabular}{|c|c|c|c|c|c|c|}
\hline \multirow[t]{2}{*}{$\begin{array}{l}\text { Age (in } \\
\text { years) }\end{array}$} & & \multicolumn{2}{|c|}{$\begin{array}{l}\text { QALY's lost over first } \\
\text { six months }\end{array}$} & \multicolumn{3}{|c|}{ QALY's lost over life time } \\
\hline & & Male & Female & Male & Female & Total \\
\hline \multirow[t]{6}{*}{$16-34$} & systemic & 3 & 23 & 3 & 23 & 26 \\
\hline & face & 22 & 30 & 22 & 861 & 883 \\
\hline & upper limb & 129 & 102 & 3,188 & 4,496 & 7,684 \\
\hline & lower limb & 153 & 149 & 5,387 & 5,741 & 11,128 \\
\hline & trunk & 39 & 52 & 1,174 & 1,705 & 2,879 \\
\hline & other & 0 & 0 & 0 & 0 & 0 \\
\hline \multirow[t]{6}{*}{$35-54$} & systemic & 7 & 1 & 7 & 1 & 8 \\
\hline & face & 51 & 12 & 1,438 & 12 & 1,450 \\
\hline & upper limb & 50 & 64 & 1,547 & 1,849 & 3,396 \\
\hline & lower limb & 66 & 73 & 811 & 1,329 & 2,140 \\
\hline & trunk & 16 & 28 & 16 & 1,101 & 1,117 \\
\hline & other & 0 & 0 & 0 & 0 & 0 \\
\hline \multirow[t]{6}{*}{$55+$} & systemic & 1 & 10 & 1 & 10 & 11 \\
\hline & face & 7 & 19 & 7 & 206 & 213 \\
\hline & upper limb & 19 & 81 & 19 & 515 & 534 \\
\hline & lower limb & 28 & 6 & 29 & 6 & 35 \\
\hline & trunk & 0 & 0 & 0 & 0 & 0 \\
\hline & other & 0 & 0 & 0 & 0 & 0 \\
\hline Total & & & & 13,649 & 17,855 & 31,504 \\
\hline
\end{tabular}

Note: Total QALY's are 3 short of expected because missing value cases were excluded from table.

Table 8.5 presents the morbidity stratified by injury severity. During the first six months after injury those injuries categorised by the AIS as minor injuries account for four times more morbidity in the community than the more severe injuries. This relationship remains for extrapolated lifetime health loss.

Table 8.5 Estimated total quality adjusted life-years (QALYS) lost in the Australian Capital Territory due to non-fatal injury in adults during 1992 by Abbreviated Injury Scale (AIS) severity score.

\begin{tabular}{lcc}
\hline Injury Severity & $\begin{array}{c}\text { QALY's lost over first six } \\
\text { months }\end{array}$ & QALY's lost over life time \\
\hline AIS $>1$ & 242 & 8,238 \\
AIS 1 & 1,008 & 23,269 \\
\hline
\end{tabular}




\subsection{Discussion}

This study has shown that minor injuries are responsible for the greater part of the injury-related health burden in the adult community. Injuries that can be coded as AIS1 account for $80 \%$ of the morbidity arising over the first six months after injury and about $75 \%$ of the estimated lifetime morbidity. The lifetime estimates of morbidity due to injuries not considered serious enough to admit to hospital were of an order of magnitude higher than those that resulted in either death or hospitalisation. Sprains contributed as much to the total morbidity as all other injuries types combined and limb injuries contributed more to the total morbidity than injuries to the more central structures.

The results of this study need to be qualified by the acknowledgment of several of the study's limitations. First, the final sample used to calculate the ACT morbidity constituted only $30 \%$ of those people initially approached. Despite the low response rate there was no statistically significant difference between the follow-up sample and the expected population distribution by sex or whether hospitalised for initial treatment. It is probable that those subjects with major traumas who sustained multiple injuries would have been too unwell at the time of the presentation to participate in the follow-up sample. Thus, with the most severely injured patients not being included in the sample, the morbidity arising from hospitalised patients was probably underestimated. Statistical differences existed between the follow-up sample and the expected population distribution by nature of injury, age and body part injured. In the case of nature of injury, these differences were small (less than $9 \%$ for any category) and would not have affected the results to any great extent. For age and body part injured, where the differences were greater, the follow-up sample was weighted to the expected distribution to overcome the differential response bias introduced. It is possible the follow-up sample differed from the population in other ways not controlled in this study which may have affected the results. Those who replied may, for example, have had an exaggerated health interest and hence the study results may have overestimated the morbidity in the population. 
Second, the population morbidity was based on results of a follow-up sample that was drawn from emergency departments although almost half the injuries in the community receive initial management in general practice. In this study it was assumed that the outcomes of a given injury would depend upon the nature of injury and this outcome would be the same whether the injury was treated in the emergency department or general practice. If this were not the case then differential bias would affect the validity of the results. Possible biases introduced here include the differential effects of management at the two places of treatment. A second possibility is the different severities of presenting injuries at the hospital and general practices.

A third restriction on the strength of the claims that can be made on the basis of the study results, derives from the validity of extrapolating lifetime outcomes from six months of data. The extrapolations were based upon the assumption that injuries not healed at six months result in some health loss that persists throughout the subjects' lives. Objections to this assumption may be raised on the grounds that there are many injuries which are not healed at six months but which do go on to recover to an extent where there are no signs of the injury having occurred. If this were true, the lifetime morbidity calculated in this study may be an overstatement of the real situation. However, the basic relationships between the morbidity resulting from the different categories of injury remains unchanged even if only a quarter of the observed "unrecovered" group had persistent problems. It is not the actual morbidity estimates in this paper which are used to demonstrate the importance of minor injury but the orders of magnitude which separate the outcomes of the different categories of injury. These differences are so great as to be left unaffected by minor changes to the underlying assumptions on which the calculations are based and hence are highly persuasive.

The lifetime estimates were derived from those 80 subjects who had not recovered six months after their injury occurred. When this group is stratified by age, sex, nature of injury or body part injured there are sometimes few subjects in each cell. In some 
cases therefore, selected individuals have an inordinate effect on the final results and hence the results should be interpreted with caution.

The results of this study support the recognition of the public health importance of minor injury. With minor injury probably accounting for the greater part of the total health burden arising from injury, strategies for the prevention and management of minor injury must be included in national programmes for injury control. However, medical outcomes constitute only part of the total public health impact of minor injury. Quantifying the public health impact of a condition must include the measurement of the extent to which finite health resources are consumed. The following chapter considers the economic cost of the health resources involved in the initial management of minor injury. 


\section{Chapter Nine}

\section{Economic cost of minor injuries in the Australian Capital Territory}

\subsection{Introduction}

In Chapter Five it was shown that the vast majority of people with injuries presenting to hospital emergency departments for initial assessment receive definitive treatment in the department and are not admitted to hospital. Chapter Five also demonstrated that as many as half of the medically treated injuries in the community attend general practitioners (GPs) rather than emergency departments for initial treatment. There have been no published Australian studies which have documented the cost of managing acute injury in hospital emergency departments. Nor have there been any studies which have described the economic costs of managing acute injury in general practice.

The most recent estimates of the direct cost of injuries in Australia (AHMAC/NH\&MRC working groups 1994) were derived from total hospital outpatient costs collected by the Australian Institute of Health and Welfare (AIHW). The AIHW estimated that in 1992, the cost of all hospital care throughout Australia was $\$ 9,206$ million. It was estimated that $2.9 \%$ of this cost was for provision of 3.3 million emergency department services. A current price estimate of $\$ 81$ was calculated for each emergency department visit. There are several limitations with using this method to obtain the cost of injury treatments in hospital emergency departments. First, the estimates were average emergency department attendance costs and were not specific for injured patients. Second, the estimates were obtained by averaging the cost of all outpatient department services, including those provided by specialists clinics, which provide vastly different services to those offered in the emergency department. A further disadvantage of these methods of deriving emergency department costs is 
that they are quite dissimilar to any method that could be used to estimate the costs of treating injury in general practice. This dissimilarity in methodology hinders useful comparisons between emergency department and general practice components of the economic burden of injury because of the vastly different unit costs involved that cannot be included in the estimates.

The aim of this chapter is to estimate the incidence-based economic cost of acute treatment of minor injury in the ACT. Two studies are described. The first estimates the cost of treating injury in general practice and the second establishes the cost of treating minor injury in the hospital emergency departments in the ACT.

\subsection{General practice study}

\subsubsection{Background}

The cost of treating injury in general practice is based upon secondary analysis of data from the Australian Morbidity and Treatment Survey 1990-1991 (AMTS) (BridgesWebb et al. 1992). This survey was a stratified, random sample survey of general practitioners (GPs) in Australia conducted from October 1990 to October 1991. Its objective was to provide up-to-date information for medical education and provision of health services and to provide reference data for smaller purpose-specific studies.

In the AMTS, a GP was defined as a medical practitioner working a fee-for-service system who provided more than 1500 items of general practice service per year, and for whom these items represented more than $50 \%$ of their work load. The researchers drew a sample of such GPs from the 1989 Health Insurance Commission files. During two, one-week periods, (staggered so that the total sample covered the twelve month study period), participating GPs recorded details of all their doctor-patient encounters. nursing home and institutional visits were not included in the survey. The details recorded for each encounter included date of encounter, Medicare item number (only one was recorded for each visit), patient age and sex, whether seen before in the 
practice, patient reasons for encounter, problems managed during the encounter, whether each problem was new or old, treatments performed and medications prescribed, investigations ordered, and admissions, referrals and follow-up consultations arranged. If several problems were managed during one encounter, details of up to four of these were recorded. The process of data collection maintained direct links between problems, treatments and prescriptions, but not between problems and item numbers, referrals, tests and investigations (Appendix - 6).

The AMTS was not designed to answer the questions posed in this chapter and consequently techniques needed to be developed to overcome several limitations. For example, where the single Medicare item number recorded in the survey did not directly reflect all the treatments provided, and the treatments were obviously related to a new injury, then additional item numbers were assigned to the treatments for new injuries and their cost estimated. Where the information recorded in the database was not directly linked to the problems managed, as was the case for tests and referrals, indirect links were established using assumptions based upon normal clinical practice.

The reference population for the secondary analysis was considered to be those people who presented to general practice for primary care of a new injury; whether the injury was the sole problem managed at that encounter or the injury was managed in conjunction with one or more other problems. This specification excluded those injuries first treated in hospital emergency departments or by another medical practitioner and referred to general practice for follow up.

Injury was defined as any condition which could be classified by the International Classification of Diseases - 9th revision (ICD-9) from 800 to 995 . However, in the AMTS the problems managed in general practice were classified according to the International Classification of Primary Care (ICPC) (Lamberts and Woods 1987) which does not easily translate into ICD-9 groupings. Furthermore, there is no single heading in the ICPC under which injuries are listed. For the purposes of this study, therefore, injuries in the AMTS database were identified by selecting those ICPC codes for 
conditions which fell within the scope of the ICD-9 definition. The ICPC codes used to identify injuries in the AMTS database included: A;80,81,88, B;76,77, D;79,80, F;75,76,79, H;76-78, L;72-75,77-81,96, N;79-81, R;87,88, S;12-18, U;80,82, Y80.

\subsubsection{Methods}

The number of new injuries presenting to each GP in the AMTS survey was expressed as a rate per 100 encounters. The variation of these rates among the GPs was assumed to have a Poisson distribution as injuries account for less than $5 \%$ of the total doctorpatient encounters. Mean rates for each Australian state and standard deviations were calculated by fitting a Poisson model in EGRET (SERCS 1989). The sampling fraction of the survey was calculated by dividing the total number of encounters registered in the study by the total number of GP encounters in the country over the period of the study. The number of GP items of service in Australia during the study period was supplied by the Health Insurance Commission. An estimate of the injury incidence for 1990-1991 was obtained by multiplying the sample means and standard deviations by the inverse of the sampling fraction.

Costs for the AMTS study sample were calculated by apportioning GP management at recorded encounters into its component parts and identifying costs for each portion. An estimate of the cost of injury for the whole of Australia in 1990-91 was obtained by weighting the calculated sample costs by the estimated national incidence of injury.

General practice management of injury involves the following components:

i. Consultation and treatments provided at the time of consultation

ii. Radiology ordered

iii. Medications prescribed

iv. Arrangement of follow up 
v. Referred treatment (medical specialists, hospital admissions, physiotherapists, other)

(i) Consultation (including special procedures)

The cost of GP consultations and treatments was derived from the Medicare Benefit Schedule Book and Supplement 1st November 1990 (Commonwealth Department of Community Services and Health 1990a). Medicare is a scheme which pays benefits for professional services to patients provided by private medical practitioners. GPs identify the professional services rendered by item numbers to which schedule fees are attached. Usually, general practitioners charge for an item number denoting a patient consultation of a particular duration. Instead, he or she may charge for the treatments provided if these treatments fall within specifications of the Medicare guidelines. In certain circumstances both a consultation item number and a special procedural item number may be charged. The AMTS survey form recorded only one Medicare item number per encounter irrespective of the number of problems treated at the encounter and the number of items charged to Medicare. It was not possible to maintain direct links between each problem treated at an encounter and the item number charged. The AMTS did directly link the treatments provided to each problem managed during the encounter.

For encounters where injury was the only condition managed, the recorded item number was wholly attributable to the injury condition. It was assumed that the difference between the distribution of item numbers for this group of patients and the group of people who had more than one condition managed at the encounter was due to the added management of the other conditions. The cost of treating the injury component of the mixed consultations was assumed to be the same as for the injuryonly group. Those injury-related encounters for which no item number was available were assigned the mean cost derived from the available item numbers. 
Those treatments provided by the GPs at the time of the consultation which attracted Medicare benefits were converted to costs by assigning appropriate item numbers. These treatment costs were then added to the cost estimates derived from the recorded item numbers to obtain an estimate of the total costs attributable to consultations.

Medications prescribed

All medications prescribed for treatment of a new injury were identified in the AMTS database and converted to an appropriate cost. The cost of drugs on the Pharmaceutical Benefits Schedule (PBS) (Commonwealth Department of Health Housing and Community Services 1990b) was taken as the PBS "Dispensed Price for Maximum Quantity" (Commonwealth Department of Health Housing and Community Services 1992) current at 1st November 1990. Drugs which were identified in the AMTS only by their class name and not generic name were ascribed the mean cost for those in the survey of that class, as were the relatively small number of non-PBS drugs in the survey. Wound dressings and bandages are readily available and can be purchased without prescription. Those wound dressings prescribed by GPs in the survey were not considered to represent the cost of these materials adequately and were excluded from the general practice injury-cost estimates.

\section{(iii) Radiology performed}

There were no direct links in the AMTS database between radiology ordered and any single problem managed at an encounter. Radiology costs were calculated by identifying the injuries of those people on whom radiology was ordered and taking the Medicare Schedule Fee for radiology to that body-part. Those people for whom radiographs were ordered but whose recorded injury condition was non-specific for body part were assumed to have the same distribution of radiology item numbers as the remainder of the sample. If the other conditions treated in those patients on whom radiology was ordered were of a kind where radiographs were appropriate, it was assumed the radiological investigations were for the non-injury condition. 
The number of encounters at which a new injury was managed and where the patient was advised to return for follow-up consultation were identified. As there was no indication in the database whether the arranged follow-up appointment was for the injury or the other conditions treated at that encounter, it was assumed that injury and non-injury conditions were the cause of the follow-up appointments in the same ratio as they were present in the initial consultation. Calculations were made on the basis that those who were advised to return did so but that no further ongoing consultations were required (ie the cost of only one follow-up consultation was included). These consultations were assigned the item number corresponding to a Medicare standard short consultation.

\section{(v) Referral treatment}

Specialist consultations arising from referrals were not considered to fall within the defined GP management and so were not included in the cost estimates. Similarly, hospital admissions were also excluded. Although GPs in some country areas do have admitting rights to hospitals, these arrangements were not considered sufficiently common to negate the rule that, in general, admission to hospital moves the patient outside the sphere of GP care.

Referrals to other health professionals were considered to be part of the general practice management of the patient. Physiotherapy constitutes the bulk of this referred treatment. Although the number of sessions required for an acute injury varies considerably depending upon the severity and type of injury, three visits were taken as the minimum requirement. The cost of physiotherapy were calculated on the basis that each referral to a physiotherapist resulted in three sessions (one first visit and two standard consultations). These value of these was obtained from Australian College of Physiotherapists advised fee schedule for the 1st November 1990. Other health 
professional referrals were few in number with unquantifiable service provision and hence were not included in the cost estimates.

\subsubsection{Results}

(i) Consultation (including special procedures)

There were 98,796 encounters in the AMTS database. There were 4,950 encounters at which a new injury was one of the problems managed. During the period in which this data was collected the Health Insurance Commission received claims for $85,242,365$ general practice items of service. Thus, by extrapolation, in the 12 month period from October 1990 to October 1991 there were 4,266,000 (95\% CI 4,005,000-4,545,00) new injuries which received primary medical care in general practice in Australia. The age and sex distributions of the people who presented with these injuries are described in Figure 9.1.

Figure 9.1 Incidence of injuries presenting to general practice as place of first treatment by age and sex

\section{Injuries per 100 people}

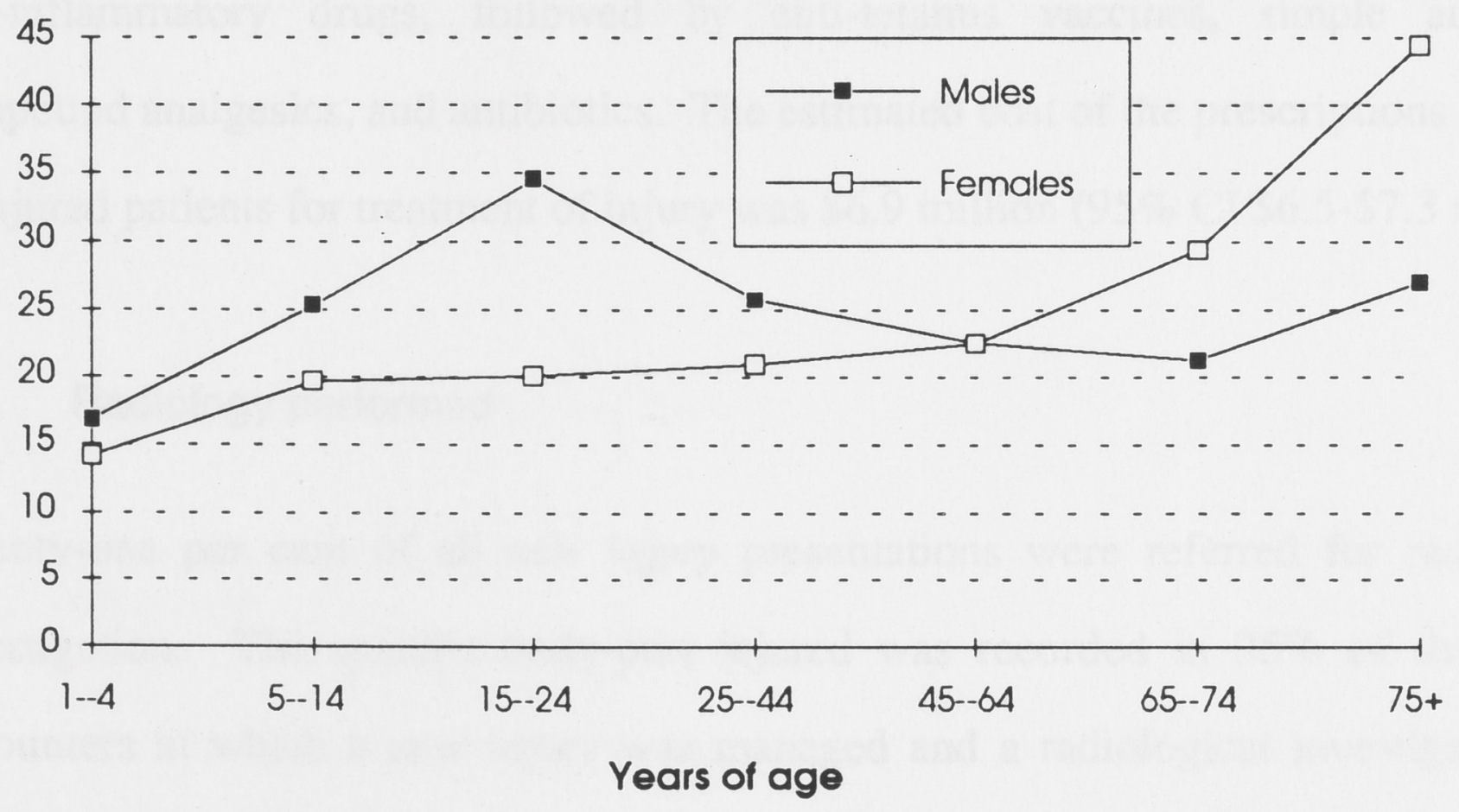


For $70 \%$ of all encounters in which a new injury was one of the problems managed, the injury was the only problem managed. Item numbers were available for $88 \%$ of these encounters and the mean schedule fee was $\$ 19.40$. This mean cost was ascribed to the $12 \%$ of injury consults without item numbers. Thus the national cost for consultations for injuries was $\$ 92.5$ million (95\% CI $\$ 87-\$ 99$ million).

Fifty-one percent of encounters involved therapeutic procedures, including bandaging, strapping and dressings and closure of lacerations. Twenty-five percent of patients received advice and reassurance. The additional cost of procedures performed was calculated at \$5.5 million (95\% CI \$5-\$6 million).

The injury-related cost of all consultations was estimated to be the sum of the consultation costs and procedure costs, that is $\$ 98$ million (95\% CI $\$ 92-\$ 104$ million).

\section{(ii) Medications prescribed}

After excluding over-the-counter dressings and non-injury related medications there were an average of 18 prescription drugs ordered by GPs per 100 new-injury encounters. Eight-two percent of these were for specified PBS drugs. The most frequently prescribed injury-related medications for this sample were non-steroidal anti-inflammatory drugs, followed by anti-tetanus vaccines, simple analgesics, compound analgesics, and antibiotics. The estimated cost of the prescriptions provided to injured patients for treatment of injury was $\$ 6.9$ million (95\% CI \$6.5-\$7.3 million).

\section{(iii) Radiology performed}

Twenty-one per cent of all new injury presentations were referred for radiological investigation. The specific body-part injured was recorded in $35 \%$ of the AMTS encounters at which a new injury was managed and a radiological investigation was ordered. Less than $5 \%$ of these encounters also involved a non-injury condition for which radiology may have been appropriate. The cost of radiology for acute injuries in general practice was estimated to be $\$ 29.5$ million (95\% CI $\$ 28-\$ 31$ million). 
In $46 \%$ of encounters a follow-up consultation was arranged. The cost for this component of the treatment was estimated to be $\$ 28$ million (95\% CI $\$ 26.5-\$ 30$ million).

\section{(v) Referrals}

Only $1.3 \%$ of the patients who presented to general practice for first treatment of a new injury were referred for hospital admission, only $3.6 \%$ were referred to specialist surgeons and $4.7 \%$ percent were referred to non-medical health professionals. Eightytwo percent of non-medical specialist referrals were to physiotherapists. Costs for this component were \$15 million (95\% CI \$14-\$6 million).

Cost of general practice injury in the ACT

In 1990-91, the estimated total cost of managing acute injury in general practice in Australia was $\$ 178$ million (95\% CI $\$ 167-\$ 190$ million) at a mean cost of per injury of $\$ 42$. This represents a cost of $\$ 43$ in 1992 using an estimated inflation factor of $2.4 \%$ (AIHW, 1993). From estimates obtained in Chapter Five, the incidence of injury that was treated in general practice in the ACT in 1992 was 23,500 (95\% CI 17,00032,000 ) Thus the estimated total cost of injury in the ACT was approximately $\$ 1$ million.

\subsection{Emergency department study}

\subsubsection{Methods}

The cost of acute management of minor injury in adults presenting to emergency departments in the ACT during 1992 was calculated in a manner similar to that described for the general practice component of the study. First it was noted that management of injury in hospital emergency departments involves the following 
components; consultation and treatments provided at the time of consultation, radiology ordered, medications prescribed, arrangement of follow up and referred treatment. The estimated number of injury consultations, the number and type of radiological investigations undergone and the number and type of special treatments performed were obtained from samples of injured patients treated in the emergency departments in the ACT. The total costs were estimated by ascribing the relevant Medicare item number to each of these components and summing the corresponding Medical Benefit Schedule (MBS) fees. These totals were inflated to population estimates by multiplying by the inverse of the sampling fraction. Mean cost per injury was calculated by dividing total costs by the number of non-admitted injuries treated in the ACT emergency departments during 1992.

There were two samples used in the estimation of these costs. The first sample was used to estimate the incidence and distribution of non-hospitalised emergency department injury in the ACT as in Chapter Five. Briefly, beginning on the 20th January 1992 the emergency department register was examined every eighth day for 12 months. The medical records of all patients whose presenting problem was a possible injury or poisoning were examined and relevant details recorded where the diagnosis was first presentation injury of an ACT resident. Total numbers of injuries in the ACT of each type were estimated by multiplying by the inverse of the sampling fraction.

The second sample was used to identify the proportion of injuries to each body part which underwent radiological investigation. This sample was the subsample of the follow-up study group described in detail in Chapter Five. This group included a sample of patients, 16 years and over, who presented to ACT emergency departments between 12 March and 12 May 1993 who responded to the ACTISPP survey and registered for the further follow-up study. The medical records of the non-admitted subjects in this sample were drawn and any radiological investigations were noted. The 
sample was divided into strata according to body-part injured. The proportion of injuries in each strata that underwent radiological investigation was noted. These proportions were assumed to reflect the proportions of people radiographed for each body-part group in the total emergency department treated, non-admitted population. The total number of each type of radiological investigation was then calculated from the injury incidence and distribution data.

Medicare items numbers were ascribed to the components of the emergency department injury management as outlined below.

(i) Consultation and treatments provided at the time of consultation

The item number for an emergency department doctor consultation was taken as that for a standard hospital consultation of more than five minutes duration but not more than twenty five minutes duration. In 1992 the scheduled fee for this item number was $\$ 21.00$. The item numbers for each extra treatment provided at the time of consultation were included when they fell within the MBS descriptions of chargeable procedures. The procedures for which these extra costs were chargeable included treatment of fractures, management of dislocations, suturing of lacerations and removal of foreign bodies. The MBS item number for these treatments depends upon the nature of the injuries and the body part involved and were identified in the study accordingly.

\section{(ii) Radiology ordered}

Investigations conducted during the management of acute injury in emergency departments were essentially radiological. Pre-testing demonstrated that for nonadmitted patients few pathology tests were ordered. Appropriate Medicare items numbers and the corresponding scheduled fee were identified for each radiological test ordered. 
The cost of dressings and medications in treatments identified by an Medicare item number are included in the Medicare Schedule Fee structure. PBS prescriptions written for injured patients treated in the emergency department are not reliably recorded on the medical records and were therefore not included in the cost estimates.

(iv) and (v) Follow-up and referred treatment

Except in special circumstances (eg: incomplete assessment or deteriorating condition), follow-up of injured patients in the ACT does not occur in the emergency department. Patients needing review are referred directly to specialists or asked to return to their general practitioner for further care if needed. These referrals were not considered to fall within the defined acute emergency department management and so were not included in the cost estimates.

\subsubsection{Results}

There were 2,866 subjects in the incidence and distribution sample who were treated in the emergency department for injury and not admitted to hospital. There were 841 ambulatory subjects in the second sample used to identify the radiology costs. The sample used for identifying radiological investigations performed was not truly representative of the study population (Table 9.1). Of the 841 injured subjects identified in sample two, radiology history could not be found for $92(11 \%)$. Radiological investigations were performed on $55 \%$ of the remainder. 
Table 9.1 Distribution of incidence sample and radiology sample by sex, injury type and body-part injured (percent of sample in brackets).

\begin{tabular}{lcrcr}
\hline & $\begin{array}{c}\text { Incidence sample } \\
(n=2,866)\end{array}$ & $(\%)$ & $\begin{array}{c}\text { Radiology sample } \\
(n=841)\end{array}$ & $(\%)$ \\
\hline Sex \# & 1835 & $(64)$ & 563 & $(67)$ \\
Male & 1028 & $(36)$ & 278 & $(33)$ \\
$\quad$ Female & 3 & $(0)$ & & \\
$\quad$ Missing & & & & \\
Injury Type * & 901 & $(31)$ & 219 & $(26)$ \\
Lacerations & 1415 & $(49)$ & 487 & $(58)$ \\
Soft tissue injury & 369 & $(13)$ & 117 & $(14)$ \\
Fractures & 181 & $(6)$ & 18 & $(2)$ \\
Other & 143 & $(5)$ & 17 & $(2)$ \\
Body-part injured * & 627 & $(22)$ & 125 & $(15)$ \\
Systemic injury & 1101 & $(38)$ & 322 & $(38)$ \\
Head and face & 752 & $(26)$ & 316 & $(38)$ \\
Upper limb & 243 & $(9)$ & 61 & $(7)$ \\
Lower limb & & & & \\
Other & & & & \\
\hline
\end{tabular}

$\# X^{2} p>0.05,{ }^{*} x^{2} p<0.001$, percents may not add to 100 because of rounding

There were an estimated $24,000(23,000-25,000)$ ambulatory injuries treated in ACT emergency departments during 1992. Attendance costs for this group were $\$ 481,500$. The costs of radiological investigations on this group were $\$ 522,500$. The cost of procedural treatments over and above consultation costs were $\$ 495,000$. The total cost of treating these injuries was approximately $\$ 1.5$ million with a mean cost per injury of $\$ 65$. The average cost of treating injury in emergency departments was $\$ 22$ more per injury than the cost of treatment in general practice. The combined emergency department and general practice cost of managing acute injury in 1992 for the ACT population of 296,000 people was about $\$ 2.5$ million.

\subsection{Discussion}

The methods used in the study to isolate the emergency department costs of treating injury presentations resulted in an estimate of $\$ 65$ which was $\$ 16$ less than the average emergency department patient costs estimated by the AIHW. The common cost schedule used for the emergency department and GP components of the study standardised the unit costs and enabled comparisons to be made between the cost of 
emergency department and GP treatment of injury. It is possible that the less costly treatment of injury in general practice is a product of different GP treatment practices but more probably reflects a different patient casemix. In Chapter Five it was noted that emergency department patient and GP patients differed in terms of nature of injury and body-part injured. It would appear that patients with lacerations and fractures who require more costly treatments tend to present to emergency departments rather than general practitioners. The higher rate of radiological investigation in the emergency department patients and the greater proportion of procedural treatments involved in emergency department care noted in this chapter could be a product of those patient differences and may have accounted for the increased cost.

The estimate for the costs of radiology for emergency department patients in this study needs to be considered with some caution, as the sample used to establish this figure was not representative of the incident population. The radiology follow-up group included only those people aged 16 years or older. It is unclear whether this would have resulted in an under- or over-estimate of the numbers of radiological tests ordered, but, as radiology is usually ordered on the basis of the condition rather than on the age of the patient, the effect of the age difference in the radiology sample on the estimates would probably not have been great. Differences between the two groups by nature of injury and body part injured would not have affected the estimates because the sample was stratified by these variables in the analysis. Differences in these three variables suggest however, that the two samples may have differed in other ways for which they were not controlled and differential biases may have been introduced at this point.

As has been noted in criticisms of cost-of-illness studies (Shiell et al. 1987) there are limits to the usefulness of a calculated "bottom line" cost. For the estimated cost figure of nearly $\$ 2.5$ million to assist the decision making process it needs to be considered in relation to other results. Health development personnel, for example, might wish to compare the injury costs with the cost of other conditions in order to help set priorities for action. Health administrators might note the apparent savings to be made by 
encouraging the public to seek general practice rather than emergency department care of their injuries. However, the incidence-based cost estimates calculated in this chapter can also provide useful information to assist the formulation of strategies for injury control. Incidence-based cost estimates provide a basis for estimating the cost savings which might result from the implementation of appropriate injury prevention programmes.

Clearly, the estimates presented in this chapter understate the total cost of minor injury in the community. Only immediate costs of treating acute injury were estimated and no attempt was made to account for the ongoing costs of treating chronic injuries. The general practice estimates do not include the cost of general practice follow-up of injuries first treated in an emergency department. No attempt was made to account for indirect or on-costs of the minor injuries. However, what this chapter does provide is a minimum estimate of the economic cost of minor injury in the community. Even this minimum estimate quite clearly establishes minor injury as having an major impact on the health resources of the community. 


\section{Chapter Ten}

\section{The Minor Injury Scale}

\subsection{Introduction}

In Chapters Eight and Nine the importance of the contribution made by non-lifethreatening injuries to the community burden arising from injury was demonstrated. Further application of epidemiology to the control of these injuries requires their categorisation on a severity scale (Chapter One). Existing injury scores however, were developed as aids to trauma management or as markers of potentially fatal injuries (States and Viano 1990) and are therefore not suitable for minor injury.

To categorise injury properly on the basis of its public health impact, one would need to use a score based upon a property that encapsulated that aspect of injury that made it a public health problem. The score would need to discriminate well throughout the entire range of that property, would need to be free from any covert information bias and would have to be demonstrably valid and reliable. Although there have been several useful developments in this area, such a score does not yet exist and the lack of such a score has impeded research into non-hospitalised injury. This chapter presents a two-dimensional injury scoring system that recognises both the injury severity and the cumulative morbidity experienced over the period of time till the injury's recovery. The Minor Injury Scale tabulates a set of injury severity scores and morbidity scores for non-life-threatening injury that can be used in conjunction with the Abbreviated Injury Scale to expand the available discrimination at the lower end of the severity range. 


\subsection{Methods}

\subsubsection{Study design}

This chapter uses data from the prospective cohort study described in Chapter Eight. It was conducted using a sample of injured people to identify the health outcomes arising from the range of injuries in the study population. The representativeness of the sample was confirmed by comparing its characteristics with those of a criterion sample which was drawn from the same population. The methods of measuring the health outcomes were chosen so as to equate injury-related health-status immediately after injury occurrence' with injury severity and 'the total quantity of health loss accumulated over time until recovery' with injury morbidity. The new injury severity and morbidity scores were constructed by taking the summary outcome values for each injury type.

\subsubsection{Population}

The study was conducted in the Australian Capital Territory (ACT) between 12 March 1992 and 12 March 1993. The study population included ACT residents, 16 years or older, who received primary care for a new injury in an ACT hospital emergency department during 1992.

\subsubsection{Process and instruments}

Two samples were drawn from the study population, an incidence and distribution sample (Chapter Five) and a follow-up sample (Chapter Eight). The incidence sample was drawn from the emergency departments of the ACT and was used to confirm the representativeness of the follow-up sample. The follow-up sample was used to generate the data from which the Minor Injury Scale was derived (Chapter Eight). Injury severity is defined as the 'injury-related health-status index value immediately after injury occurrence' (or 'initial health value') as measured by the first administration of the HCIQ (Figure 8.1). Injury morbidity is the accumulated health loss due to injury 
over the time until recovery and was represented by the area under the health loss by time curve (Figure 8.1).

\subsubsection{Analysis}

The characteristics of the follow-up sample were compared with the incidence sample with regard to age, sex, postcode of residence, nature of injury, body-part injured and whether the patient was hospitalised. The statistical significance for each of the differences was assessed using Chi-squared statistics. There was sufficient power in the study to identify statistical significance for two percent differences with a beta value of 0.2 .

Those subjects in the follow-up sample who had sustained a single injury were selected and categorised into groups according to their AIS numeric identifier. The median health value obtained at the time of first presentation was calculated for each injury type. This value was deemed to be the Minor Injury Severity Score (MISS) for each injury defined by the relevant AIS identifier. Thus the severity scores, being derived from injury-related health indexes immediately after the injuries occurrence, range between zero, for injuries that usually result in death, to one, for those injuries which produce no change in a persons health state.

Correction factors to be applied to the severity scores to allow for the influence of age and sex were obtained from a regression model in which these demographic variables were regressed against the initial health value while controlling for nature of injury and body part injured. Further multivariate regression models were used to explore the effect of pre-existing disease and multiple injury's on the injury health values.

After selecting those subjects who had sustained only one injury, the follow-up sample was divided into groups according to the AIS numerical identifiers and the injury morbidity was calculated for each injury. The median value for each injury type was deemed to be the Minor Injury Morbidity Score (MIMS) for the injury defined by the relevant AIS identifier. In contradistinction to the MISS which was derived from a 
health index, the MIMS was based on health loss and so high morbidity scores indicate a more serious injury than low morbidity scores. Correction factors for the MIMS for age and sex were obtained in a similar manner to those for the MISS.

The Minor Injury Scale (MIS) is the tabulation of the MISS and MIMS for the full range of injuries listed by AIS numerical identifier.

\subsection{Results}

\subsubsection{Reporting bias and variable distribution}

Satisfactory medical records for 2,144 subjects were obtained in the emergency department incidence sample, representing $95 \%$ of the eligible emergency department attendances. There were 2,823 adult ACT residents registered by ACTISPP during the study period who had given their consent for involvement in further studies. The inclusion bias of this ACTISPP sample has been presented in detail in Chapter Seven.

Of the 2,823 ACTISPP subjects approached, 1,396 agreed to participate in the followup study. As discussed in Chapter Eight, the respondents were more likely to be younger and female than the non-respondents. Of the respondents there were 953 subjects who were successfully followed until their injury had recovered or their censorship at six months. There were no significant differences between the group that completed follow-up and those that dropped out.

Those subjects who completed follow-up and whose data was used for the derivation of the Minor Injury Scale were compared with those of the incidence sample. There was no statistical difference between the groups by age $(p=0.15)$ but statistically significant differences were present at the $p<0.001$ level for sex, nature of injury, bodypart injured and whether admitted. The characteristics of the samples are presented in Table 10.2 
Table 10.1 Distribution of samples by age, sex, nature of injury, body-part injured and whether admitted (percent in brackets).

\begin{tabular}{|c|c|c|c|c|c|c|}
\hline & \multicolumn{2}{|c|}{$\begin{array}{c}\text { Emergency } \\
\text { department incidence } \\
\text { sample }\end{array}$} & \multicolumn{4}{|c|}{ Follow-up sample } \\
\hline & \multirow[b]{2}{*}{$n=2144$} & \multirow[b]{2}{*}{$(\%)$} & \multicolumn{2}{|c|}{ Registered } & \multicolumn{2}{|c|}{ Completed follow-up } \\
\hline & & & $n=1396$ & $(\%)$ & $n=953$ & $(\%)$ \\
\hline \multicolumn{7}{|l|}{ Age(in years) } \\
\hline $16-24$ & 867 & $(40)$ & 525 & (38) & 347 & (36) \\
\hline $25-34$ & 551 & (26) & 373 & (27) & 247 & (26) \\
\hline $35-44$ & 312 & (15) & 224 & (16) & 169 & (18) \\
\hline $45-54$ & 194 & (9) & 148 & (11) & 108 & (11) \\
\hline $55+$ & 220 & (10) & 126 & (9) & 82 & (9) \\
\hline \multicolumn{7}{|l|}{ Sex } \\
\hline Male & 1407 & (66) & 821 & (59) & 557 & (58) \\
\hline Female & 733 & (34) & 527 & (38) & 390 & (41) \\
\hline Missing & 4 & $(0)$ & 48 & (3) & 6 & (1) \\
\hline \multicolumn{7}{|l|}{ Nature of injury } \\
\hline Lacerations & 684 & (32) & 359 & (26) & 292 & (31) \\
\hline Sprains & 1061 & (50) & 800 & (57) & 513 & (54) \\
\hline Fractures & 266 & (12) & 207 & (15) & 129 & (14) \\
\hline Other & 133 & (6) & 30 & (2) & 19 & (2) \\
\hline \multicolumn{7}{|l|}{ Body-part injured } \\
\hline Systemic & 105 & (5) & 29 & (2) & 18 & (2) \\
\hline Head/Face & 443 & (21) & 222 & (16) & 173 & (18) \\
\hline Upper limb & 772 & (36) & 507 & (36) & 346 & (36) \\
\hline Lower limb & 614 & (29) & 544 & (39) & 350 & (37) \\
\hline Trunk & 183 & (9) & 90 & (6) & 62 & (7) \\
\hline Other & 20 & (1) & 4 & (0) & 4 & (0) \\
\hline Missing & 7 & (0) & & & & \\
\hline \multicolumn{7}{|l|}{ Admitted } \\
\hline Yes & 106 & (5) & 32 & (2) & 20 & (2) \\
\hline No & 2034 & (95) & 1364 & (98) & 933 & (98) \\
\hline
\end{tabular}

Percents may not add up to 100 because of rounding to nearest whole percent

\subsubsection{Scale development}

The 397 subjects who were registered in the follow-up sample between 12 March and 11 May 1992 received questions about pre-existing conditions and 147 of the subjects described a condition of some kind. Linear multivariate regression was used to evaluate the effect of the dichotomous variable 'presence of pre-existing condition' on the severity values of the subjects in the subsample. After controlling for age, sex, nature of injury, body-part injured and whether multiple injuries, subjects with pre- 
existing conditions were found to have an initial health value that was 0.070 lower than those without, but this effect was not significant $(p=0.13)$.

One hundred and thirty-four subjects who were registered in the complete follow-up sample had sustained multiple injuries. Linear multivariate analysis performed on this total sample indicated that the presence of a second injury associated with the major injury treated decreased the subject's initial health value by $0.100(p<0.001)$. In addition being female decreased the initial health value by $0.090(\mathrm{p}<0.001)$, and age increased the value by 0.050 for each increase in age group $16-35,35-55,55+$ $(p=0.04)$. After excluding those subjects in the sample who had sustained more than one injury, there were 46 discrete AIS injury types which occurred on four or more occasions in the sample. The initial health value ranged from 0.422 to 1.000 and were well distributed between these extremes.

With regard to the long term morbidity caused by injury, the subsample registered between 12 March and 11 May was analysed using linear multivariate regression to identify the effect of pre-existing conditions. After controlling for nature of injury, body part injured and multiple injuries, presence of pre-existing conditions did not significantly affect the morbidity values $(\mathrm{p}=0.27)$.

The effect of the presence of more than one injury on the injury morbidity was calculated by including this variable as an effect variable in a regression model with age and sex as controls and the morbidity value as the outcome variable. For the total sample the presence of a second acute injury at presentation increased the morbidity value by $0.080(\mathrm{p}=0.01)$. In addition, being female increased the morbidity value by $0.080(\mathrm{p}=0.01)$ but age had no significant effect $(\mathrm{p}=0.79)$.

Amongst those single injury patients who completed the follow-up process, there were 39 different injury types with four or more occurrences. The morbidity score was calculated as the median morbidity value for each injury type. The resulting morbidity scores were found to be well distributed over a range from 0 to 0.25 . 
The MISS and MIMS for three upper limb injury types and the comparable lower limb injuries are presented in Table 10.2. For both upper and lower limb injuries, sprains have, on average, a more serious MISS than lacerations, and fractures have a more serious MISS than sprains. The differences between these injuries is more pronounced when their MIMS are considered. Injuries to the lower limb result in more serious outcome (both MISS and MIMS) than their upper limb counterparts.

Table 10.2 Extract from the Minor Injury Scale listing the Minor Injury Severity Score (MISS) and the Minor Injury Morbidity Score (MIMS) for six injury types identified by Abbreviated Injury Scale (AIS) codes. The number of study subjects with each injury type (n) and the quartile ranges of the outcome values are also listed.

\begin{tabular}{lccccccc}
\hline Injury type & AIS code & MISS & $(n)$ & Quartiles & MIMS & $(n)$ & Quartiles \\
\hline lacerated arm & 710602.1 & 0.792 & $(122)$ & $(0.580-0.939)$ & 0.004 & $(96)$ & $(0.001-0.014)$ \\
sprained wrist & 751420.1 & 0.518 & $(19)$ & $(0.473-0.563)$ & 0.026 & $(13)$ & $(0.010-0.167)$ \\
fractured radius & 752800.2 & 0.501 & $(30)$ & $(0.448-0.555)$ & 0.040 & $(20)$ & $(0.015-0.146)$ \\
lacerated leg & 810602.1 & 0.580 & $(22)$ & $(0.504-0.582)$ & 0.017 & $(17)$ & $(0.004-0.036)$ \\
sprained ankle & 850206.1 & 0.501 & $(206)$ & $(0.456-0.563)$ & 0.031 & $(134)$ & $(0.012-0.065)$ \\
fractured tibia & 853404.2 & 0.422 & $(26)$ & $(0.422-0.503)$ & 0.101 & $(18)$ & $(0.058-0.153)$ \\
\hline
\end{tabular}

The full Minor Injury Scale (MIS) is shown in Table 10.3. Because the MISS was derived from an injury-related health index, the lower values represent lower predicted initial health values and hence indicate the more serious injuries. In contradistinction, the MIMS was based on accumulated health loss values and so higher values indicate the more serious injuries. 
Table 10.3 Minor Injury Scale

\begin{tabular}{|c|c|c|c|}
\hline Injury Type * & $\begin{array}{l}\text { AIS identifier } \\
\text { and score }\end{array}$ & $\begin{array}{l}\text { Minor Injury } \\
\text { Severity Score }\end{array}$ & $\begin{array}{l}\text { Minor Injury } \\
\text { Morbidity Score }\end{array}$ \\
\hline Unconsciousness $<1 \mathrm{hr}$ & 160202.2 & 0.510 & 0.004 \\
\hline Contusion, skin, face & 210402.1 & 0.542 & 0.018 \\
\hline Minor, laceration, skin, face & 210602.1 & 0.743 & 0.005 \\
\hline NFS, eye & 240499.1 & 0.708 & 0.003 \\
\hline NFS, fracture, nose & 251000.1 & 0.682 & 0.015 \\
\hline Contusion, skin, neck & 310402.1 & 0.516 & 0.018 \\
\hline Contusion, ribcage, thorax & 450202.1 & 0.563 & 0.005 \\
\hline NFS, fracture, ribcage, thorax & 450210.1 & 0.518 & 0.027 \\
\hline Contusion, skin, abdomen & 510402.1 & 0.501 & 0.023 \\
\hline Strain, cervical spine & 640278.1 & 0.563 & 0.012 \\
\hline Strain, thoracic spine & 640478.1 & 0.610 & -- \\
\hline Strain, lumbar spine & 640678.1 & 0.501 & 0.021 \\
\hline Abrasion, skin, upper ex. & 710202.1 & 0.939 & 0.001 \\
\hline Contusion, skin, upper ex. & 710402.1 & 0.631 & 0.010 \\
\hline Minor, laceration, skin, upper ex. & 710602.1 & 0.792 & 0.004 \\
\hline Minor, penet. inj. skin, upper ex. & 716002.1 & 0.728 & 0.002 \\
\hline Sprain, Carp/Metacarp. upper ex. & 750402.1 & 0.583 & 0.007 \\
\hline Contusion, elbow joint, upper ex. & 750610.1 & 0.729 & -- \\
\hline Sprain, elbow joint, upper ex. & 750620.1 & 0.560 & -- \\
\hline Dislocation, interphal. upper ex. & 750800.1 & 0.593 & -- \\
\hline Contusion, shoulder, upper ex. & 751010.1 & 0.518 & 0.027 \\
\hline Sprain, shoulder, upper ex. & 751020.1 & 0.518 & 0.024 \\
\hline Dislocation, shoulder, upper ex. & 751030.2 & 0.475 & 0.022 \\
\hline Contusion, wrist, upper ex. & 751410.1 & 0.640 & 0.021 \\
\hline Sprain, wrist, upper ex. & 751420.1 & 0.518 & 0.026 \\
\hline Fracture, carp/metacarp, upper ex. & 752002.2 & 0.492 & 0.034 \\
\hline Fracture, clavicle, upper ex. & 752200.2 & 0.422 & 0.076 \\
\hline Fracture, finger, upper ex. & 752404.1 & 0.570 & 0.030 \\
\hline Crush, finger, upper ex. & 752406.2 & 0.668 & 0.009 \\
\hline Fracture NFS, radius, upper ex. & 752800.2 & 0.500 & 0.040 \\
\hline Minor, penet. inj. skin, lower ex. & 816002.1 & 0.701 & 0.003 \\
\hline Abrasion, skin, lower ex. & 810202.1 & 0.558 & 0.011 \\
\hline Contusion, skin, lower ex. & 810402.1 & 0.518 & 0.017 \\
\hline Minor, laceration, skin, lower ex. & 810602.1 & 0.580 & 0.017 \\
\hline Sprain, ankle, lower ex. & 850206.1 & 0.501 & 0.031 \\
\hline Sprain, foot, lower ex. & 850404.1 & 0.510 & 0.021 \\
\hline Contusion, hip, lower ex. & 850602.1 & 0.563 & -- \\
\hline Contusion, knee, lower ex. & 850802.1 & 0.501 & 0.044 \\
\hline Sprain, knee. lower ex. & 850826.2 & 0.501 & 0.099 \\
\hline Sprain, metatarsal, lower ex. & 851002.1 & 0.529 & 0.019 \\
\hline Fracture, NFS, foot, lower ex. & 852000.2 & 0.501 & .- \\
\hline Fracture, metatarsal, lower ex. & 852200.2 & 0.476 & 0.035 \\
\hline Contusion, tibia, lower ex. & 853402.1 & 0.613 & -- \\
\hline Fracture NFS, tibia, lower ex. & 853404.2 & 0.422 & 0.101 \\
\hline Fracture, toe, lower ex. & 853602.1 & 0.515 & 0.033 \\
\hline NFS, Burn & 912000.1 & 0.515 & 0.014 \\
\hline
\end{tabular}

*Abbreviated Injury Scale codes 
To ensure the MIMS was not affected by a premature censoring of unrecovered injuries, the MIMS was recalculated using extrapolated estimated life-time health loss for those subjects unrecovered at six months. A Kaplan-Meier survival curve was plotted for the recovery of the subjects in the sample and it was found to closely approximate the exponential model $S(t)=0.087+e^{-0.205 t}$. It reaches a minimum-value asymptote of 0.087 as time approaches infinity. Recalculating the MIMS using lifetime morbidity based upon a non-recovery rate of $8.7 \%$ of the sample did not affect the MIMS values obtained.

\subsection{Discussion}

This chapter presents an account of a new score based upon health loss due to injury by which non-fatal injuries can be grouped and compared for the purposes of refining strategies for injury control. Injury scoring systems facilitate the epidemiological study of injury by categorising the components of the injury problem according to a relevant property. The injury severity scales that are currently most widely used are "likelihood of death" scores. The public health impact of minor injury, however, cannot be measured by these instruments. In 1992, Alexander et al argued for the development of a severity score specifically for minor injuries, noting that:

at present, no scales have been developed for categorisation of these relatively less serious, non-life threatening injuries in either adults or children (Alexander et al, 1992:148).

The MIS may fill this gap. It is a valid and practical scale for use in both clinical and research environments and has been shown to discriminate well across the range of injury types commonly experienced in population-based injury studies. The face validity of the MIS is confirmed by its compliance with clinical expectations: fractures score more severely than sprains, and sprains score more severely than cuts and lower limb injuries score more severely than upper limb injuries. It is possible to rank all injuries on the scale, with full health and death anchoring the scale's limits, so that although the scale is most useful in the range of minor injury the values of the minor injury scores can be seen in context of the whole picture. 
The MIS can be used to predict the amount of health loss due to particular injuries or groups of injuries. The health loss can be refined using weights for age and sex. Further, the morbidity scores can be summed to produce massed outcome totals for aggregated injuries. By multiplying the incidences of given injury types by their MIMS, the predicted community morbidity, measured in quality adjusted life years, of that injury type can be estimated. It is possible, for example, to compare the total quality adjusted life years lost due to head injuries with the total quality-adjusted life years lost from lacerations, sprains and fractures.

The empirical approach to the development of the MIS which ensured the variety of its applications described above is, however, also responsible for some of the limitations of the scoring system. The final sample used to calculate the MIS constituted only $30 \%$ of those people initially approached. Comparison of the distribution of study variables between the final sample and the more rigorously-selected incidence sample identified small but statistically significant differences and differential biases may have been introduced in at this point. For example, the MIS scores may have been inflated by an over-sampling of people with exaggerated health interest.

A second issue relating to the methodology of the MIS development is the use of a quality-of-life based outcome measure. Though there have been some studies where the outcomes of injury have been measured in terms of quality of life it is not common practice to do so. It should be recognised, however, that current injury outcome measures are not conceptually different from the quality of life instrument used in this study (Section 2.4.3).

The study described in this chapter represents a first step in the development of a new epidemiological tool for injury control research. Because the scale was developed empirically from a small representative sample of injuries presenting to emergency departments in the ACT it can categorise only those injuries which occur commonly in the community. Further studies using the MIS will need to be conducted to expand the number of injury types covered by the scale. Repeating the process with larger samples 
would extend the number of injury types scored. Repeating the process in other samples would also complete the validation of the scale. The MIS was developed in a sample of injured subjects and needs to be tested in other samples to confirm its predictive powers. This preliminary evaluation indicates that further work would be justified. 


\section{Chapter Eleven}

\section{The public health impact of minor injury}

\subsection{Introduction}

There is no question that injury is a public health problem of major importance. However, as discussed in the first three chapters, much of the public health impact of injury remains ill-defined. In particular the public health impact of minor injury is unknown. The seven interdependent studies described in this thesis were conducted in an effort to partially redress this gap in current knowledge.

\subsection{Summary of thesis}

The importance of the general practice component of the injury problem was described in Chapter Four. Injured patients presenting to general practice tended to be older, non-Australian and non-European born, and more often employed than the group of injured patients attending hospital emergency departments. There was no difference in the sex distribution between injured patients presenting to general practice and emergency departments. The place of most recent accident for those people who presented to emergency departments was significantly different from those who presented to general : practice. About $31 \%$ of all injuries seen in emergency departments occurred in places of sport and recreation while only $19 \%$ of those attending general practices reported this as the place of the most recent accident.

Chapter Five described the incidence and distribution of injury in an Australian population. It was shown that $48,500(95 \%$ CI $41,000-56,000)$ injuries requiring medical attention were sustained by residents in the Australian Capital Territory from January 1992 to January 1993. This represents 164 (95\% CI 139 - 189) injuries per 1,000 inhabitants per year. General practice was the chosen place of presentation for 
primary care in approximately one half of the cases. Estimates of the incidence dimensions of the injury iceberg are presented in Figure 11.1.

Figure 11.1 The injury iceberg: percent of total medically-treated injuries in the ACT during 1992 by category

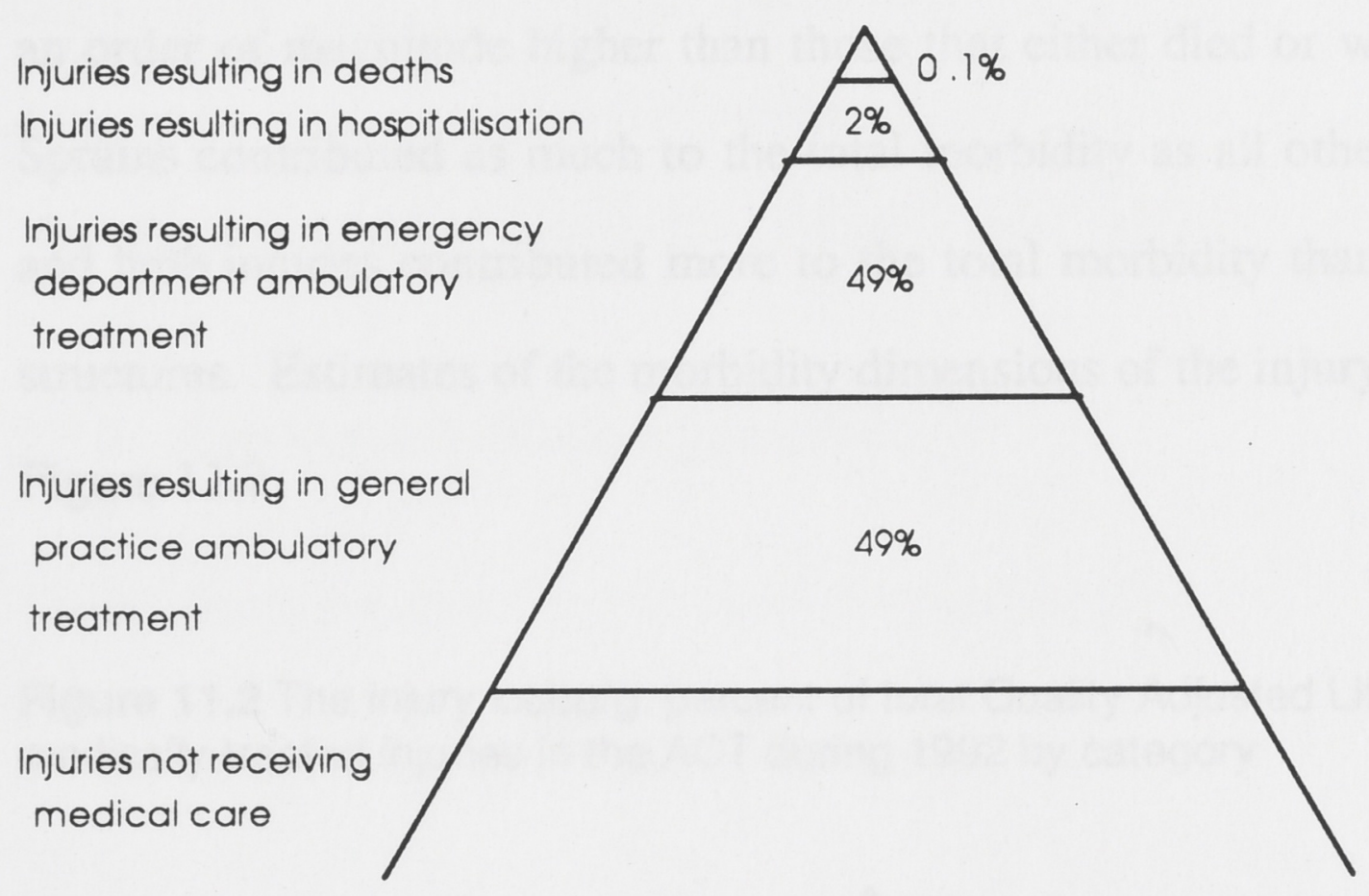

Chapter Six provided an account of the Australian Capital Territory Injury Surveillance and Prevention Programme. The sensitivity of the surveillance system was $31 \%$ (95\% CI $30 \%-32 \%$ ). The distribution of the ACTISPP sample did not differ from the expected distribution for sex or socio-economic status $(p>0.05)$. It differed by age only in the $5-14$ age group $(\mathrm{p}<0.001)$. With respect to the nature of injury and body part injured, the difference was statistically significant $(\mathrm{p}<0.001)$ but was no greater that $7 \%$ for any of the categories. Two percent of the surveillance sample were admitted to hospital compared with an estimated four percent of the target population $(\mathrm{p}<0.001)$.

Chapter Seven described the development of the Health Consequences of Injury Questionnaire (HCIQ). The HCIQ is a simple questionnaire designed for selfcompletion that identifies a subject's injury-related health profile. The preliminary studies described showed the HCIQ to be practical and reliable. When scored with QWB weights the HCIQ can be validly used to predict QWB health index values. 
The study described in Chapter Eight showed that minor injuries were responsible for the greater part of the injury-related health burden in the adult community. Injuries coded as AIS $>1$ account for $20 \%$ of the morbidity arising over the first six months after injury and about $25 \%$ of the estimated lifetime morbidity. The lifetime estimates of morbidity due to injuries not considered serious enough to admit to hospital were of an order of magnitude higher than those that either died or were admitted to hospital. Sprains contributed as much to the total morbidity as all other injury types combined, and limb injuries contributed more to the total morbidity than injuries to more central structures. Estimates of the morbidity dimensions of the injury iceberg are presented in Figure 11.2

Figure 11.2 The injury iceberg: percent of total Quality Adjusted Life Years lost from medically-treated injuries in the ACT during 1992 by category

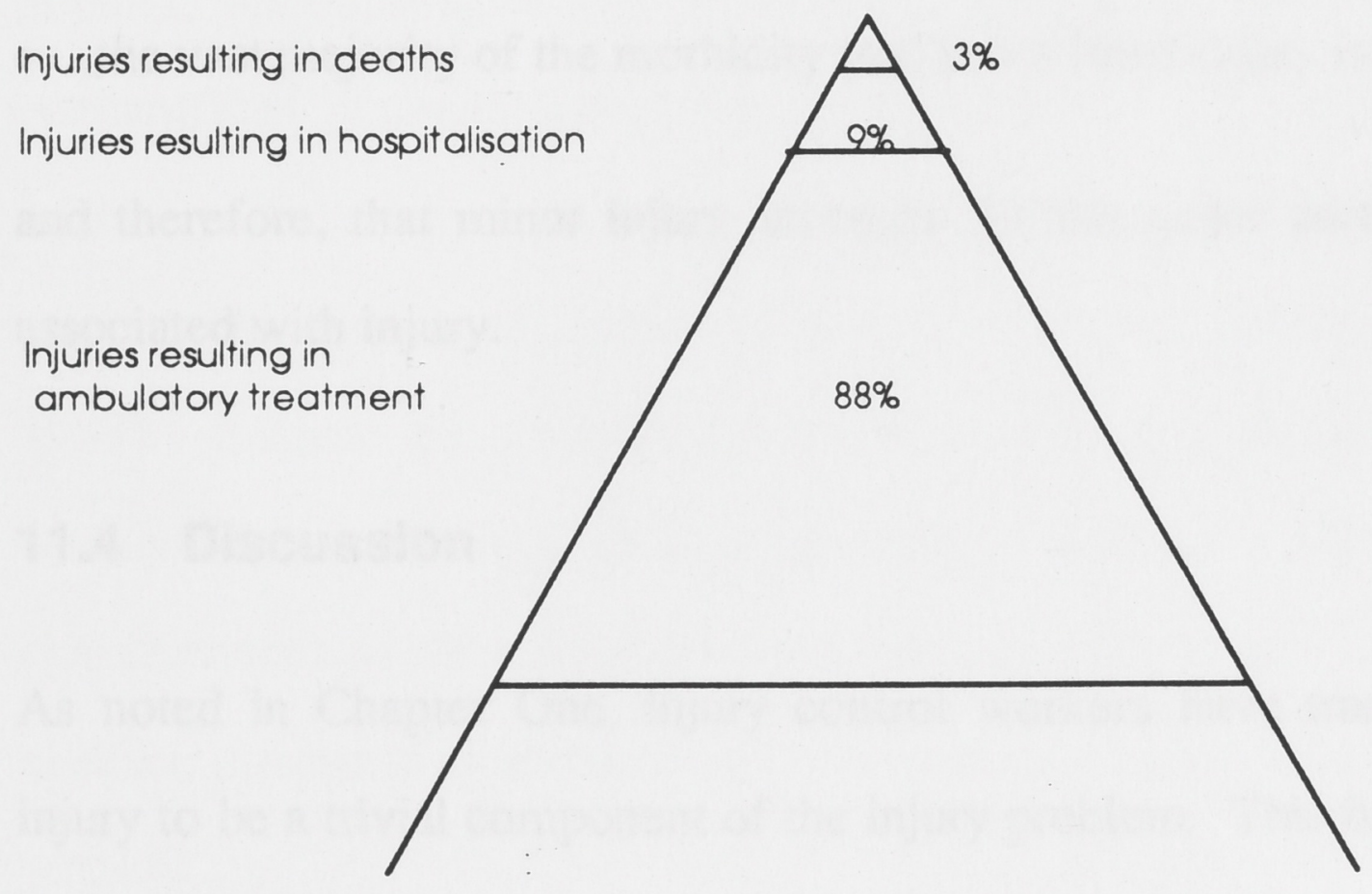

The results presented in Chapter Nine show that minor injuries were responsible for substantial economic costs to a community. The total cost of treating ambulatory injuries in the emergency departments of ACT during 1992 was estimated to be approximately $\$ 1.5$ million at a mean cost per injury of $\$ 65$. The estimated average cost of treating injury in emergency departments was $\$ 22$ more per injury than the cost of treatment in general practice. The total community direct cost of managing acute injury in 1992 for the ACT population of 296,000 people was about $\$ 2.5$ million. To 
the extent that the financial and medical resources dedicated to minor injury are unavailable to spend on other sectors of health care, these results clearly establish minor injury as an important public health problem.

Chapter Ten presented a preliminary account of a new score based upon health loss due to injury. Using this scale, non-fatal injuries can be grouped and compared for the purposes of refining strategies for injury control. The strengths of the new scoring system are its theoretical coherence, its practicability, and its validity.

\subsection{Evaluation of the hypothesis}

The evidence presented in this thesis demonstrates that:

- the vast majority of all injuries are minor,

- the vast majority of the morbidity that arises from injury is due to minor injury,

and therefore, that minor injury accounts for the major part of public health burden associated with injury.

\subsection{Discussion}

As noted in Chapter One, injury control workers have traditionally believed minor injury to be a trivial component of the injury problem. This belief is illustrated by such comments as:

The large number of children who suffer from trivial accidental injuries and attend our emergency departments tend to hide the causes of the less frequent, but more severe injuries. This may lead to misdirected efforts at prevention (Hurst and Ellis 1977:768).,

and:

Let the medical profession speak out on these preventable causes of serious injuries to children and not waste its time on emergency department trivia (Hurst and Ellis 1977:770). 
More recently, however, several researchers have drawn attention to the importance of minor injury. Examples of this change of emphasis include:

The most interesting result that has evolved from this study is the large percentage of minor and moderate extremity injured patients who are still convalescing at 1 year post discharge (Mackenzie et al. 1988:294).,

and:

Another misconception that needs to be considered is the presumption that the preponderance of disability is to be found among persons who have been hospitalised (Waller et al. 1990:359).

There are several explanations for the past tendency to trivialise minor injury. First, it is intuitively preferable to save a life than prevent a sprained ankle. While there are still people dying from injury, it is difficult to spare resources for dealing with less catastrophic events.

Second, historically, people with an interest in injury research have largely been concentrated in teaching hospitals (Section 2.3). In hospital-based studies, severely injured patients are over-represented in the study samples, and hence they overestimate the amount of severe injury in a population (Waller 1988; Payne and Waller 1989). Indeed, the referral procedures in Australia ensure that severe injuries are usually over-represented in teaching hospital samples. Attempting to generalise the characteristics of these samples leads to such unlikely conclusions as "25\% of injured children ....require specialised trauma centre care as expeditiously as possible" (Taylor 1989:6).

Third, the universal use of 'threat-to-life' injury severity scores renders the outcomes of non-hospitalised injuries invisible. Injury severity scores were originally developed as aids to trauma management or markers of potentially fatal injuries. Although the majority of injuries scored by these scores receive their minor status only with regards to their chances of precipitating death, these injuries become regarded as minor in the more general sense; that is, inconsequential and therefore not a significant contributor to the injury problem. 
Finally, the lack of appreciation of the prevention paradox (Rose 1981:1850) contributes to the disregard for minor injury control as an individual's gain from the prevention of a minor injury is often felt to be not sufficient to make the prevention effort worthwhile.

A genuine application of the public-health model to the problem of injury needs to be built around contemporary definitions of health and mass approaches to injury control. Emphasis must be placed upon addressing the causes, not of high individual morbidity, but of high population morbidity. The development of instruments capable of measuring the health outcomes of minor injury will make these outcomes 'epidemiologically visible'. Population-based identification of cases is needed to ensure that minor injuries are better represented in study samples.

The work presented in this thesis involved population-based studies of injury using quality-of-life outcome measures. It is this methodology which enabled the relative importance of minor injury to be evaluated. At a population level, the sum of the morbidity of the ubiquitous minor injuries far outweighed the combined health losses from the more serious injuries.

In accordance with the basic public health principle that small changes in the health of large numbers can produce substantial health benefits for the community, minor injury should now be addressed as a community health problem of vital importance. 


\section{Bibliography}

AHMAC/NH\&MRC working groups. Better health outcomes for Australians. (Draft Report) Canberra: Department for Human Services and Health, 1994a.

AHMAC/NH\&MRC working groups. Better health outcomes for Australians: national goals, targets and strategies for injury prevention and control. (Draft Report) Canberra: Department for Human Services and Health, 1994b.

Alexander BH, Rivara FP, Thompson D, Thompson RS. Measurement of severity for non-hospitalised injuries in the paediatric age group. Pediatr Emerg Care 1992;8:14851.

American Public Health Association. Suggested home accident prevention activities for health departments. Am J Public Health 1956;46:625-30.

Anderson JP, Bush JW, Berry CC. Internal Consistency analysis: a method for studying the accuracy of function assessment for health outcome and quality of life evaluation. $J$ Clin Epidemiol 1988;41:127-37.

Anderson JP, Kaplan RM, Berry CC, Bush JW, Rumbaut RG. Interday reliability of function assessment for a health status measure. Med Care 1989;27:1076-84.

Andreassand DC. A framework for costing accidents and accident types. Accid Anal Prev 1985; 17:111-7.

Anonymous. Assessment of disability [editorial] Lancet 1986;1:591-592.

Atkins AS. The economic and social costs of road accidents in Australia 1978. Melbourne: Centre for Environmental Studies, University of Melbourne, 1981.

Australian Bureau of Statistics. 1989-90 National Health Survey: Accidents, Australia. Cat. No. 4384.0. Canberra: ABS, 1992.

Australian Bureau of Statistics. Deaths Australia. Canberra: ABS, 1993.

Australian Institute of Health and Welfare. Australia's Health 1990. Canberra: AGPS, 1990.

Australian Institute of Health and Welfare. Australia's Health 1992. Canberra: AGPS, 1992.

Australian Institute of Health and Welfare. Health expenditure Bulletin No 8, AIHW, 1993.

Baker SP, O'Neill B, Haddon W, Long WB, The Injury Severity Score: a method for describing patients with multiple injuries and evaluating emergency medical care. $J$ Trauma 1974;14:187-96.

Baker SP, O'Neill B, Karpf RS. The Injury Fact Book. Lexington, Mass.: Lexington Books, 1984. 
Balla JI, Moraitis S. Knights in armour. A follow-up study of injuries after legal settlement. Med J Aust 1970;2:355-61.

Barancik JI, Chatterjee BF, Green YC, Michenzi EM, Fife D. Northeastern Ohio Trauma Study: I. Magnitude of the problem. Am J Public Health 1983;73:746-51.

Barry PZ. Individual versus community orientation in the prevention of injuries. Prev Med 1975;4:47-56.

Baumgartner L, Ramsey E. Johann Peter Frank and his 'System einer vollstandigen medicinischen Polizey'. Ann Med Hist 1933;5:525-32.

Better Health Commission. Better Health Commission. Vol. 2. Looking forward to better health: the task-forces and working groups. Canberra: AGPS,1986;192-235.

Bland JM, Altman DG. Statistical methods for assessing agreement between two methods of clinical measurement. Lancet 1986;1:307-10.

Brewin CR, Robson MJ, Shapiro DA. Social and psychological determinants of recovery from industrial injuries. Injury 1983;14:451-5.

Bridges-Webb C, Britt H, Miles DA, Neary S, Charles J, Traynor V. The Survey of Morbidity and Treatment in General Practice 1990-91. Med J Aust 1992;157:S1-57.

Brooks, RG. Development and construction of health status measures. The Swedish Institute for Health Economics Report 4. 1986

Bull JP. Disabilities caused by road traffic accidents and their relation to severity scores. Accid Anal Prev 1985;17:387-97.

Bureau of Transport and Communications Economics. Social costs of transport accidents in Australia. Canberra: AGPS, 1992.

Burke DC, Burley HT, Ungar GH. Data on spinal injuries - Part II. outcome of the treatment of 352 consecutive admissions. Aust N Z J Surg 1985;55:377-82.

Cain CM, Ryan GA, Fraser R, Potter G, McLean AJ, McCaul K, Simpson DA. Cervical spine injuries in road traffic crashes in South Australia, 1981-86. Aust NZ Surg 1989;59:15-9.

Carr-Hill RA, Morris J. Current practice in obtaining the "Q" in QALYs: a cautionary note. BMJ 1991;303:699-701.

Centers for Disease Control. Estimated years of potential life lost before age 65 and cause-specific mortality by cause of death: US 1985: public health issues in the use of years of potential life lost. Morbid Mortal Weekly Rep 1987;36:447.

Chalmers DJ, Cecchi J, Langley-JD, Silva PA. Injuries in the 12th and 13th years of life. Aust J Paediatr 1989;25:14-20.

Champion HR, Copes WS, Sacco WJ. Lawnick MM, Bain LW, Gann DS, Gennarelli T, MacKenzie EJ, Schwaitzberg S. A new characterization of injury severity. J Trauma 1990;30:539-46. 
Chan BS, Walker HPJ, Cass BSH. Urban Trauma: An Analysis of 1,116 Paediatric Cases. J Trauma 1989;29:1540-7.

Chapman AL. Accident Prevention. Halsey MN, ed. New York: McGraw-Hill, 1961.

Cochran WG. Sampling Techniques. New York: John Wiley and Sons, 1977.

Colditz GA. The Australian Health Survey: implications of reported accidents for medical education. Med J Aust 1983;1:125-7.

Collopy BT, Tulloh BR, Rennie GC, Fink RLW, Rush JH, Trinca GW. Correlation between injury severity scores and subjective ratings of injury severity: A basis for trauma audit. Injury 1992;23:489-93.

Committee on Injury Scaling. The Abbreviated Injury Scale, 1990 revision. Des Plaines, IL.: Association for the Advancement of Automotive Medicine, 1990.

Committee on Injury Scaling. Injury Impairment Scale. Des Plaines, IL.: Association for the Advancement of Automotive Medicine, 1994.

Committee on Trauma Research. Injury in America: a continuing public health problem. Washington, DC., National Academy Press, 1985.

Commonwealth Department of Community Services and Health. Medicare Benefits Schedule book 1990. Canberra: AGPS, 1990a.

Commonwealth Department of Community Services and Health. Pharmaceutical Benefits schedule. Canberra: AGPS, $1990 \mathrm{~b}$.

Commonwealth Department of Community Services and Health. Medicare Benefits Schedule book 1992. Canberra: AGPS, 1992.

Commonwealth Department of Health Housing and Community Services. Manual of resource items and their associated costs. Canberra: AGPS, 1992

Consumer Product Safety Commission. Final report on the evaluation and calibration of NEISS. Washington, DC: CPSC,1978.

Cornes P, Bochel HM; Aitken RCB. Rehabilitation and return to work of employers' liability claimants. Int J Rehab Research 1986;9:119-28.

Cornes P, Bochel HM. Fitness for work: Medicolegal assessment of residual disability and employment handicap. Int J Rehab Research 1987;10 Suppl 5:S309-17.

Coulter A. Measuring morbidity. BMJ 1987;294:263.

Day LM, Ozanne-Smith J. Epidemiology of injury in a Regional Centre of Victoria. 13th Scientific Meeting of the International Epidemiological Association, Sydney: AEA, 1993

Day RJ, Vinen J, Hewitt-Falls E. Major trauma outcomes in the elderly. Med J Aust 1994;160:675-8. 
De Haven H. Mechanical analysis of survival in falls from heights of fifty to one hundred and fifty feet. War Med 1942;2:586-96.

Department of Health and Human Services. Vital Statistics of the United States 1989. DHHS Publication No. 92-1102.

Eisen M, Donald CA, Ware JE, Brook RH. Conceptualisation and measurement of health for children in the Health Insurance Study. Publication R-2313-HEW. Santa Monica, CA.: Rand Corporation, 1980.

Elechi EN, Etawo T. Pilot study of injured patients seen in the University of Port Harcourt teaching hospital, Nigeria. Injury 1990;21:234-8.

Erickson P, Kendall EA, Anderson JP, Kaplan RM. Using composite health status measures to assess the nation's health. Med Care 1989;27:S66-76.

Fanshel S, Bush JW. A Health Status Index and its application to health services outcomes. Operations Res 1970;18:1021-6.

Fife D, Barancik JI, Chatterjee-BF. Northeastern Ohio Trauma Study: II. Injury rates by age, sex, and cause. Am J Public Health 1984;74:473-8.

Finkler SA. The distinction between costs and charges. Ann Intern Med 1982;96:1029.

Ford B. Head injuries what happens to survivors? Med J Aust 1976;1: 603-5.

Forward G. Indoor cricket injuries. Med J Aust 1988;148:560-1.

Frutiger A, Ryf C, Bilat C, Rosso R, Furrer M, Cantieni R, Ruedi T, Leutenegger A. Five years' follow-up of severely injured ICU patients. J Trauma 1991;31:1216-25.

Gable CB. A compendium of public health data sources. Am $J$ Epidemiol 1990;131:381-93.

Gallagher SS, Finison K, Guyer B, Goodenough S. The incidence of injuries among 87,000 Massachusetts children and adolescents: Results of the 1980-81 Statewide Childhood Injury Prevention Program Surveillance System. Am J Public Health 1984;74:1340-1347.

Ganiats TG, Palinkas LA, Kaplan RM. Comparison of Quality of Well-Being Scale and Functional Status Index in patients with Atrial Fibrillation. Med Care 1992;30:958-64.

Gibson JJ. The contribution of experimental psychology to the formulation of the problem of safety: A brief for basic science. In: Behavioural approaches to accident research. New York: Association for the Aid of Crippled Children, 1961;77-89.

Giles JM. Hospital inpatient accident costs for road traffic accident casualties in Western Australia, 1988. Road Accident Prevention Research Unit, Department of Medicine, University of Western Australia. 1988 
Ginpil S, Schneider R, Stone S. Years of potential life lost through road crashes: 1990. a comparison with other causes of death. Federal Office of Road Safety, Canberra: 1992.

Glancy KE, Glancy CJ, Lucke JF, Mahurin K, Rhodes M, Tinkoff GH. A study of recovery in trauma patients. J Trauma 1992;33:602-9.

Gofin R, Israeli I, Palti H.. The incidence of childhood and adolescent injuries and their outcome: a population-based study. Israel J Med Science 1991;27:566-71.

Gordon D, Silverstone H. Patterns of accident morbidity in a sub-tropical city. Med J Aust 1965;Aug 21:316-21.

Gordon J. The epidemiology of accidents. Am J Public Health 1949;39:504-15.

Graitcer PL. The development of state and local injury surveillance systems. J Safety Research 1987;18:191-8.

Gudex C, Kind P. The QALY toolkit. Discussion paper 38. Center for Health Economics, University of York, 1987.

Guria JC. Length of hospitalisation-an indicator of social costs of disabilities from traffic injuries. Accid Anal Prev 1990;22:379-89.

Guria JC. The expected loss of life quality from traffic injuries requiring hospitalisation. Accid Anal Prev 1993;25:765-72.

Gustafson DH, Fryback DG, Rose JH, Prokop CT, Detmer DE, Rossmeissl JC, Taylor CM, Alemi F, Carnazzo AJ. An evaluation of multiple trauma severity indices created by different index development strategies. Med Care 1983;21:674-91.

Haddon W Jr. A note concerning accident theory and research with special reference to motor vehicle accidents. Ann N Y Acad Science 1963;107: 635-46.

Haddon W Jr, Suchmann E, Klein. Accident research: methods and approaches. New York: Harper and Row, 1964.

Haddon, W Jr. The basic strategies for preventing hazards of all kinds. Hazard Prev 1980a; 16:8-12.

Haddon W Jr. Advances in the epidemiology of injuries as a basis for public policy. Public Health Rep 1980b;95:411-21.

Harlan LC, Harlan WR, Parsons PE. The economic impact of injuries: A major source of medical costs. Am J Public Health 1990;80:453-9.

Harrison JE, Frommer MS, et al. Deaths as a result of work-related injury in Australia, 1982-1984. Med J Aust. 1989;150:118-25.

Harrison JE. Nature and dimensions of injury in Australia. In: Monash University Short course on injury research and prevention. Melbourne: Monash University Accident Research Centre, 1991. 
Harrison JE, Tyson D. Injury surveillance in Australia. Acta Paediatr Jpn $1993 ; 35: 171-8$.

Health Ministers' Forum. Towards a National Health Policy: a discussion paper. Canberra: AGPS, 1994.

Hildingsson C, Toolanen G. Outcome after soft tissue injury of the cervical spine. Acta Orthop Scand 1990;61:357-9.

Hirsch AE, Eppinger RH. Impairment scaling from the Abbreviated Injury Scale. In: Proceedings of the 28th conference of the American Association for Automotive Medicine Conference. Des Plaines, IL.: AAAM, 1984:209-24.

Holbrook TL, Hoyt DB, Anderson JP, Hollingsworth-Fridlund P, Shackford SR. Functional limitation after major trauma: a more sensitive assessment using the Quality of Well-Being scale-the trauma recovery pilot project. J Trauma 1994;36:74-8.

Hurst J, Ellis M. Fatal accidents to children: Implications for prevention. Med J Aust $1977 ; 2: 768-70$.

Jamieson KG, Wigglesworth EC. The dimensions of the accident problem in Australia. Aust N Z J Surg 1977;47:135-8.

Jansson BR, Jacobsson BS. Medical consequences of work-related accidents on 2,454 Swedish farms. Scand J Work Environ Health 1988;14:21-6.

Jennett, B. Outcome after severe head injury: Definitions and predictions. Med J Aust 1976;2:475-7.

Johns AM. Time off work after hand injury. Injury 1981;12:417-24.

Jones NR, Blumbergs PC, North JB. Acute subdural haematomas: aetiology, pathology and outcome. Aust N Z J Surg1986;56:907-13.

Kalsbeek WD, Bowling JM, Morris PD. Choosing criteria to screen for specific medical conditions from non specific sources: an application to injury surveillance. Am J Epidemiol 1992;135:553-63.

Kaplan RM, Bush JW, Berry CC. Health status: types of validity and the Index of Well-being. Health Serv Res 1976;11:478-507.

Kaplan RM, Bush JW, Berry CC. The reliability, stability and generalisability of a health status index. American Statistical Association, proceedings of the social statistics section 1978;704-9.

Kaplan RM, Bush JW. Health-related quality of life measurement for evaluation research and policy analysis. Health Psychology 1982;1:61-80.

Kaplan RM, Anderson JP, Erickson P. Estimating well-years of life for a new public health indicator. Proceedings of the 1989 Public Health Conference on Records and Statistics. National centre for health statistics. DHHS Publication Number (PHS) 901214;1990:302. 
Kaplan RM. Health outcome models for policy analysis. Health Psychology 1989;8:723-35.

Katz S. Assessing self maintenance: Activities of daily living, mobility and instrumental activities of daily living. J Am Geriatrics Soc 1983;31:721-7

Kirkwood BR. Essentials of medical statistics. Oxford: Blackwood Publications, 1988.

Kirwin JR, Chaput de Saintonge DM, Joyce CRB, Curry HLF. Clinical Judgement analysis-practical application in Rheumatoid Arthritis. British $J$ Rheumatol 1983;22:S18-23.

Kraus J. Robertson LS. Injuries and public health. In: Public Health and Preventive Medicine. Last JM, Wallace RB; eds.. Connecticut: Appleton and Lance,1992;102134.

Lamberts H. Woods ME. International classification of primary care. Oxford: Oxford University Press, 1987.

Lanese RR, Strauss RH, et al. Injury and disability in matched men's and women's intercollegiate sports. Am J Public Health 1980;80:1459-62.

Langley JD. The need to discontinue the use of the term "accident" when referring to unintentional injury events. Accid Anal Prev 1988;20:1-8.

Langley JD, McLoughlin E. Injury mortality and morbidity in New Zealand. Accid Anal Prev 1989;21:243-54.

Last JM. A Dictionary of Epidemiology. (2nd ed.) Oxford: Oxford University Press, 1988.

Lawton MP, Moss M, Fulconer M, Kleban MH. A research and service oriented multilevel assessment instrument. J Gerontology 1982;37:91-9

Lee RH. Needs for rehabilitation after minor fractures. Int $J$ Rehab Research 1982;5:378-80.

Leeder S. A Faculty of Public Health Medicine for Australia. Med J Aust 1991;155:7.

Lehman AF, Prostrado LT, Rachuba LT. Convergent validation of quality of life assessments for persons with severe mental illnesses. Qual Life Res 1993;2:327-33.

Leininger BE, Gramling SE, Farrell AD, Kreutzer JS, Peck EA. Neuropsychological deficits in symptomatic minor head injury patients after concussion and mild concussion. J Neurol Neurosurg Psychiatry 1990:53:293-6.

Levine RS. A review of long-term effects of selected lower limb injuries. SAE Technical Paper \#860501. Warrandale PA: Society of Automotive Engineers, 1987.

Liang MH, Jette AM. Measuring functional ability in chronic arthritis: A critical review. Arthritis and Rheumatism 1981;24:80-6. 
Linqvist KS. Epidemiology of accidents in a Swedish Municipality. Accid Anal Prev 1989;21:33-43.

Lodge JF, Langley JD, Begg DJ. Injuries in the 14th and 15th years of life. J Paediatr Child Health 1990;26:316-322.

London P. Some observations on the course of events after severe injury of the head. Ann Royal College Surg 1967;41:460-79.

Lopez W, Rennie T. A Survey of accidents to children aged under 15 years seen at a district hospital in Sydney in one year. Med J Aust 1969;1:806-9.

Lovell S, Kibble J, Tennent P, Zabell K, Dugdale AE. The functioning of a children's hospital casualty department. Med J Aust 1975;1:135-8.

Lyle, DM. Quine S Pierce JP, Thompson PC. Outcome following physical trauma: a comparative approach. Int J Disab Stud 1990;12:113-8

MacKenzie EJ, Shapiro S, Moody M, Siegel JH, Smith RT. Predicting posttrauma functional disability for individuals without severe brain injury. Med Care 1986;24: 377-87.

MacKenzie EJ, Shapiro S, Smith RT, Siegel JH, Moody M. Factors influencing return to work following hospitalisation for traumatic injury. Am $J$ Public Health 1987;77:329-34.

MacKenzie EJ, Seigel JH, Shapiro S, Moody M, Smith RT. Functional Recovery and Medical Costs of Trauma: An analysis by type and severity of injury. $J$ Trauma 1988;28:281-97.

MacKenzie EJ, Shapiro S, Siegel JH. The economic impact of traumatic injuries. JAMA 1988;260:3290-6.

MacKenzie EJ, Morris JA, Edelstein S. Effect of pre-existing disease on length of hospital stay in trauma patients. J Trauma 1989;29:757-64.

MacKenzie EJ, Morris JA, Smith GS, Fahey M. Acute hospital costs of trauma in the United states: Implications for regionalised systems of care. J Trauma 1990;30:1096101.

Maurette P, Masson F, Nicaud V, Cazaugade M, Garros B, Tiret L, Thicoipe M, Emy P. Posttraumatic disablement: a prospective study of impairment, disability, and handicap. J Trauma 1992;35:728-36.

McLennan W. Socio-economic indexes for areas, Catalogue No. 1356.0. Canberra: ABS, 1990.

Miller T, Viner J, Rossman S, Pindus N, Gellert W, Douglass J. The costs of highway crashes. Washington, DC: The Urban Institute. 1991.

Miller TR, Luchter S, Brinkman CP. Crash costs and investment. Accid Anal Prev 1989;21:303-15. 
Miller TR, Pindus NM, Douglass JB. Medically related motor vehicle injury costs by body region and severity. J Trauma 1993;34:270-5.

Miller TR, Pindus NM, Leon NM, Douglass JB. Motor vehicle injury costs by body region and severity. AAAM 34th Annual Proceedings, Scottsdale, Arizona, USA, AAAM, 1990.

Miller TR. Costs and functional consequences of U.S. roadway crashes. Accid Anal Prev 1993;25:593-607.

Milzman DP, Boulanger BR, Rodriguez A, Soderstrom CA, Mitchell KA, Magnant CA. Pre-existing disease in trauma patients: A predictor of fate independent of age and injury severity score. J Trauma 1992;32:236-43.

Minaire P. Disease, illness and health: theoretical models of the disablement process. Bulletin of the World Health Organization 1992;70:373-9.

Mock CN, Adzotor KE, Conklin E, Denno DM, Jurkovich GJ. Trauma outcomes in the developing world: comparison with an urban level 1 trauma centre. J Trauma 1993;35:518-23.

Morris JA, Sanchez AA, Bass SM, MacKenzie EJ. Trauma patients return to productivity. J Trauma 1991;31:827-34.

Nagel DA, States JD, Dashboard and bumper-knee - will arthritis develop? 21st Conference of the American Association for Automotive Medicine, Des Plaines, IL., AAAM. 1977:272-9.

National Committee for Injury Prevention and Control. Injury Prevention: Meeting the Challenge. New York: Oxford University Press, 1989.

National Highway Safety Administration. Functional Capacity Index. Federal Register 1992;57:13157-65.

National Injury Surveillance Unit. ISIS Injury Research Information System. Adelaide: NISU, 1992.

Nolan T, Penny M. Epidemiology of non-intentional injuries in an Australian urban region: Results from injury surveillance. J Paediatr Child Health 1992;28:27-35.

Nutbeam D, Wise M, Bauman A, Harris E, Leeder S. Goals and Targets for Australia's Health in the Year 2000 and Beyond. Canberra: AGPS, 1993.

O'Connor PJ. Accidental death in Australian children. Aust Paediatr J 1983;19:230-2.

Ozanne-Smith J, Brumen I. Shire of Bulla "safe living program" - overview of injuries presenting to General Practitioners: pilot study. Monash University Accident Research Centre. Melbourne: MUARC. 1992

Payne SR, Waller JA. Trauma registry and Trauma Centre biases in injury research. $J$ Trauma 1989;29:424-9.

Pearn JH, Nixon J. The Canberra Child Trauma Study. Med J Aust 1981;2:612-3. 
Pennie B, Agambar L. Patterns of injury and recovery in whiplash. Injury 1991;22:579.

Philadelphia Injury Prevention Program. Philadelphia Injury Prevention Program: progress report and program plan. Philadelphia, Philadelphia Department of Health. 1988.

Rhodes M, Aronson J, Moerkirk G. Petrash E. Quality of life after the trauma centre. $J$ Trauma 1988;28:931-8.

Rice DP, MacKenzie EJ, and Associates. Cost of illness in the United States: A report to Congress. San Francisco, CA: Institute for Health and Aging, University of California and injury prevention Center, The Johns Hopkins University, 1989.

Rimel RW, Giordani B, Barth JT, Boll TJ, Jane JA. Disability caused by minor head injury. Neurosurgery 1981;9:221-8.

Ring IT, Berry G, Dan NG, Mandryk JA, North JB, Selecki BR, Sewell MF, Simpson DA, Stenning WA, Vanderfield GK. Epidemiology and clinical outcomes of neurotrauma in New South Wales. Aust N Z J Surg 1986;56:557-66.

Rivara FP, Calonge N, Thompson RS. Population-Based study of unintentional injury incidence and impact during childhood. Am J Public Health 1989;79:990-4.

Rivara FP, Thompson RS, Thompson DC, Calonge N. Injuries to children and adolescents: impact on physical health. Pediatrics 1991;88:783-8.

Robertson LS. Injury Epidemiology. Oxford: Oxford University Press, 1992.

Rockett IRH, Smith GS, Homicide, suicide, motor vehicle crash, and fall mortality: United States experience in comparative perspective. Am J Public Health. 1989a;79:1396-400.

Rockett IRH, Smith GS. Injuries and the Australian mortality mosaic: a comparison with the United Kingdom and New Zealand. Public Health 1989b;103:353-61.

Rose G. Strategy of prevention: lessons from cardiovascular disease. $B M J$ 1981;282:1847-51.

Rose, G. Sick individuals and sick populations. Int J Epidemiol 1985;14:32-8.

Ross SE, O'Malley KF, Stein S, Spettell CM, Young G. Abbreviated injury scaling of head injury as a prognostic tool for functional outcome. Accid Anal Prev 1992;24:1815 .

Rosser RM, Watts VC. The measurement of hospital output. Int $J$ Epidemiol 1972;1:361-8.

Rowe IL, Accidents, Poisoning and Violence. In: The Australian General Practice Morbidity and Prescribing Survey 1969 to 1974. Med J Aust 1976;2:S22-3.

Rutledge R, Fakhry S, Baker C, Oller D. Injury severity grading in trauma patients: A simplified technique based upon ICD-9 coding. J Trauma 1993;35:497-507. 
Ryan GA. Improving head protection for cyclists, motorcyclists and car occupants. World J Surg 1992;16:398-402.

Sahlin Y, Stene TM, Lereim I, Balstad P. Occurrence of injuries in a defined population. Injury 1990;21:155-7.

Saltman D, Man A. General Practice databases in Australia. Med J Aust 1992;156:1620.

Schelp L, Svanstrom L. A model for registration and mapping of accident cases in health care. Scand J Prim Health Care 1987;5:91-9.

Schelp L. Epidemiology as a basis for evaluation of a community intervention programme on accidents. Sundbyberg: Karolinska Institute, 1987.

Schwartz S, Richardson, J, Glasziou PP. Quality-adjusted life years: origins, measurements, applications, objections. Aust J Public Health 1993;17:272-8.

Scotford N, Lawson JS. Trauma: The nature of the problem in a Sydney suburban population. Med J Aust 1977;1:249-51.

Shiell A, Gerard K, Donaldson C. Cost of illness studies: an aid to decision making? Health Policy 1987;8:317-23.

Shiell A, Smith RD. A tentative cost-utility analysis of road safety education. Aust $J$ Public Health 1993; 17:128-30.

Silva JF. Review of patients with multiple injuries treated at University Hospital Kuala Lumpur. J Trauma 1984;24:526-31.

Simpson D, North B, Gilligan J, McLean J, Woodward A, Antonio J, Altree P. Neurological injuries in South Australia: the influence of distance on management and outcome. Aust N Z J Surg 1984;54:29-35.

Smith GS, Barss P, Unintentional injuries in developing countries: The epidemiology of a neglected problem. Epidemiol Reviews 1991;13:228-61.

Smith GS, Langlois JA, Buechner JS. Methodological issues in using hospital discharge data to determine the incidence of hospitalised injuries. Am J Epidemiol 1991;134:1146-58.

Snow CA. It's not blank. St Martins-in-Deepdene: C. A. Snow and Associates, 1991.

Somerville CJ, McLean AJ. The cost of road accidents. Adelaide: The University of Adelaide Road Accident Research Unit, 1981.

Sommers RL. The Probability of Death Score: A measure of injury severity for use in planning and evaluating accident prevention. Accid Anal Prev 1983;15:259-66.

SPSS Inc. SPSS reference guide. Chicago: SPSS, 1990.

States JD, Viano DC. Injury Impairment and Disability Scales to assess the permanent consequences of trauma. Accid Anal Prev 1990;22:151-60. 
Statistics and Epidemiological Research Corporation. EGRET reference manual (revision 4) Seattle: SERC, 1989.

Steadman LA, Bryan RJ. Cost of road accidents in Australia. (occasional Paper 91) Canberra: Bureau of Transport and Communications Economics. 1988

Streiner DL, Norman GR. Health Measurement Scales. Oxford: Oxford University Press, 1989.

Suchman EA. A conceptual analysis of the accident phenomenon. In: Behavioural approaches to accident research. New York: Association for the Aid of Crippled Children, 1961;241-53.

Taylor RG. Assessment of childhood injury severity. Aust Paediatr J 1989;25:6-9.

Teijink JAW, Dwars BJ, Patka P, Haarman HJThM. Scoring multitrauma patients: which scoring system? Injury 1993;24:13-6.

Tepas JJ, Ramenofsky ML, Mollitt DL, Gans BM, DiScala C. The paediatric trauma score as a predictor of injury severity: an objective assessment. J Trauma 1988;28:4259.

The Euroqol Group. EuroQol - a new facility for the measurement of health-related quality of life. Health Policy 1990;16:199-208.

Thompson MS. Willingness to pay and accept risks to cure chronic disease. Am J Public Health 1986;76:392-6.

Torrance GW, Boyle MH, Horwood SP. Application of multiattribute utility theory to measure social preferences for health states. Oper Res 1982;30:1043-69.

Torrance GW. Utility approach to measuring health-related quality of life. J Chron Dis 1987;40:593-600.

Troy PN, Butlin NG. The cost of collisions. Adelaide: Cheshire, 1971.

Tsai SP, Bernacki EJ, Dowd CM. Incidence and cost of injury in an industrial population. J Occup Med 1989;31:781-4.

van Aalst JA, Morris JA, Yates HK, Miller RS, Bass SM. Severely injured geriatric patients return to independent living: A study of factors influencing function and independence. J Trauma 1991;31:1096-101.

Van Doorslaer E, Bouterr L. Assessing the economic burden of injuries due to accidents: methodological problems illustrated with some examples from the literature. Health Policy 1990;14:253-65.

Victorian Injury Surveillance System. Hazard. Melbourne: Monash University Accident Research Centre, 1994.

Vimpani G, Hartley P. Informational systems for the prevention of injury. Med J Aust 1989;150:470-472. 
Vimpani G. Injury Surveillance: a key to effective control of childhood injuries. Aust Paediatr J. 1989;25:10-3.

Vipulendran V, Mason AR, Sunderland R. Cost to the NHS of accidents to children in the West Midlands. BMJ 1988;296:611.

von Koch M, Berglund A, Nygren A, Tingvall C. Does a medical disabling injury influence health-related physiological and medical factors in the long run? Conference proceedings 2 nd World Conference on Injury Control, Atlanta, USA. May 1993:359.

Waller AE, Baker SP, Szocka A. Childhood injury deaths: National analysis and geographic variations. Am J Public Health 1989;79:310-5.

Waller J. Injury Control: A Guide to causes and prevention of Trauma. Lexington, Mass: D.C. Heath, 1985.

Waller J, Injury: Conceptual shifts and preventive implications. Ann Rev Public Health 1987;8:21-49.

Waller JA. Methodological issues in hospital-based Injury Research. J Trauma 1988;28:1632-6.

Waller JA, Payne SR, Skelly JM. Injuries to carpenters. J Occup Med 1989;31:687-92.

Waller JA, Payne SR, Skelly JM. Disability, direct cost, and payment issues in injuries involving woodworking and wood-related construction. Accid Anal Prev 1990;22:35160.

Waller JA. Injury Control in Perspective. Am J Public Health 1989;79:272-3.

Ward EGW, Bodiwala GG, Thomas PD. The importance of lower limb injuries in car crashes when cost and disability are considered. Accid Anal Prev 1992;24:613-20.

Wardrope J, Cross SF, Fothergill DJ. One year's experience of major trauma outcome study methodology. BMJ 1990;301:156-9.

Webb GR, Redman S, Wilkinson C, Sanson-Fisher RW. Filtering effects in reporting work injuries. Accid Anal Prev 1989;21:115-23.

Wesson DE, Williams JI, Spence LJ, Filler RM, Armstrong PF, Pearl RH. Functional outcome in paediatric trauma. J Trauma 1989;29:589-92.

Wheatley J, Cass D Traumatic deaths in children: the importance of prevention. Med J Aust 1989;150:72-8.

Wigglesworth EC Accidents in Australia: the need for research. Med $J$ Aust 1970;1:1113-20.

Wigglesworth EC, Fact or fable: towards the conceptualization of trauma. Med J Aust 1989;150:57-8.

Worksafe Australia. National Institute report: estimates of national occupational health and safety statistics 1991-1992 Canberra: AGPS, 1993. 
World Bank. Investing in Health. New York: Oxford University Press, 1993.

World Health Organization. Manual of the International Statistical Classification of Diseases, Injuries, and Causes of Death (ninth revision). Geneva: WHO, 1977.

World Health Organization. Constitution of the World Health Organization. Geneva: WHO, 1984.

World Health Organization. International classification of impairments, disabilities and handicaps. Geneva: WHO, 1980.

Wrightson P, Gronwell D. Time off work and symptoms after minor head injury. Injury 1981;12:445-54.

Yates DW, Heath DF, Mars E, Taylor RJ. A system for measuring the severity of temporary and permanent disability after injury. Accid Anal Prev 1991;23:323-9.

Zeidler F, Pletschen B, Scheunert D, Mattern B, Alt B, Miksch T, Eichendorf W, Reiss S. Development of a new injury cost scale. Accid Anal Prev 1993;25:675-87. 


\section{Appendix}

1 General practice questionnaire

2 Australian Capital Territory Injury Surveillance and Prevention (ACTISPP) questionnaire

3 Quality of Well-Being (QWB) scale scoring schedule

4 Health Consequences of Injury Questionnaire (HCIQ) version-1

5 Health Consequences of Injury Questionnaire ( $\mathrm{HCIQ}$ ) version-2

6 Australian Morbidity and Treatment Survey questionnaire 
Appendix-1

General practice questionnaire 


\section{ACT INJURY SURVEILLANCE AND PREVENTION PROJECT}

ACT BOARD OF HEALTH

I UNDERSTAND THAT THIS INFORMATION WILL BE USED FOR STATSTICAL PURPOSES ONLY AND THAT MY PRIVACY WILL BE MAINTANNED

PATIENT NAME:

ADDRESS:

SUBURB:

DATE OF BIRTH:

POSTCODE:

MALE $\square$ FEMALE

\begin{tabular}{|c|c|}
\hline WHEN? & $\begin{array}{l}\text { When did the injury oceur? } \\
\text { Date: } \\
\text { Time: } \\
\text { Is this your first visit to a doctor with this partlcular injury? Yes } \square \text { No } \square\end{array}$ \\
\hline WHERE? & $\begin{array}{l}\text { Where dld the Injury occur? e.g. at home in the tathrecm, at Coakin High School oval, on } \\
\text { Weston SL at NCSC maintenanca depl }\end{array}$ \\
\hline WHAT? & $\begin{array}{l}\text { What was the injured person doing at the time? e.g. washing hands, playing with friends, } \\
\text { driving a cas, moving pipes. } \\
\text { What went wrong to cause the injury? e.g.found pills, fell trem tree, cellided with car, dreffed } \\
\text { pice. }\end{array}$ \\
\hline HOW? & $\begin{array}{l}\text { How was the injury caused? e.g.swallowed Cigcxin pills, lancec on concrete, thrown agairs: } \\
\text { windscreen, pipe landed on foot }\end{array}$ \\
\hline SAFETY? & $\begin{array}{l}\text { What safety precautions were being used at the time? e.g. childproof borte cap. seat } \\
\text { berh safery shoes, bicycle heimet nil }\end{array}$ \\
\hline May we contact? & further Information is required? Yes $\square$ No $\square$ \\
\hline
\end{tabular}

THANK YOU FOR YOUR ASSISTANCE PLEASE HAND THIS FORM TO THE DOCTOR This pracice is paricipating in an Australia wide progran of injury and poisoning prevention.

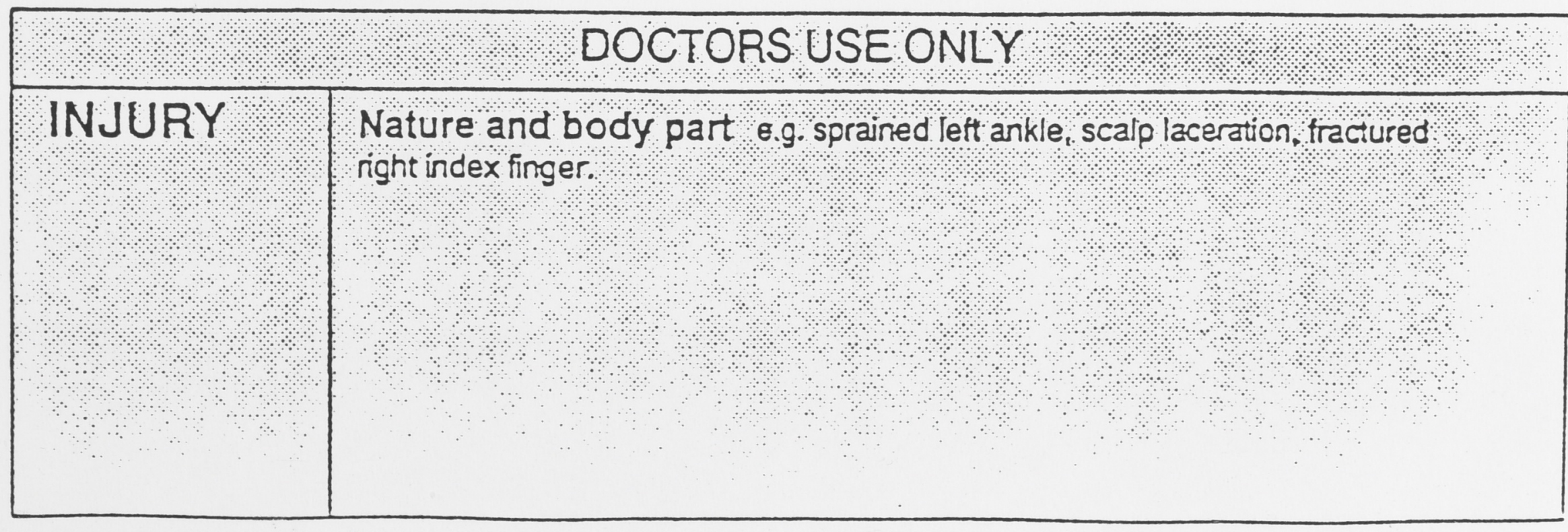


Appendix- 2

Australian Capital Territory Injury Surveillance and Prevention (ACTISPP) questionnaire 

privacy will be maintained

Patients Name

Address

Suburb

Date of Birth

Postcode

Male

Female

WHEN?

When did the injury occur?
Date

WHERE?

Where did the injury occur? (eg. at home in the bathroom, at Deakin High School oval, on Weston St, at ACTION Bus Maintenance depot)

WHAT?

What was the injured person doing at the time? (eg. washing hands, playing with friends, driving a car, moving pipes)

What went wrong to cause the injury? (eg found pills, fell from tree, collided with car, dropped pipe)

HOW?

How was the injury caused? (eg. swallowed Digoxin pills, landed on concrete, thrown against windscreen, pipe landed on foot)

What safety precautions were being used at the time? (eg. childproof bottle cap, seat belt, safety shoes, bicycle helmet, nil)

SAFETY? (1)

Was a specific product or article involved in the injury? Yes If Yes, please specify Brand Name or Type

No

If Yes, please specify Brand Name or Type
If a motor vehicle was involved please give details and indicate seat position
Make
Year
Type of Vehicle

Please show where you were sitting (eg. 1 - Driver)

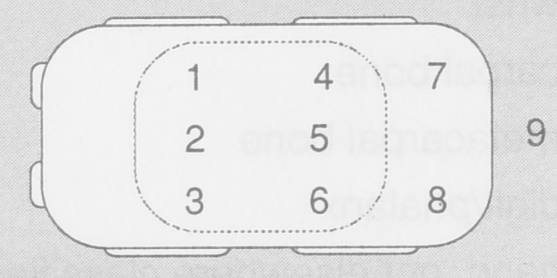

Did the injury occur at work? Yes

No

Will a worker's compensation claim be made? Yes

No

What is the occupation of the injured person?

In what business/industry is the injured person employed?

Is the injured person Aboriginal/Torres Strait Islander? Yes

No

What languages other than English are spoken in the injured person's home?

May we contact you if further information is required? Yes

No

Thank you for your assistance - Please hand this form to the Doctor

PA h2396 (6/92)

This hospital is participating in an Australia wide program of injury and poisoning prevention 


\section{Nature of Injury}

\begin{tabular}{lll}
\hline Soft Tissue & Select up to three items - Number most severe Injury as '1' \\
$\square$ cut/laceration & $\square$ burn, full thickness & Systemic and Special Injury \\
$\square$ Puncture & $\square$ burn, partial thickness & $\square$ ingestion \\
$\square$ bite & $\square$ damage to major blood vessel & $\square$ asphyxiation/respiratory difficulty \\
$\square$ superficial abrasion & $\square$ crushing injury & $\square$ electric shock \\
$\square$ penetrating wound & Bone/Tendon/Joint & $\square$ over-exertion, heat/cold stress. \\
$\square$ other wound, incl. amputation & $\square$ fracture & $\square$ concussion \\
$\square$ haematoma/bruising & $\square$ dislocation & $\square$ no injury detected \\
$\square$ haemorrhage & $\square$ sprain/strain &
\end{tabular}

\section{Body Part}

\section{Select up to three items - Number most severe Injury as ' 1 '}

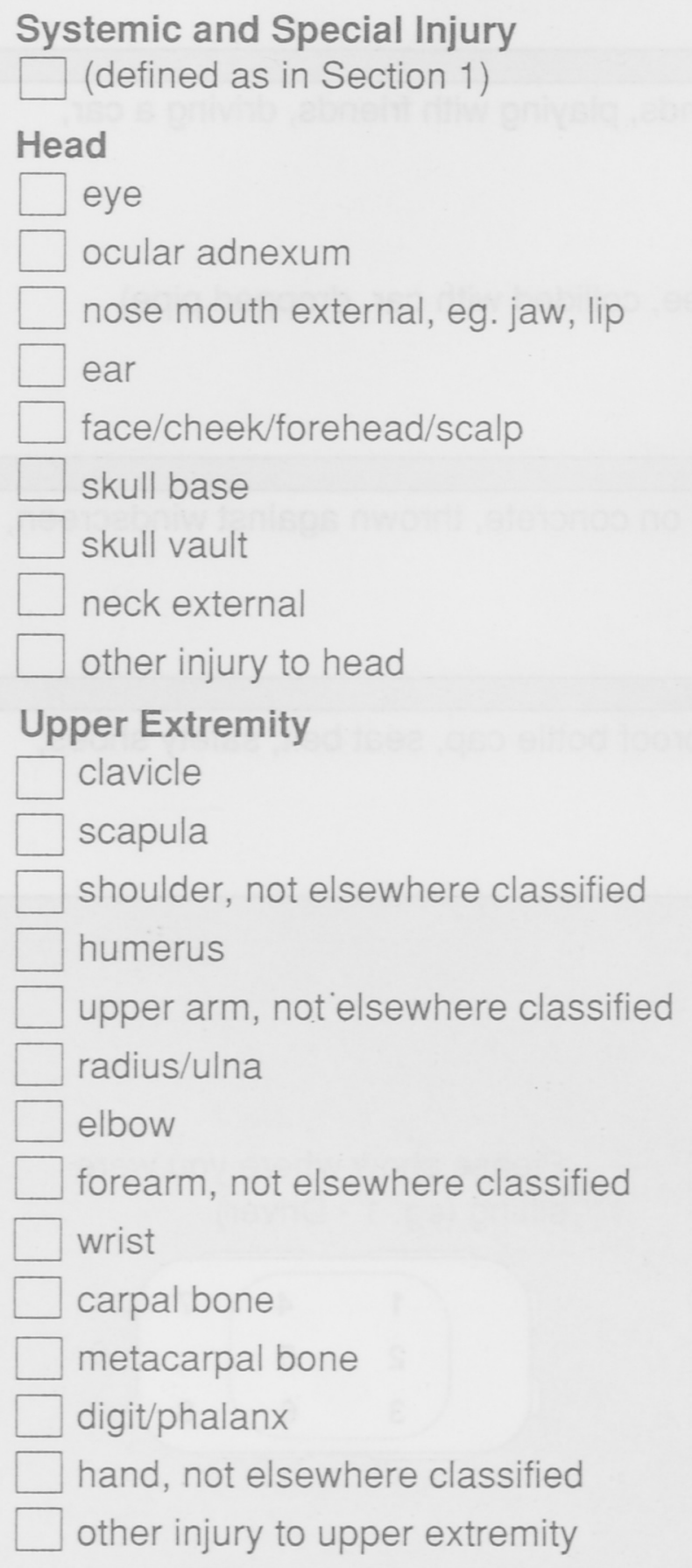

\section{Reported intent of Injury} Please tick appropriate box accidental injury self-inflicted, or possible so $\square$ alleged victim of assault, or possibly so unknown intent

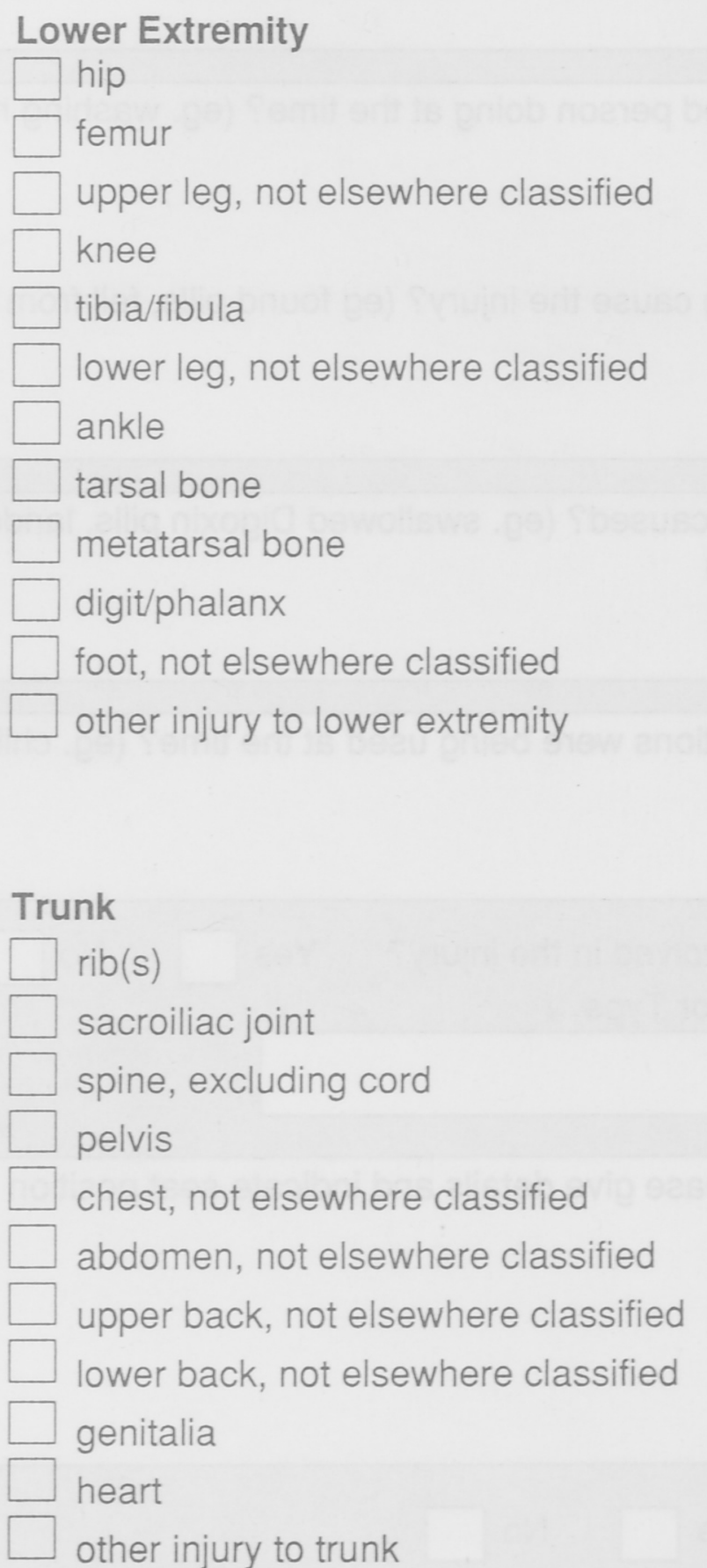

\section{Mode of Separation} Please tick appropriate box

$\square$ no treatment

treated, no referral

treated, A \& E review

treated, referred to outpt. dept.

treated, referred to family doctor

treated, other referral

admitted to hospital

transferred to other hospital

DOA

died in $A$ \& $E$

\begin{tabular}{l} 
Respiratory Tract \\
$\square$ pharynx \\
$\square$ larynx \\
$\square$ trachea \\
$\square$ bronchus \\
$\square$ lung \\
$\square$ other injury to respiratory tract \\
Digestive Tract \\
$\square$ mouth internal eg. gum, palate \\
$\square$ oesophagus \\
$\square$ stomach \\
$\square$ small bowel \\
$\square$ colon \\
$\square$ rectum \\
$\square$ liver \\
$\square$ spleen \\
$\square$ injury to other internal organ \\
$\square$ other injury to digestive tract \\
$\square$ thoracic spinal cord \\
$\square$ lumbar spinal cord \\
$\square$ prvous System \\
$\square$ brain, not concussion \\
$\square$ cervical spinal cord \\
$\square$ other injury to nervous system \\
\hline$\square$
\end{tabular}

\section{Severity Score}

For admissions only

$\square$ cardiorespiratory

$\square$ coma

$\square$ trauma

If transferred to another hopsital - please specify 
Appendix- 3

Quality of Well-Being (QWB) scale scoring schedule 
Table 1. Wse of Quallty of Well-being scale

symptom/Problem Complexes (CPX) Wlth Calculating Welghts

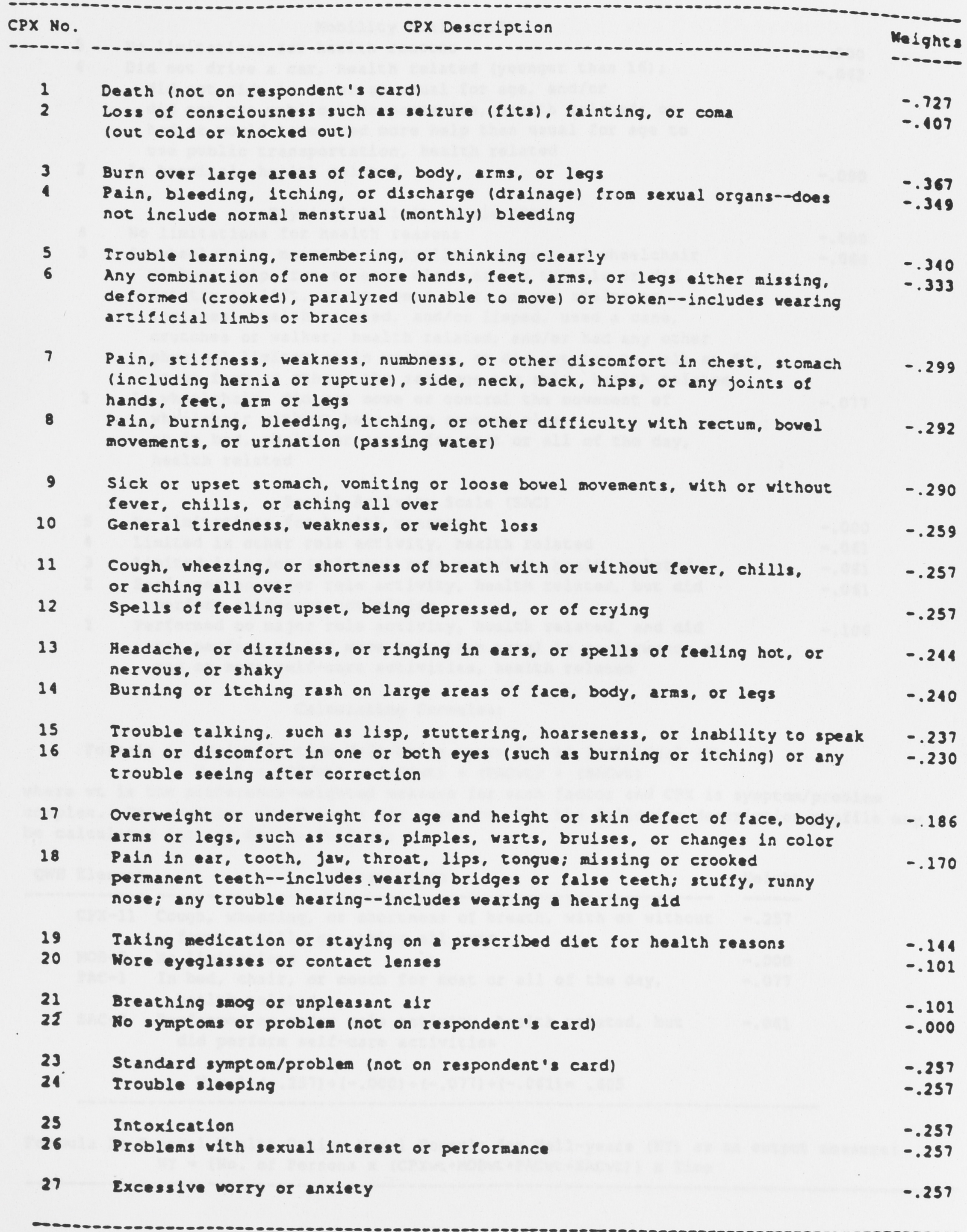


rable 2. Quabiey of Well-belng Scale Elements and Calcularlng Formulas

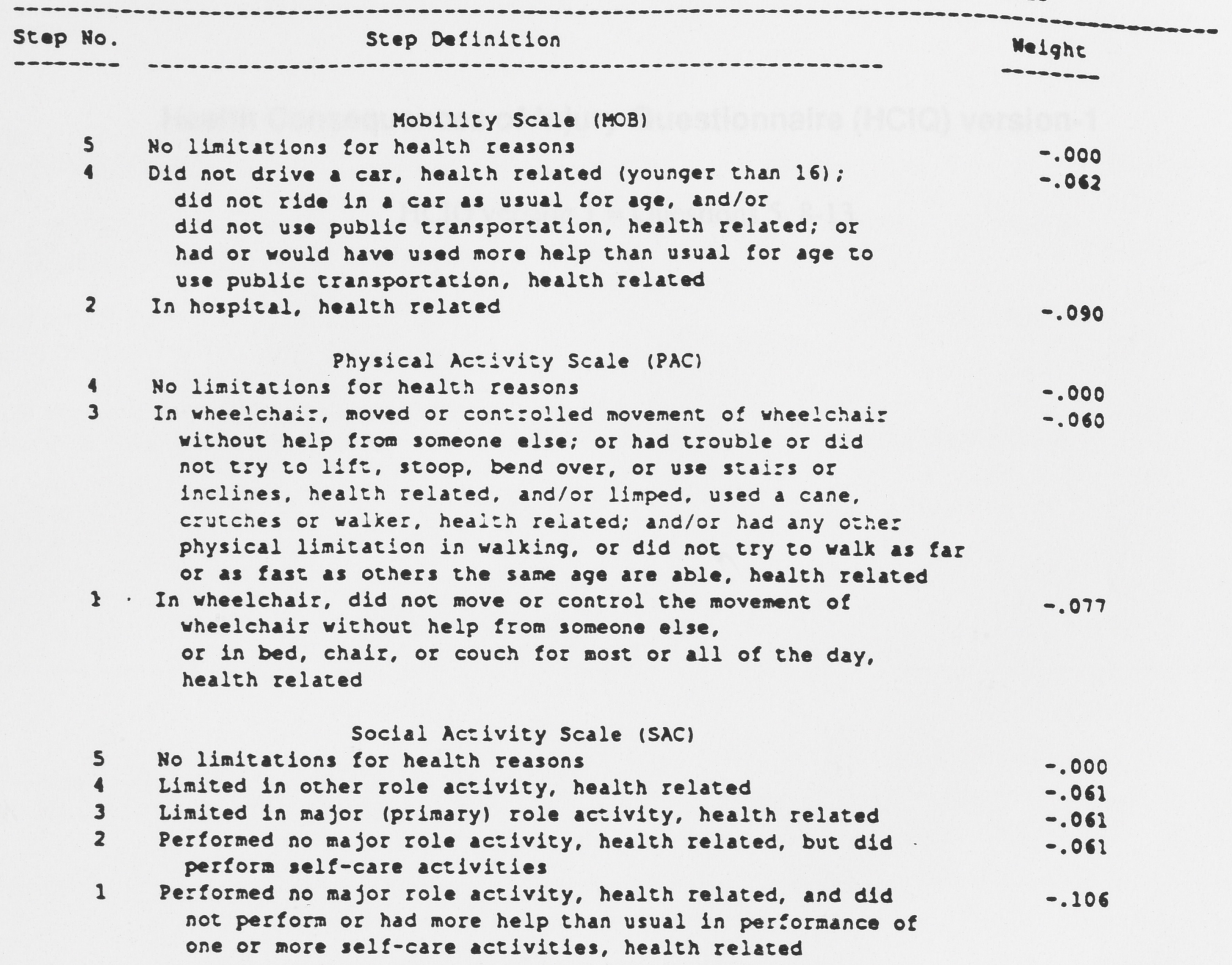

\section{Calculating Eormulas:}

Formula 1: Point-in-time Well-belng score for an Individual (W): $W=1+($ CPXWt + (MOBWT) + (PACWT) + (SACWT)

where $w$ is the preference-weighted measure for each factor and CPX 1s symptom/problem complex. For example, the $w$ score for a person with the following description proflle may be calculared for one day as follows:

\begin{tabular}{|c|c|c|}
\hline QWB Element & Description & Weight \\
\hline$C P X-11$ & $\begin{array}{l}\text { Cough, wheezing, or shortness of breath, with or without } \\
\text { fever, chill, or aching all over }\end{array}$ & -.257 \\
\hline HOB-5 & No dimleatlons & -.000 \\
\hline PAC-1 & $\begin{array}{l}\text { In bed, chalr, or couch for most or all of the day, } \\
\text { health related }\end{array}$ & -.077 \\
\hline SAC-2 & $\begin{array}{l}\text { Performed no major role ectivity, health related, but } \\
\text { did perform self-care activities } \\
\qquad-1+(-.257)+(-.000)+(-.077)+(-.061)=.605\end{array}$ & -.061 \\
\hline
\end{tabular}

Formula 2: General Bealeh Pollcy Model Formula for Well-years (WY) as an output aeasure:

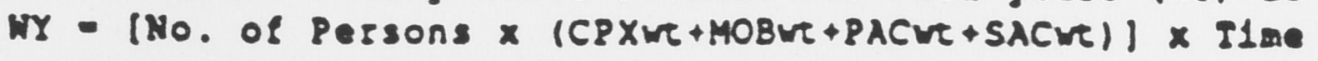


Appendix- 4

Health Consequences of Injury Questionnaire ( $\mathrm{HCIQ}$ ) version-1

HCIQ version 1 = Questions 5, 8-13. 
Date

1. In the week following your injury did you visit a doctor outside of hospital for treatment of your injury?

YES - NUMBER OF VISITS

NO

2. In the week following your injury did you visit the hospital for treatment of your injury?

YES

NUMBER OF VISITS

NUMBER OF VISITS

YES --

$X$-rays of your injury done outside hospital?

4. In the week following your injury did you go to any

of the following professionals for treatment of this injury?

NUMBER OF VISITS
a) a physiotherapist
b) a chiropractor
c) a dentist
d) a rehabilitation centre
e) another, please specify

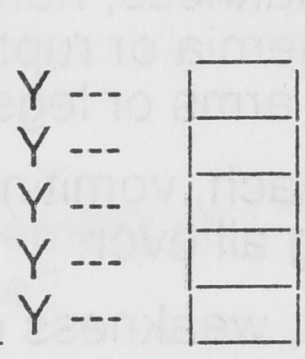

NO

NO

5. Have you had to stay in hospital for 24 hrs or more BECAUSE OF YOUR INJURY?

6. In the week since your injury occurred how much have you actually spent on the following aspects of your injury care? (Do not count bills you are expecting or have not yet paid)

medications

bandages and dressings

walking sticks crutches and other aids

home alterations necessary for coping with the injury

(Eg. adding ramps for a wheel chair)

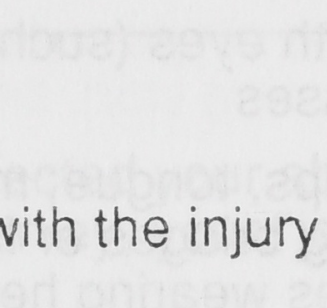

(Eg. taxi fares for physio. visits)

home help required because of your injury

OTHER (please specify, \& include lost earnings if appropriate)

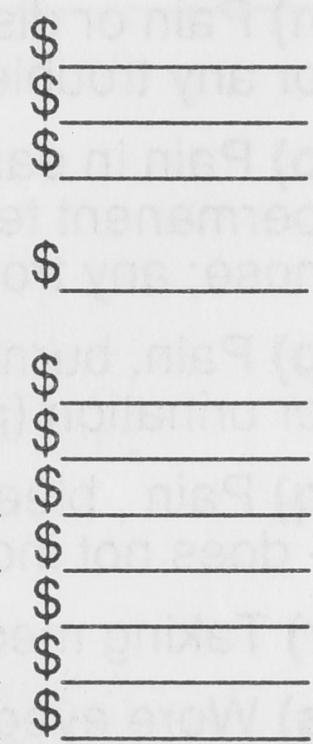

7. In your opinion, is your injury now completely better?

YES 
8. At the time of your injury, or in the week following, did you experience any of the following symptoms or problems BECAUSE OF THIS INJURY?

a) Loss of consciousness such as fainting, seizure (fits), or coma (out cold or knocked out)

b) Burn over large areas of face, body, arms or legs

c) Trouble learning, remembering, or thinking clearly

d) Any combination of one or more hands, feet, arms, or legs either missing, deformed (crooked), paralysed (unable to move) or broken- includes wearing artificial limbs or braces

e) Pain, stiffness, weakness, numbness, or other discomfort in chest, stomach (including hernia or rupture), side, neck, back, hips or any joints of hands, feet, arms or legs

f) Sick or upset stomach, vomiting or loose bowel movements, with or without fever, chills or aching all over.

g) General tiredness, weakness or weight loss

h) Cough, wheezing or shortness of breath with or without fever, chills or aching all over

i) Spells of feeling upset, being depressed, or of crying

j) Headache, or dizziness, or ringing in ears, or spells of feeling hot, or nervous or shaky

k) Burning or itching rash on large areas of face, body, arms or legs

I) Trouble talking, such as a lisp, stuttering, hoarseness, or inability to speak

m) Overweight or underweight for age and height or skin defect of face, body, arms or legs, such as scars, pimples, warts, bruises or changes in colour

n) Pain or discomfort in one or both eyes (such as burning or itching) or any trouble seeing despite glasses

o) Pain in ear, tooth, jaw, throat, lips, tongue; missing or crooked permanent teeth--includes wearing bridges or false teeth; stuffy, runny nose; any trouble hearing--includes wearing hearing aid

p) Pain, burning, itching, or other difficulty with rectum, bowel movements, or urination (passing water)

q) Pain, bleeding, itching, or discharge (drainage) from sexual organs - does not include normal menstrual (monthly) bleecing

r) Taking medication or staying on a prescribed diet for health reasons

s) Wore eyeglasses or contact lenses

t) Breathing smog or unpleasant air

u) Trouble sleeping

v) Intoxication

w) Problems with sexual interest or performance

x) Excessive worry or anxiety 
9. (i) At any time during the week following your injury, did your injury prevent you from

a) driving a car

YES NO

b) being a passenger in a car

YES NO

c) using public transport

YES NO

(ii) At any time during the week following your injury did your injury

cause you to need help to ride in a car or use public transport?

YES NO

10. (i) At any time during the week following your injury did you

spend the day in a bed, chair or couch BECAUSE OF YOUR INJURY?

YES NO

ii) At any time during the week following your injury did you

spend time in a wheelchair, BECAUSE OF YOUR INJURY?

YES NO

If YES, did you move or control the movement of

the chair without help from someone else?

YES NO

(iii) At any time during the week following your injury did your

injury prevent you from, or cause you to have difficulty with,

lifting, stooping, bending over or using stairs?

YES NO

(iv) At any time during the week following your injury did your

injury cause you to limp, use a stick, crutches or walker?

YES NO

(v) At any time during the week following your injury did your

injury prevent you from walking as far or as fast as is usual for you?

YES NO

11. Now we'd like to find out how your injury has affected your ability to work at your "job". For the purposes of this survey, your "job"

could be any of the following:-

a) full-time or part-time self employed or paid employment

b) houseduties

c) school, college or university studies

d) retired persons normal activities

e) preschooler's play activities

[NOTE: If you do not have a "job" go straight to question 12.]

(i) At any time during the week following your injury

has your injury COMPLETELY PREVENTED you from

being able to work at your "job"? NO

YES --- HOW MANY DAYS OFF?

(ii) At any time during the week following your injury

has your injury PARTIALLY RESTRICTED your

ability to work at your "job"?

NO YES --- ON HOW MANY DAYS? 
12. Here is a list of a wide range of activities people might engage in outside of their "job". Did your recent injury cause you to have more difficulty than usual with any of these activities at any time in the week following your injury?

a) Going shopping, handling persona! business, and so on.

b) Taking part in hobbies, games, play activities, and so on.

c) Visiting and meeting with friends, relatives and so on.

d) Taking part in church, or religious activities etc.

e) Taking part in community work, going to meetings and so on.

f) Attending movies, spectator sports, other entertainment and so on.

g) Looking for a job or other work.

h) Other activity similar to those above. Please specify

13. At any time in the week following your injury did you find you

i) did not DRESS because of your recent injury, or had help to DRESS (tying shoes, buttoning shirt, blouse, coat, etc)

ii) did not FEED SELF (did not eat, received fluids by vein etc) or had help to FEED SELF (being fed, having meat cut, bread buttered, etc) because of your injury

iii) did not use TOILET (Eg used a bedpan) because of your recent injury, or had help to use TOILET (getting on and off the seat, cleaning with tissues etc)

iv) did not TAKE A BATH or SHOWER because of your recent injury, or had help to TAKE A BATH/SHOWER, getting in or out of tub or shower, washing all parts of the body etc) 


\section{Appendix- 5}

Health Consequences of Injury Questionnaire ( $\mathrm{HCIQ}$ ) version-2

HCIQ version 2 = Questions 6, 8-13. 
1. In your opinion, is this injury now completely better?

YES

If YES, what was the date of full recovery? Thank you for your time, you need not answer the remaining questions.

NO If NO, please complete questions 2-13.

2. In the last week did you visit a doctor outside of hospital for treatment of this injury?

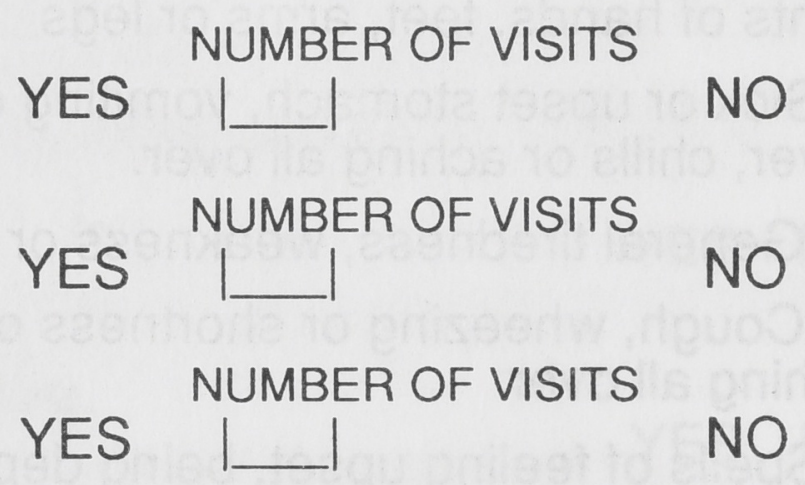

5. In the last week did you go to any of the following professionals for treatment of this injury?
a) a physiotherapist
b) a chiropractor
c) a dentist
d) a rehabilitation centre
e) another, please specify

YES NUMBER OF VISITS NO

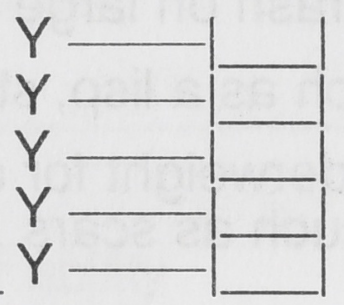

$\mathrm{N}$

$N$

$N$

$\mathrm{N}$

$\mathrm{N}$

6. In the last week have you had to stay in hospital for 24 hours or more BECAUSE OF THIS INJURY?

YES

NO

7. In the last week how much have you actually spent on the following aspects of your injury care?

medications

bandages and dressings

walking sticks crutches and other aids

home alterations necessary for coping with the injury

(Eg. adding ramps for a wheel chair)

transport costs

(Eg. taxi fares for physio. visits)

home help required because of your injury

OTHER (please specify)

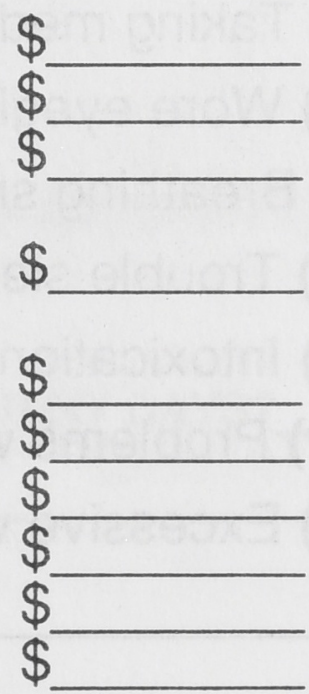


3. During the last week, did you experience any of the following symptoms or problems BECAUSE OF THIS INJURY?

a) Loss of consciousness such as fainting, seizure (fits), or coma (out cold or knocked out)

b) Burn over large areas of face, body, arms or legs

c) Trouble learning, remembering, or thinking clearly

d) Any combination of one or more hands, feet, arms, or legs either missing, deformed (crooked), paralysed (unable to move) or broken- includes wearing artificial limbs or braces

e) Pain, stiffness, weakness, numbness, or other discomfort in chest, stomach (including hernia or rupture), side, neck, back, hips or any joints of hands, feet, arms or legs

f) Sick or upset stomach, vomiting or loose bowel movements, with or without fever, chills or aching all over.

g) General tiredness, weakness or weight loss

h) Cough, wheezing or shortness of breath with or without fever, chills or aching all over

i) Spells of feeling upset, being depressed, or of crying

j) Headache, or dizziness, or ringing in ears, or spells of feeling hot, or nervous or shaky

k) Burning or itching rash on large areas of face, body, arms or legs

I) Trouble talking, such as a lisp, stuttering, hoarseness, or inability to speak

m) Overweight or underweight for age and height or skin defect of face, body, arms or legs, such as scars, pimples, warts, bruises or changes in colour

n) Pain or discomfort in one or both eyes (such as burning or itching) or any trouble seeing despite glasses

o) Pain in ear, tooth, jaw, throat, lips, tongue; missing or crooked permanent teeth--includes wearing bridges or false teeth; stuffy, runny nose; any trouble hearing--includes wearing hearing aid

p) Pain, burning, itching, or other difficulty with rectum, bowel movements, or urination (passing water)

q) Pain , bleeding, itching, or discharge (drainage) from sexual organs

- does not include normal menstrual (monthly) bleeding

r) Taking medication or staying on a prescribed diet for health reasons

s) Wore eyeglasses or contact lenses

t) Breathing smog or unpleasant air

u) Trouble sleeping

v) Intoxication

w) Problems with sexual interest or performance

x) Excessive worry or anxiety 
9. (i) At any time during the last week, did your injury prevent you from
a) driving a car
b) being a passenger in a car
c) using public transport
(ii) At any time during the last week did your injury cause you
to need help to ride in a car or use public transport?

$\begin{array}{ll}\text { YES } & \text { NO } \\ Y & N \\ Y & N \\ Y & N \\ Y & N\end{array}$

10. (i) At any time during the last week did you spend the day in a bed, chair or couch BECAUSE OF THIS INJURY?

ii) At any time during the last week did you spend time in a wheelchair, BECAUSE OF THIS INJURY?

Did you move or control the movement of the chair without help from someone else?

(iii) At any time during the last week did your injury prevent you from, or cause you to have difficulty with, lifting, stooping, bending over or using stairs?

(iv) At any time during the last week did your injury cause you to limp, use a stick, crutches or walker?

(v) At any time during the last week did your injury prevent you from walking as far or as fast as is usual for you?

11. Now we'd like to find out how your injury has affected your ability to work at your "job". For the purposes of this survey, your "job" could be any of the following:-
a) full-time or part-time self employed or paid employment
b) houseduties
c) school, college or university studies
d) retired persons normal activities
e) preschooler's play activities

[NOTE: If you do not have a "job" go straight to question 12.]

(i) At any time during the last week has your injury COMPLETELY PREVENTED you from being able to work at your "job"?

NO YES - HOW MANY DAYS OFF?

(ii) At any time during the last week has your injury PARTIALLY RESTRICTED your ability to work at your "job"?

NO YES - ON HOW MANY DAYS? 
12. Here is a list of a wide range of activities people might engage in outside of their "job". Did your recent injury cause you to have more difficulty than usual with any of these activities at any time during the last week?

a) Going shopping, handling personal business, and so on.

b) Taking part in hobbies, games, play activities, and so on.

c) Visiting and meeting with friends, relatives and so on.

d) Taking part in church, or religious activities etc.

e) Taking part in community work, going to meetings and so on.

f) Attending movies, spectator sports, other entertainment and so on.

g) Looking for a job or other work.

h) Other activity similar to those above. Please specify

13. During the last week did you find you

i) did not DRESS because of your recent injury, or had help to DRESS (tying shoes, buttoning shirt, blouse, coat, etc)

ii) did not FEED SELF (did not eat, received fluids by vein etc) or had help to FEED SELF (being fed, having meat cut, bread buttered, etc) because of your injury

iii) did not use TOILET (Eg used a bedpan) because of your recent injury, or had help to use TOILET (getting on and off the seat, cleaning with tissues etc)

iv) did not TAKE A BATH or SHOWER because of your recent injury, or had help to TAKE A BATH/SHOWER, getting in or out of tub or shower, washing all parts of the body etc) 


\section{Appendix- 6}

Australian Morbidity and Treatment Survey questionnaire 


\section{Appendix 2: Encounter recording form}

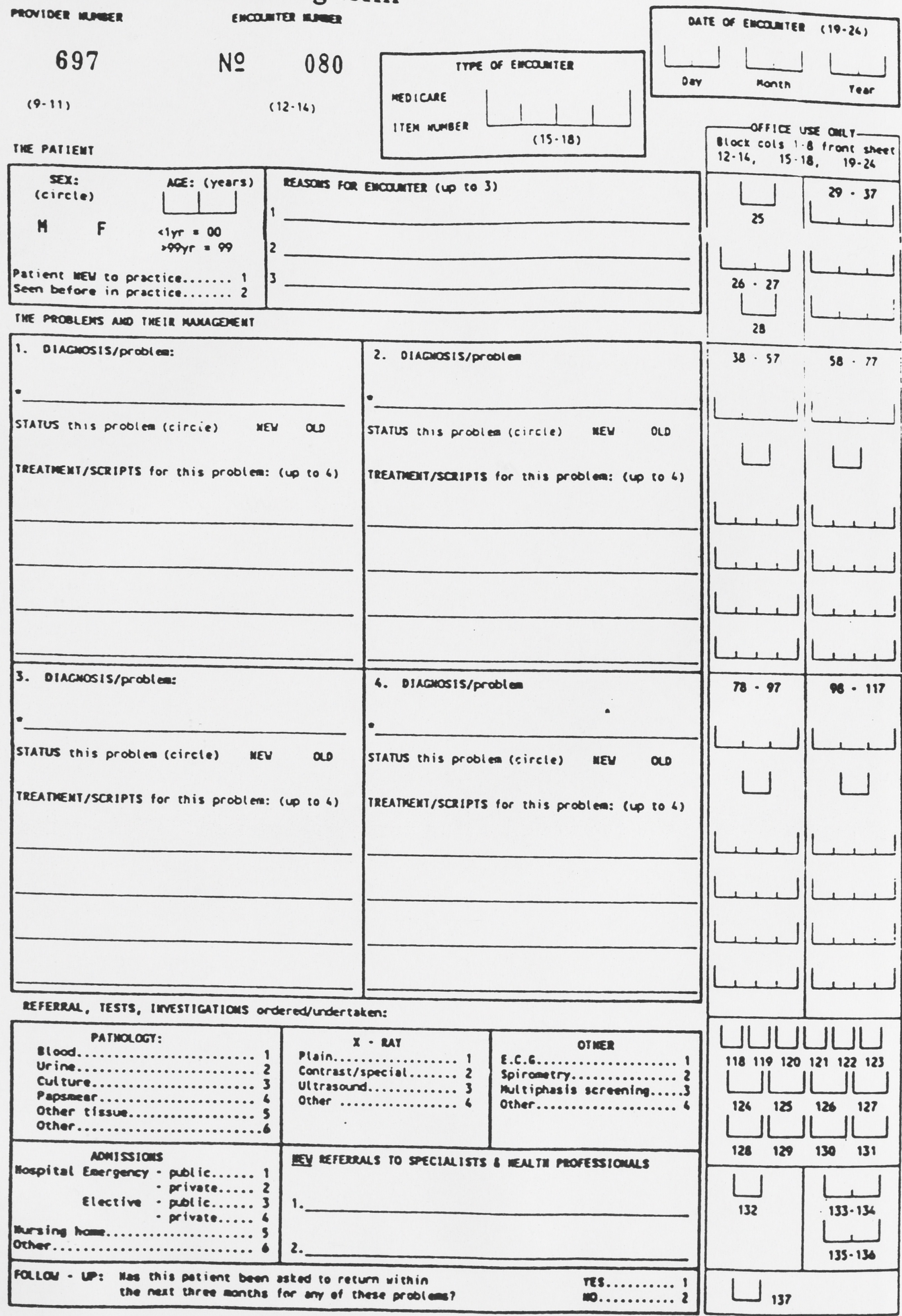

